\title{
MSTAR2019 Code Description and User's Manual
}

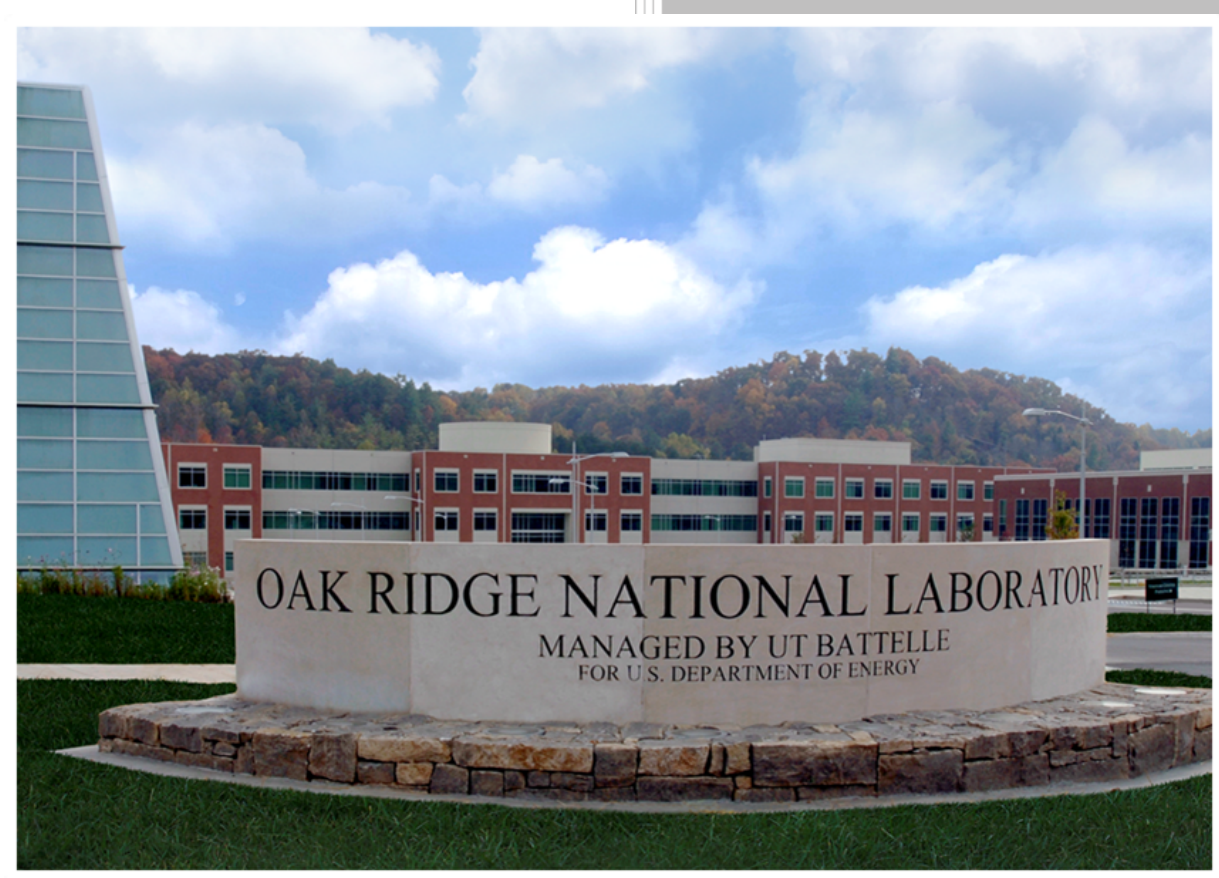

\section{Approved for public release.} Distribution is unlimited.

Charles F. Weber Israel J. Huff Jordan P. Lefebvre

April 2020 


\section{DOCUMENT AVAILABILITY}

Reports produced after January 1, 1996, are generally available free via US Department of Energy (DOE) SciTech Connect.

Website www.osti.gov

Reports produced before January 1, 1996, may be purchased by members of the public from the following source:

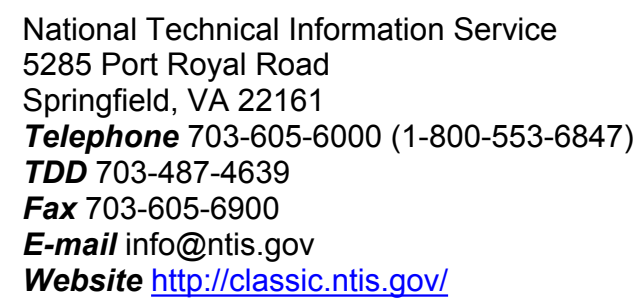

Reports are available to DOE employees, DOE contractors, Energy Technology Data Exchange representatives, and International Nuclear Information System representatives from the following source:

Office of Scientific and Technical Information

PO Box 62

Oak Ridge, TN 37831

Telephone 865-576-8401

Fax 865-576-5728

E-mail reports@osti.gov

Website http://www.osti.gov/contact.html

This report was prepared as an account of work sponsored by an agency of the United States Government. Neither the United States Government nor any agency thereof, nor any of their employees, makes any warranty, express or implied, or assumes any legal liability or responsibility for the accuracy, completeness, or usefulness of any information, apparatus, product, or process disclosed, or represents that its use would not infringe privately owned rights. Reference herein to any specific commercial product, process, or service by trade name, trademark, manufacturer, or otherwise, does not necessarily constitute or imply its endorsement, recommendation, or favoring by the United States Government or any agency thereof. The views and opinions of authors expressed herein do not necessarily state or reflect those of the United States Government or any agency thereof. 
Nuclear Nonproliferation Division

\title{
MSTAR2019 CODE DESCRIPTION AND USER'S MANUAL
}

\author{
Charles F. Weber \\ Israel J. Huff \\ Jordan P. Lefebvre
}

April 2020

Prepared by

OAK RIDGE NATIONAL LABORATORY

Oak Ridge, TN 37831-6283

managed by

UT-BATTELLE, LLC

for the

US DEPARTMENT OF ENERGY

under contract DE-AC05-00OR22725 



\section{CONTENTS}

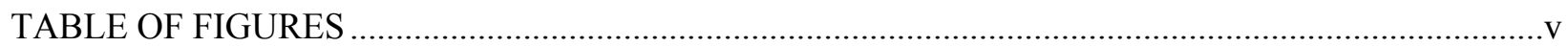

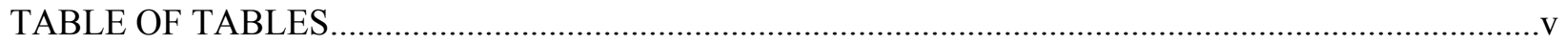

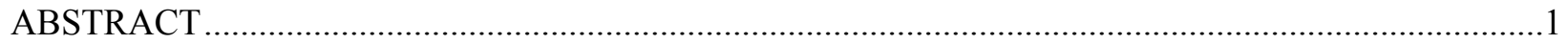

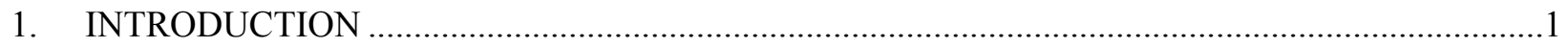

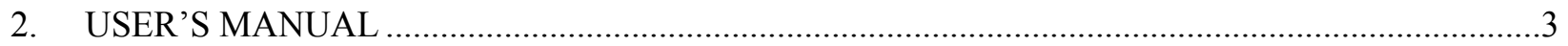

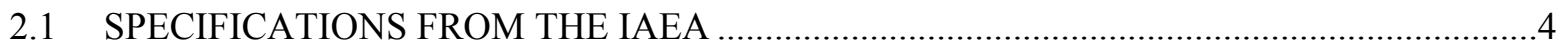

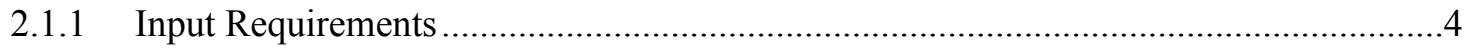

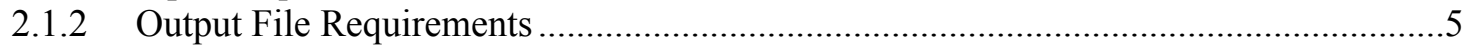

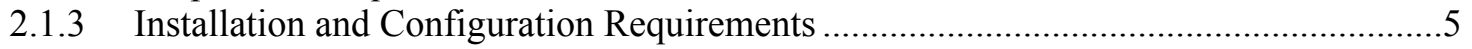

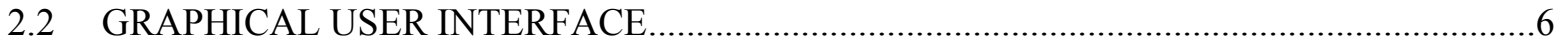

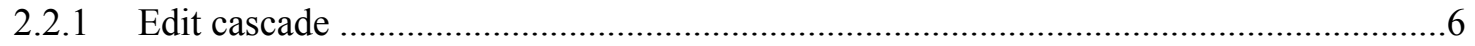

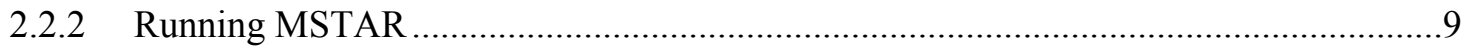

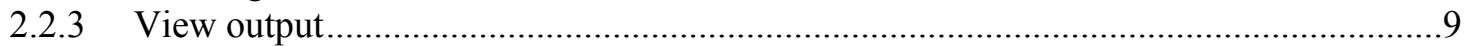

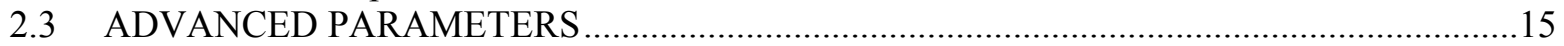

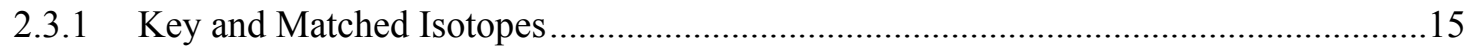

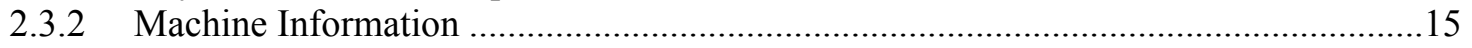

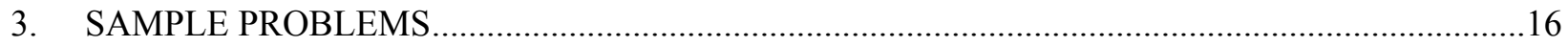

3.1 STANDARD PRODUCTION OF LOW ENRICHED URANIUM ....................................16

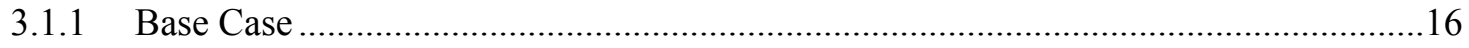

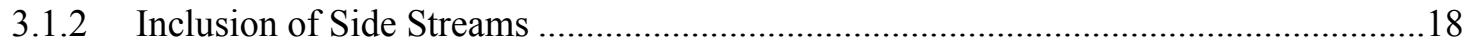

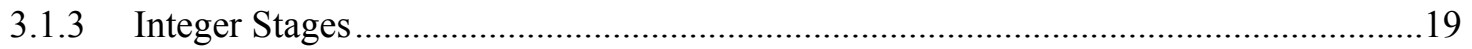

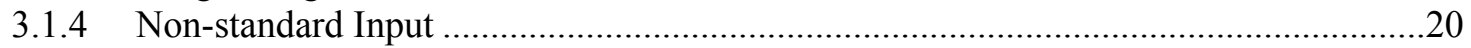

3.2 PRODUCTION OF HIGHLY ENRICHED URANIUM FROM RECYCLED

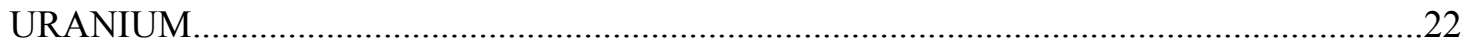

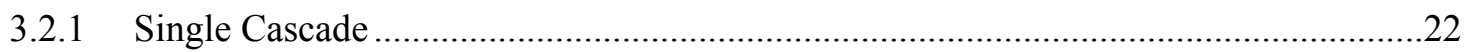

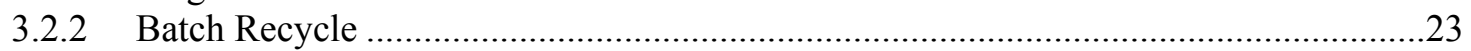

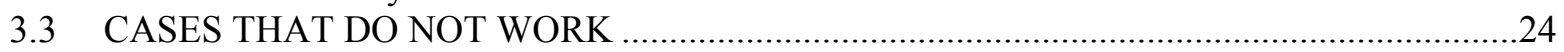

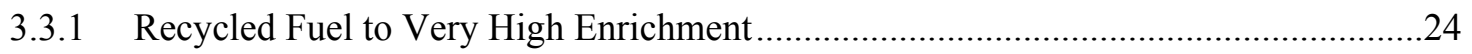

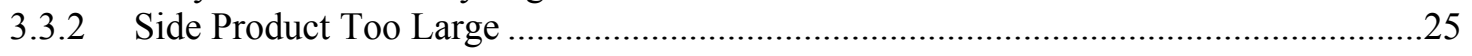

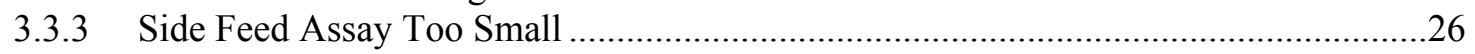

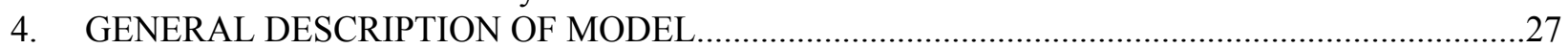

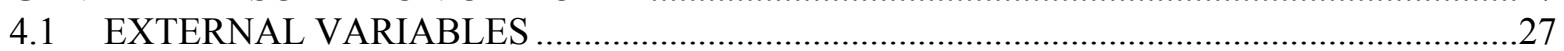

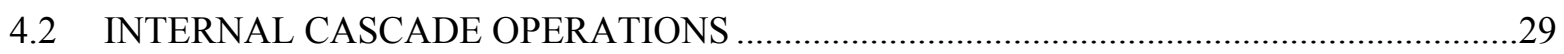

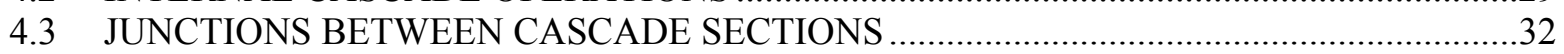

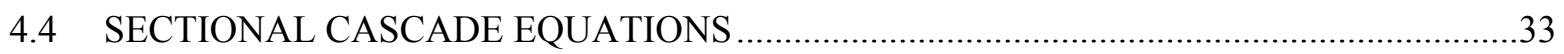

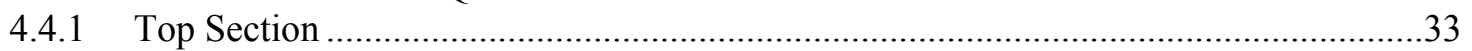

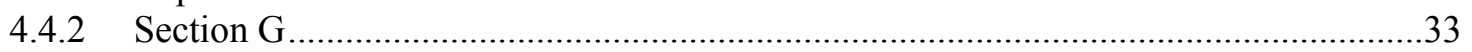

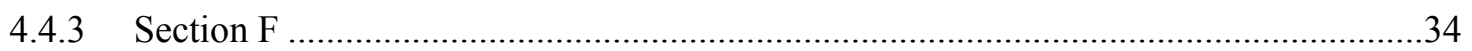

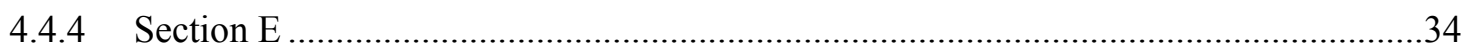

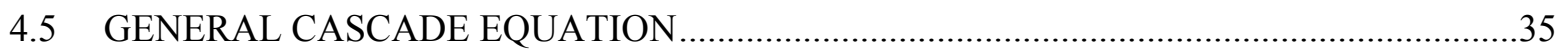

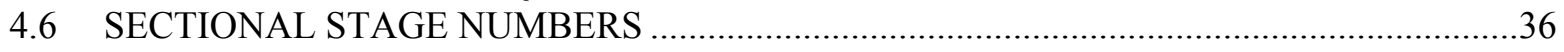

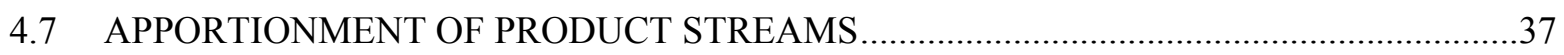

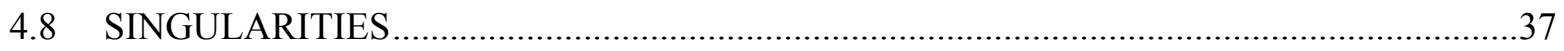

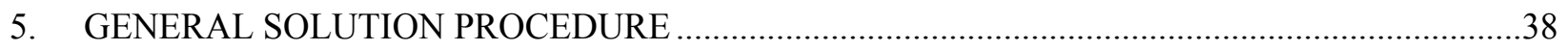

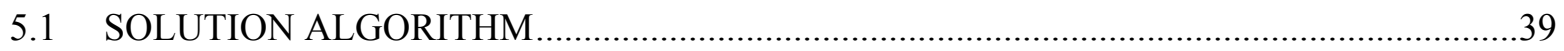

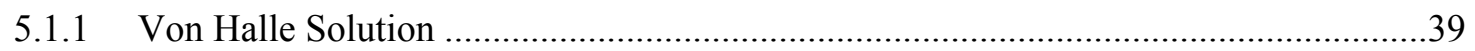

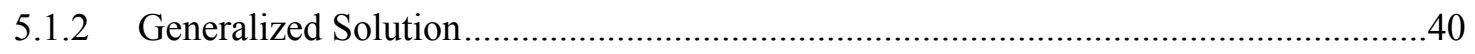

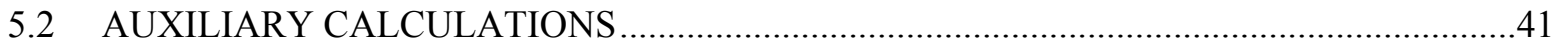




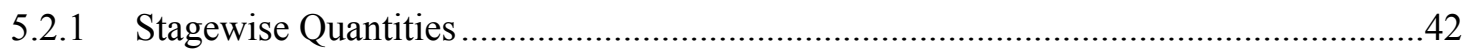

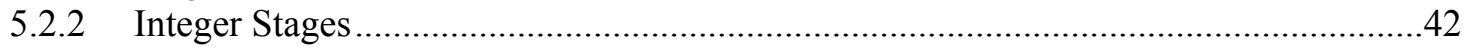

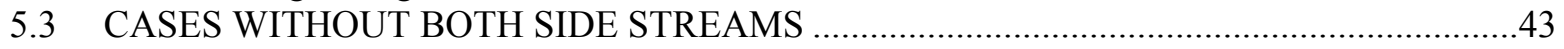

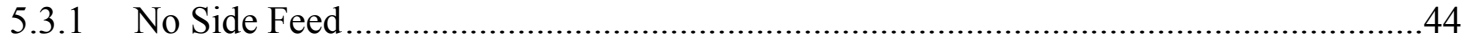

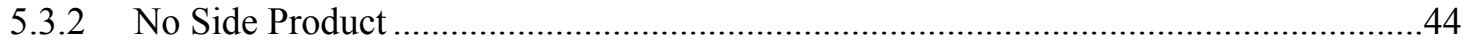

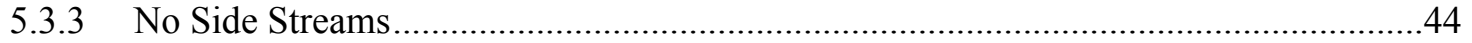

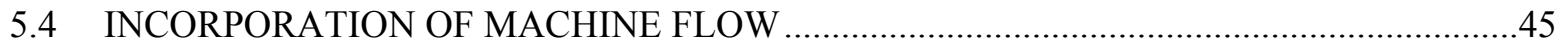

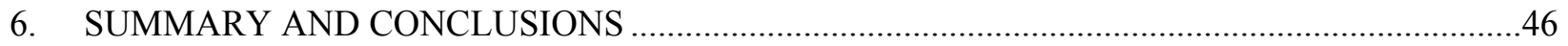

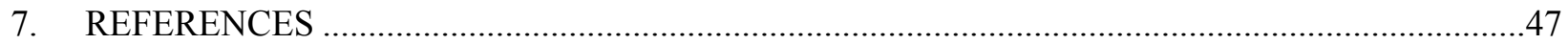

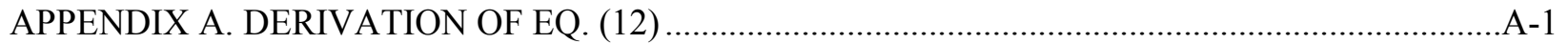

APPENDIX B. VON HALLE TREATMENT OF SECTION JUNCTION POINTS ...............................

APPENDIX C. DEPENDENCES OF GOVERNING EQUATIONS …...............................................

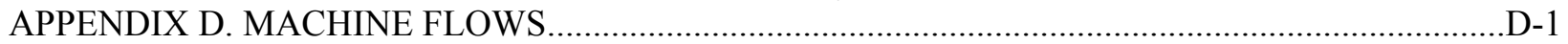

APPENDIX E. SUMMARY OF MODELING EQUATIONS ........................................................

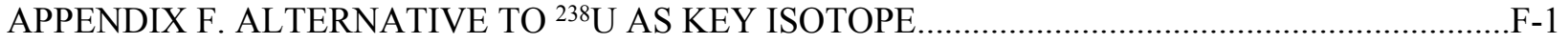

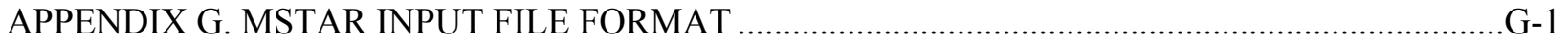




\section{TABLE OF FIGURES}

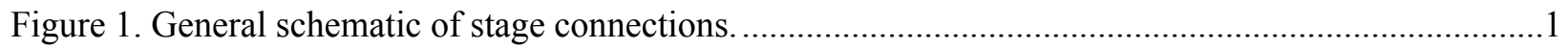

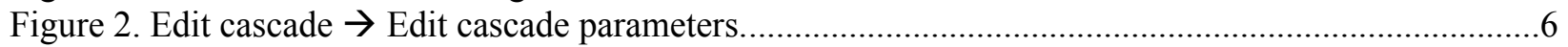

Figure 3. Edit cascade $\rightarrow$ Edit cascade parameters. Side feed checked. ...............................................

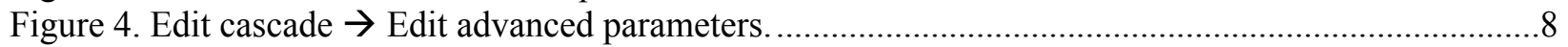

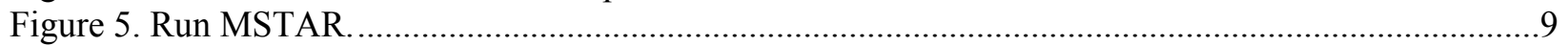

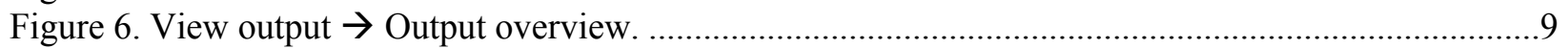

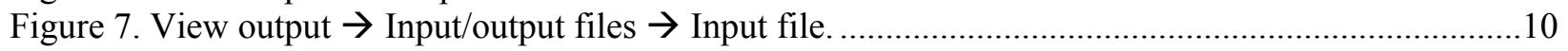

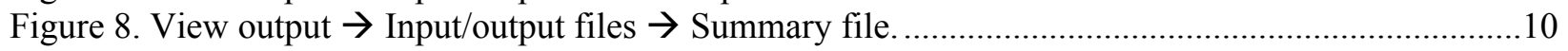

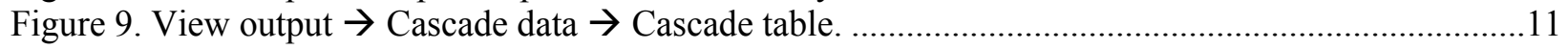

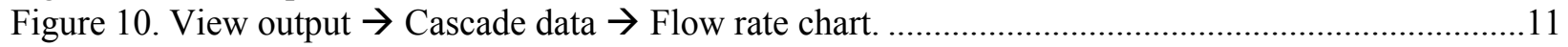

Figure 11. View output $\rightarrow$ Cascade data $\rightarrow$ Machines chart. Machines not set......................................12

Figure 12. View output $\rightarrow$ Cascade data $\rightarrow$ Machines chart. Machines set. ............................................12

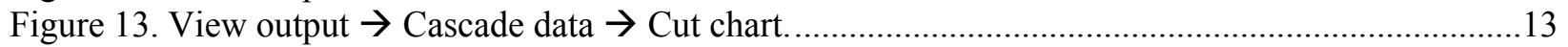

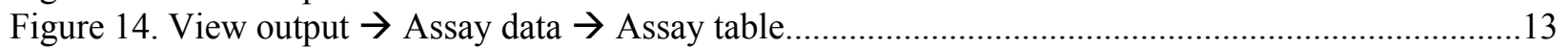

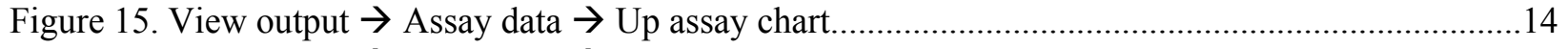

Figure 16. View output $\rightarrow$ Assay data $\rightarrow$ Up assay correlation chart. ...................................................14

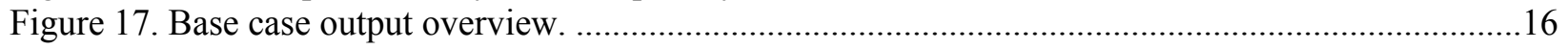

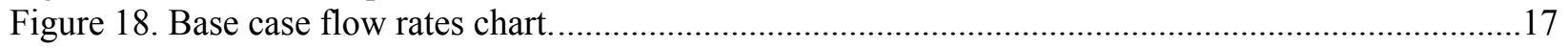

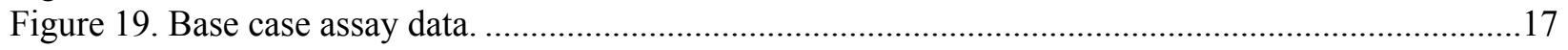

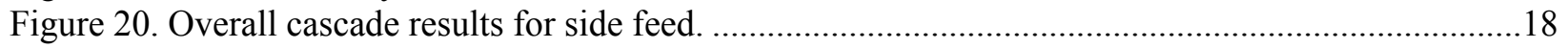

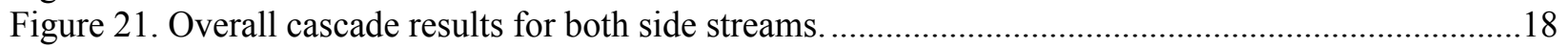

Figure 22. Output overview for side streams and integer stages. .......................................................19

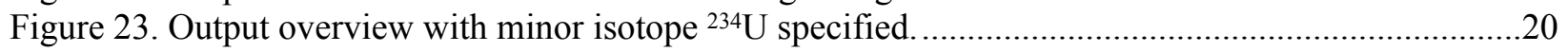

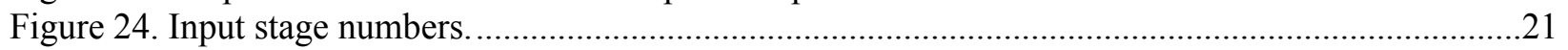

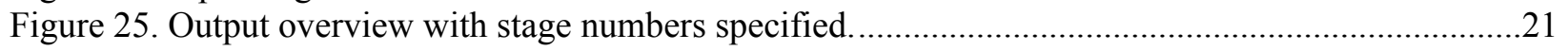

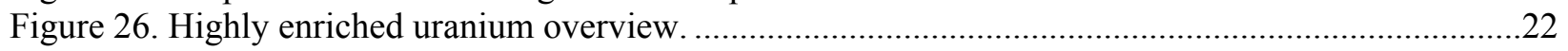

Figure 27. Machine chart for producing highly enriched uranium. .............................................22

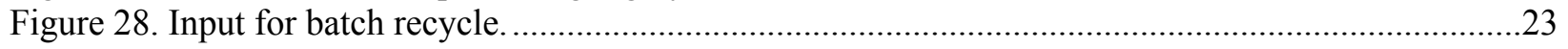

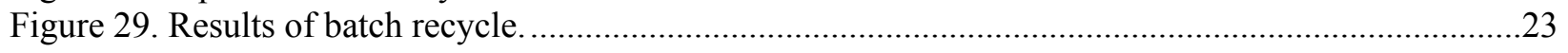

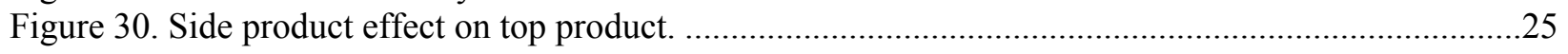

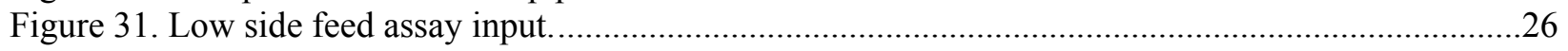

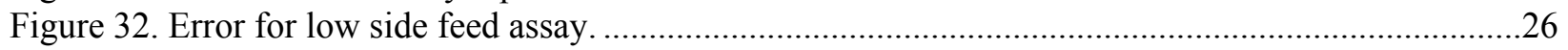

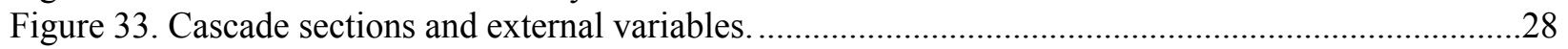

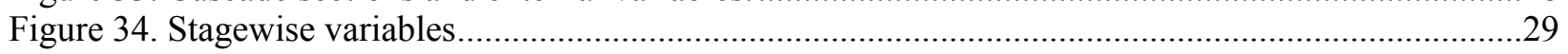

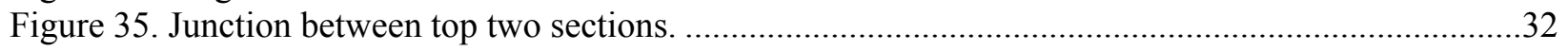

\section{TABLE OF TABLES}

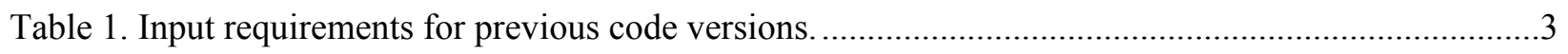

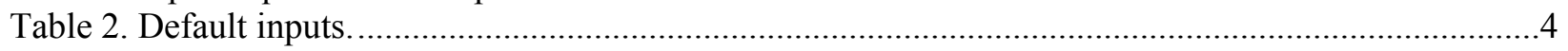

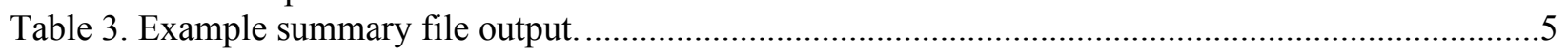

Table 4. Batch recycle to obtain highly enriched uranium from reprocessed uranium. ..............................24

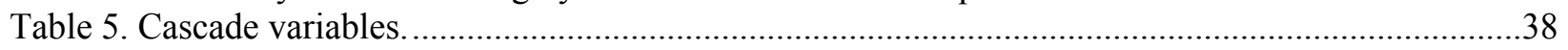

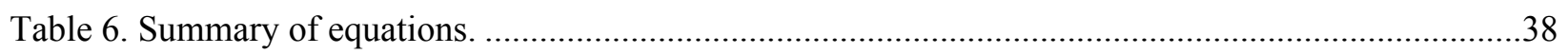

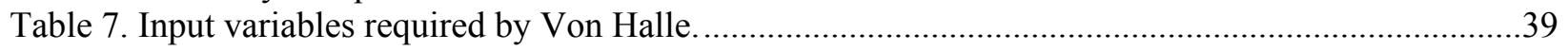

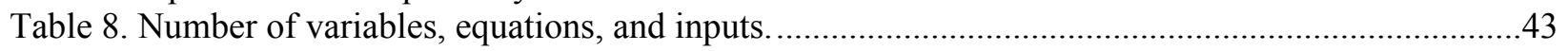





\begin{abstract}
This report describes an ideal cascade model of uranium enrichment that includes side feed and side product streams and flexible input options. It is based on the original MSTAR model developed by Ed Von Halle and implemented in a Visual Basic code. The current version allows the user to specify integer numbers of stages or the stage numbers of external flows instead of assays in those flows. The computational engine is written in FORTRAN-90 and is invoked by a user-friendly GUI written in C++. This version of MSTAR has been demonstrated to operate on Linux, Mac, and Windows platforms, and has undergone significant testing and quality analysis. A number of examples are presented to illustrate the operation of the code and guide the user. The code is extremely fast, and results are returned immediately. The input and output have been specially configured for analysis by the environmental sampling team of the International Atomic Energy Agency (IAEA). A number of additional output features are included to assist the user in visualizing computational results and downloading data to files for use in other analysis software.
\end{abstract}

\title{
1. INTRODUCTION
}

There are a number of facilities around the world that are engaged in the process of enriching natural uranium. The facilities in countries that have signed international safeguard agreements with the IAEA are monitored via an assortment of technical measures, including on-site inspections, nondestructive survey tools, material balance evaluations, design information verification, as well as environmental sampling. These procedures are designed to assure the material is being used for its intended and declared purpose and not for proliferation activities. The use of computational toolsets allows for the confirmation of declared activities in many of these areas, particularly in environmental sampling where measurements of small samples in or near a facility can be used along with model predictions to verify declared activities at the site.

Shown in Figure 1 is a simplified schematic of connections between stages in an enrichment facility. Cascade models do not involve detailed machine performance but assume basic attributes of machine operations that are obtained from testing data or from simulations of machine performance. They assume that a number of identical machines operate in parallel to make up operational stages and that different stages are connected in series. The more machines there are in a stage, the greater throughput can be achieved. Each stage increases the product enrichment, so more stages result in higher overall enrichment.

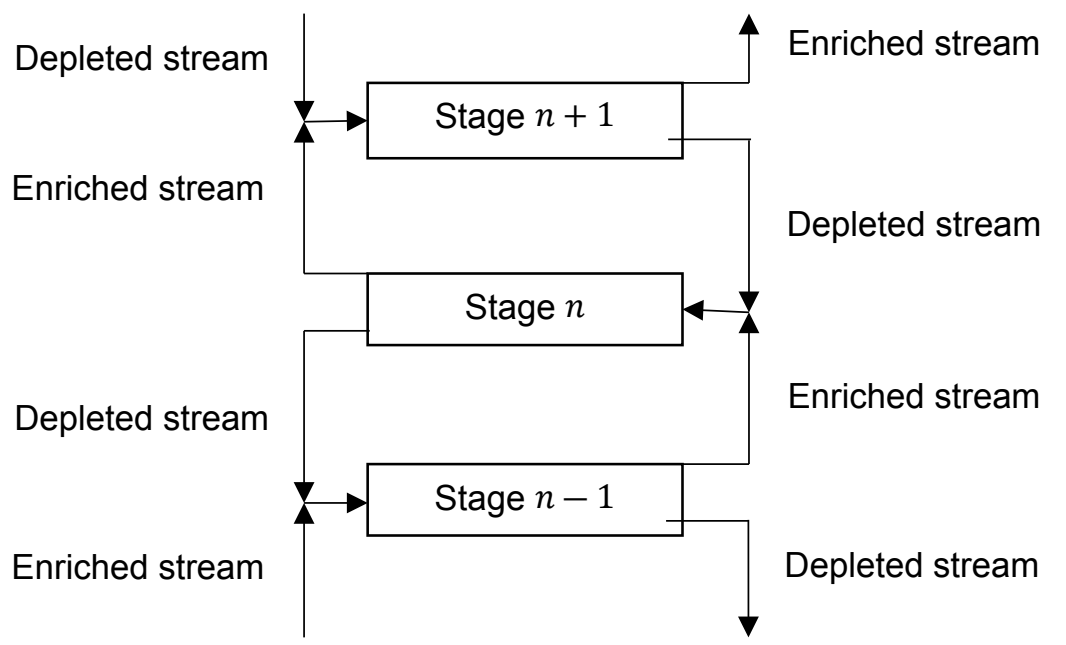

Figure 1. General schematic of stage connections. 
The concept of an ideal cascade presents both a simple and an optimized description of cascade performance for separation of ${ }^{235} \mathrm{U}$ from ${ }^{238} \mathrm{U}$. The ideal cascade assumes that no mixing losses occur when a depleted stream from a higher stage is combined with an enriched stream from a lower stage (see Figure 1); hence, it constitutes the most efficient possible operation. It also simplifies calculations because the assumption of equality for these two streams reduces the number of variables that must be calculated. When more than two isotopes are considered, the assumptions of the ideal cascade result in a mixedabundance-ratio cascade (MARC), in which mixing losses can be eliminated for only one isotope. This isotope is termed the "matched" isotope and is almost always ${ }^{235} \mathrm{U}$. The additional isotopes create more complication in modeling, but MARC still represents a simplification of the enrichment processes. Both the ideal cascade and MARC also assume that the separation performance at each stage is the same (i.e., the separation factor is constant for all stages of the cascade).

During the early days of cascade modeling, the focus of most modeling research was on gaseous diffusion, in which isotopic content at each stage was not very different from adjacent stages. Thus, cascade models were well approximated by differential equations $[1,2,3]$. With the advent of more advanced gas centrifuge designs, the isotopic content of adjacent stages was considerably different. The differential assumptions were no longer valid, and new modeling approaches were required. Primary efforts in this area were led by Ed Von Halle, who derived algebraic equations for cascades of machines with large separation factors [4]. He did additional development to include side feed and side product streams into his MARC model [5]. This work was innovative and elegant but apparently was never published or presented in an open forum. However, it formed the basis for the original MSTAR code [6], written in Visual Basic and distributed to the IAEA in 1996 as a deliverable of the U.S. Support Program. The code calculates the number of stages, stagewise flow rates, and isotopic assays for a given enrichment operation. It is based on standard mass balances, as is common in chemical process modeling (i.e., isotopic mass and flow balances are taken over different control volumes in the system). The code imposes strict input requirements, at least in part to provide numerical stability for the solution algorithm, and initially was limited to separation of uranium isotopes ${ }^{232} \mathrm{U},{ }^{234} \mathrm{U},{ }^{235} \mathrm{U},{ }^{236} \mathrm{U}$, and ${ }^{238} \mathrm{U}$.

Von Halle's modeling assumes the following relationship between separation factors for different isotopes:

$$
\ln \left(\alpha_{i}\right)=\left[\frac{M_{k}-M_{i}}{M_{k}-M_{m}}\right] \ln (\alpha), \quad i=1,2, \ldots, I
$$

where

$M_{i}=$ atomic weight of isotope $i$,

$m=$ index of the isotope whose abundance is matched in the cascade (usually ${ }^{235} \mathrm{U}$ ),

$k=$ index of the key component (usually ${ }^{238} \mathrm{U}$ ), the isotope against which all others are compared.

It is easy to see from Eq. (1) that the quoted separation factor $\alpha$ is identical to the separation factor for the matched component $\alpha_{m}$. In the past, this model has been applied to any type of enrichment process in which separation is based on mass difference of isotopes, such as gas centrifuge, gaseous diffusion, and vortex tube. This usage was justified in an early report by Blumkin and Von Halle [7], which was part of a series known as the MIST reports (Minor Isotope Data as a Safeguards Technique). These authors concluded that for an ideal cascade, the minor isotope separation factor is related to that of ${ }^{235} \mathrm{U}$ by Eq. (1) for both gaseous diffusion and gas centrifuge separations. The appropriateness of Eq. (1) for such diverse and generalized applications was questioned by Wood [8], who derived different separation factors for 
gas centrifuge and gaseous diffusion and noted very small differences in some cascade calculations. He proposed alternatives to Eq. (1) that were included as options in the earlier version of MSTAR $[9,10]$. However, the validity of Eq. (1) was also established by earlier references using only two components [11,12] and Harink-Snijders [13], who does so for three components. Because Eq. (1) has been widely accepted and experimentally verified, it alone is included in this work.

In 2012, an update to the MSTAR code was commissioned by the IAEA [9], which relaxed some of the strict input requirements and provided a code that would operate on a number of operating systems. The input flexibility was described in an open publication [10], and limitations to the basic cascade model were noted therein. This version was written in FORTRAN-90 with an improved graphical user interface written in Java. It was therefore able to run on a variety of operating systems, including Windows 10, Linux, and Mac. This feature is important moving forward because the Visual Basic implementation of the original code is obsolete and does not run on any supported operating system. Unlike the original code, the MSTAR'12 code did not include side streams, a valuable resource much desired by IAEA.

This project is designed to update the MSTAR'12 code with four principal objectives in mind: (1) to correct some errors in calculation, (2) to provide more useful output, to (3) establish formal quality control evaluations, and most importantly (4) to include side feed and side product streams. Guidance for users is found in Section 2, where both input and output options are discussed. Development and comparison of sample problem results are given in Section 3, which also provides an overview of the strengths and limitations of the new coding. The basic mathematical formulation is described in Section 4 and relies on the previously unpublished derivations of Von Halle [5], which were the foundation for the original Visual Basic code. Some of the detailed derivations and equations are relegated to appendixes. The strategy for more generalized input is described in Section 5, together with some of the additional capabilities that this affords the user.

\section{USER'S MANUAL}

Every computational algorithm requires that something be known about the processes to be modeled. Hence, sufficient input must be supplied to ensure that a solution (i.e., a mathematical description of the process) exists and is unique. (Purists will also desire stability, which is not an issue in the present model.) A description of the mathematical justification for various input requirements is given in Sections 4 and 5. In this section, we simply describe the requirements needed to successfully run the MSTAR2019 code. The original MSTAR code [6] required specific inputs as described in the second column of Table 1. In MSTAR'12 [9], these requirements were relaxed somewhat to allow more flexible inputs.

Table 1. Input requirements for previous code versions.

\begin{tabular}{|c|c|c|}
\hline Variable Description & Original MSTAR & MSTAR'12 \\
\hline Separation factor & Required & Not needed if stage number given \\
\hline Feed assays & All isotopes required & \multirow{3}{*}{$\begin{array}{l}\text { Flexible: at least one assay for each } \\
\text { isotope, minimal number required }\end{array}$} \\
\hline Tails assay & ${ }^{235} \mathrm{U}$ required & \\
\hline Product assay & ${ }^{235} \mathrm{U}$ required & \\
\hline Flow rates & Feed or product required & Feed or product required \\
\hline Side feed assays & All isotopes required & $\mathrm{N} / \mathrm{A}$ \\
\hline Side product assays & ${ }^{235} \mathrm{U}$ required & $\mathrm{N} / \mathrm{A}$ \\
\hline Assay ordering for ${ }^{235} \mathrm{U}$ & $\begin{array}{l}\text { Tails }<\text { feed }<\text { side feed }<\text { side } \\
\text { product }<\text { top product }\end{array}$ & Tails $<$ Feed $<$ Product \\
\hline
\end{tabular}




\begin{tabular}{|l|l|l|}
\hline $\begin{array}{l}\text { Stage numbers for feed(s) and } \\
\text { product(s) }\end{array}$ & $\begin{array}{l}\text { Unspecified on input, calculated for } \\
\text { output }\end{array}$ & $\begin{array}{l}\text { Could be specified instead of } \\
\text { separation factor or some assays }\end{array}$
\end{tabular}

For example, the separation factor could be omitted if numbers of stages in either enriching section, stripping section, or entire cascade were specified. In MSTAR2019, the rigid requirements of the original MSTAR are not required; however, the addition of side streams creates complications not encountered in MSTAR'12. This version still allows flexible inputs, and many are described in Sections 3.1.3, 3.1.4, and 5.1.2. However, there are many possible combinations, and they cannot all be described in detail. The code has therefore included a number of error and warning messages to guide the user to supply correct data that will result in a successful calculation. Experience has shown that the basic input requirements of the original MSTAR will always provide excellent results if the desired enrichment is physically achievable. Thus, we recommend that if possible, the user should supply such input. However, input alternatives are also available that may greatly enhance the understanding of a particular process.

\subsection{SPECIFICATIONS FROM THE IAEA}

Inasmuch as development of this tool has been sponsored by IAEA, it has been tailored according to its specific requests for input and output, default settings, and convenient access to results.

\subsubsection{Input Requirements}

Five default enrichment cases have been included that can be easily accessed through the graphical user interface (GUI). These cases are shown in Table 2 and include most of the situations commonly encountered by IAEA analysts:

1. Reprocessed-Reprocessed feed from 3.5\% low-enriched uranium, irradiated in a light water reactor to the point its enrichment in ${ }^{235} \mathrm{U}$ is similar to natural uranium (NU)

2. $\mathrm{CNU}$ - Commercial-grade natural uranium (CNU) is NU contaminated with $20 \mathrm{ppm}^{236} \mathrm{U}$

3. NUeq- $0.35 \%$ depleted uranium (DU) from tails stream of case 2 above (CNU), having $0.011 \%{ }^{236} \mathrm{U}$, and re-enriched to $0.72 \%$ with $0.15 \%$ tails.

4. irradNU_-Irradiated NU, subsequently reprocessed for re-enrichment of uranium isotopes. Burnup sufficient to produce $5 \%{ }^{240} \mathrm{Pu}$ in a Hanford-type reactor

5. Natural feed-NU with no modification.

The feed flow rate units are arbitrary, and the separation factor is a standard quantity that does not necessarily reflect any particular machine. Any of these quantities can easily be changed by the user as described in Section 2.2. No side streams are included in these standard cases, but they can easily be added.

Table 2. Default inputs.

\begin{tabular}{|c|c|c|c|c|c|c|c|}
\hline \multirow{2}{*}{ Description } & \multicolumn{3}{|c|}{ Feed Assays } & \multirow{2}{*}{$\begin{array}{l}\text { Tails } \\
{ }^{234} \mathbf{U}\end{array}$} & \multirow{2}{*}{$\begin{array}{c}\text { Product } \\
{ }^{235} \mathrm{U}\end{array}$} & \multirow{2}{*}{$\begin{array}{l}\text { Feed } \\
\text { Flow }\end{array}$} & \multirow{2}{*}{$\begin{array}{c}\text { Sep. } \\
\text { Factor }\end{array}$} \\
\hline & ${ }^{234} \mathbf{U}$ & ${ }^{235} \mathbf{U}$ & ${ }^{236} \mathbf{U}$ & & & & \\
\hline Reprocessed feed (RU) & 0.02 & 0.78 & 0.49 & 0.35 & 3.5 & 1 & 1.5 \\
\hline $\mathrm{NU} / \mathrm{CNU}$ & 0.0054 & 0.72 & 0.002 & 0.35 & 3.5 & 1 & 1.5 \\
\hline NUeq & 0.0039 & 0.72 & 0.002 & 0.35 & 3.5 & 1 & 1.5 \\
\hline irradNU & 0.0052 & 0.6445 & 0.0133 & 0.35 & 3.5 & 1 & 1.5 \\
\hline Natural feed & 0.0054 & 0.7204 & 0 & 0.35 & 3.5 & 1 & 1.5 \\
\hline
\end{tabular}


Input parameters are passed to the MSTAR executable by way of a text input file with space-separated parameters. See APPENDIX G for the file format and examples.

\subsubsection{Output File Requirements}

A standard output summary is generated for each run and formatted to IAEA specifications; an example appears in Table 3. The file is in comma-separated value (CSV) format with the first row containing only one entry describing the case that was run. All other rows have stage number in the first column followed by ${ }^{232} \mathrm{U}$ through ${ }^{238} \mathrm{U}$ assays in the following columns. If an isotope is not included in a run, the corresponding column is left blank. The first row contains waste assays, the last row contains product assays, and all other rows contain feed assays. This table represents the total range of assays expected to exist in the cascade.

Table 3. Example summary file output.

\begin{tabular}{|r|c|r|r|c|c|}
\hline \multicolumn{7}{|c|}{$\mathbf{0 . 7 2 0 4 \%}$ U235 enriched to 3.5\% 0.35\% tails } \\
\hline Stage & U232 & U234 & U235 & U236 & U238 \\
\hline 1 & - & 0.001515 & 0.32687 & - & 99.672 \\
\hline 1 & - & 0.002002 & 0.40004 & - & 99.598 \\
\hline 2 & - & 0.002644 & 0.4895 & - & 99.508 \\
\hline 3 & - & 0.003513 & 0.59885 & - & 99.398 \\
\hline 4 & - & 0.004694 & 0.73244 & - & 99.263 \\
\hline 5 & - & 0.0059 & 0.89557 & - & 99.099 \\
\hline 6 & - & 0.007444 & 1.0946 & - & 98.898 \\
\hline 7 & - & 0.009433 & 1.3373 & - & 98.653 \\
\hline 8 & - & 0.012007 & 1.6329 & - & 98.355 \\
\hline 9 & - & 0.015355 & 1.9925 & - & 97.992 \\
\hline 10 & - & 0.019732 & 2.4294 & - & 97.551 \\
\hline 11 & - & 0.025482 & 2.959 & - & 97.016 \\
\hline 11 & - & 0.032869 & 3.5998 & - & 96.367 \\
\hline
\end{tabular}

\subsubsection{Installation and Configuration Requirements}

Per IAEA requirements, the MSTAR and MSTAR-GUI executables are built for Windows 64-bit. They are installed into $\mathrm{C}: \backslash$ Program $\mathrm{Files}$ by default and a silent install option is available. Application information is stored under the user's home folder (e.g., C: \Users \USERNAME \.mstar) in the user's section of the registry. No executable or dynamic link library (DLL) is installed or run from the user's home folder and no scripts are dynamically generated. The MSTAR executable can be run directly without going through the user interface or another application can generate input files and pass them to MSTAR. The necessary input files are described in APPENDIX G. Linux and MacOS versions of both MSTAR and MSTAR-GUI are also available. 


\subsection{GRAPHICAL USER INTERFACE}

The MSTAR GUI is divided into two sections, which are selected from the two buttons along the left side of the window (Figure 2). The Edit cascade section is used for configuring inputs for submitting to MSTAR. The Run section is used for running the MSTAR executable and viewing outputs in various tables and charts and saving those to disk. The button for the currently selected section is greyed out.

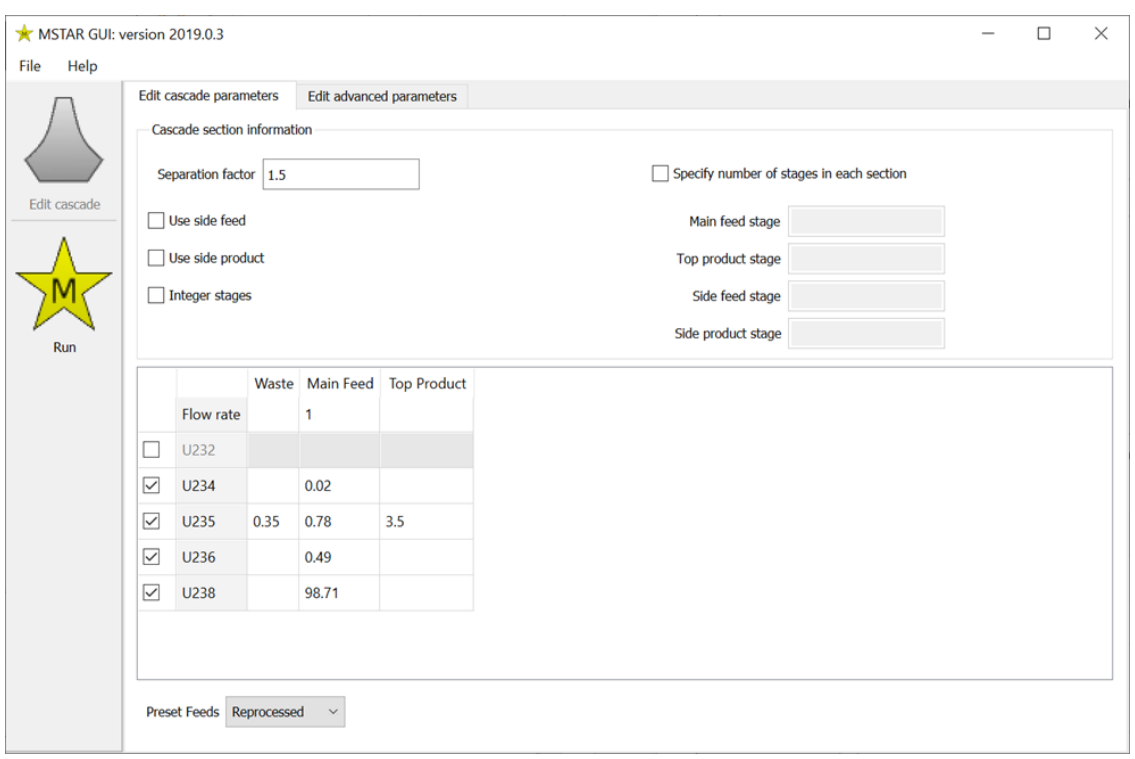

Figure 2. Edit cascade $\rightarrow$ Edit cascade parameters.

\subsubsection{Edit cascade}

The Edit cascade section contains two tabs. Most important properties are on the Edit cascade parameters tab (Figure 2), including the separation factor, feed/product stages, and input assays. Stages numbers are not defined by default but are calculated; hence, the stage fields are disabled. For all input fields, a blank entry indicates the value is not set when running MSTAR. 
A number of check boxes on this tab will enable modifications and additional options. Checking Use side feed or Use side product enables their respective stage fields (Figure 3). Checking Specify number of stages enables the stage fields so these values can be entered. Checking Integer stages restricts stage inputs to integer numbers and will produce output with integer numbers of stages in each region of the cascade. Input assays are provided in the table on the bottom half of the window. If Use side feed or Use side product is checked, a corresponding column is added to the table for inputting these assays. Checking or unchecking an isotope in the leftmost column of the table will add or remove that isotope from the input set. However, if the isotope is the matched or key component (see Sections 2.3.1 and 4.2 for explanations of these terms), it cannot be unchecked until changing the relevant field in the Edit advanced parameters tab (Figure 4). Below the input assays table is the Preset feeds drop-down selection. Changing this selection updates the input assays table with stored values from one of the sets in Table 2. If the input assays table is modified by the user, this drop-down changes selection to User specified.

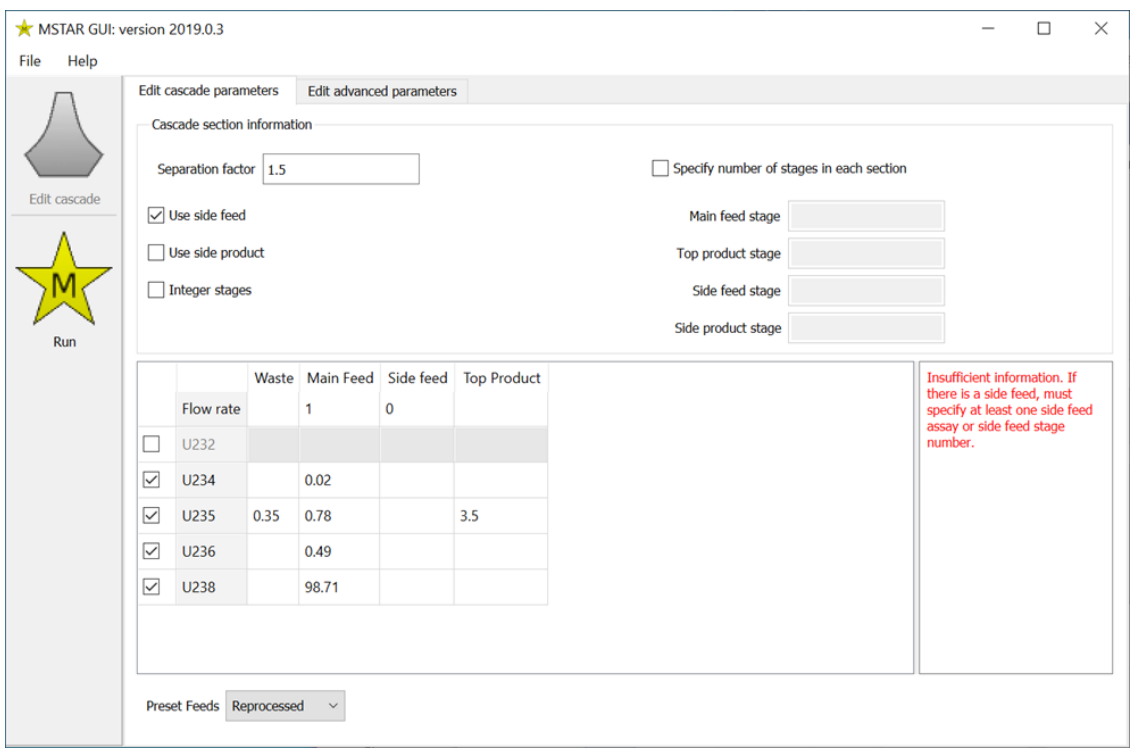

Figure 3. Edit cascade $\rightarrow$ Edit cascade parameters. Side feed checked. 
The Edit advanced parameters tab has fields for machine configuration and matched/key components (Figure 4). It is recommended that the novice user leave the matched/key components alone; an example of changing these is given in Appendix F. If individual machine flow rates are known, or total numbers of machines are known, these can be entered here. These entries are explained further in Section 2.3.

If incomplete or invalid values are entered for any of the inputs, there may be an error message in red on the right side of the Edit cascade parameters tab (Figure 3). Until the issues are fixed, MSTAR will not be run. The MSTAR GUI cannot catch all possible invalid input combinations, though it tries to catch many that are known. It is still possible to create certain input sets that meet minimal input requirements, but cause an execution-time error when MSTAR is run. Such messages are explained in the next section.

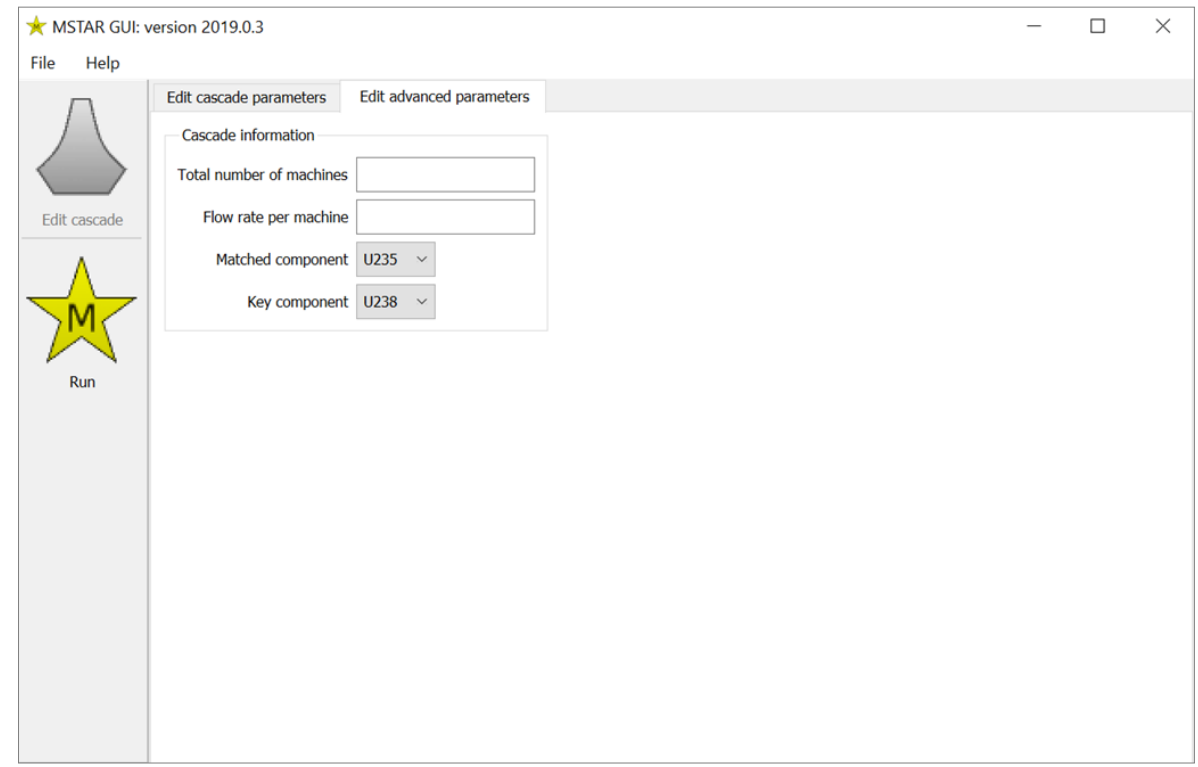

Figure 4. Edit cascade $\rightarrow$ Edit advanced parameters. 


\subsubsection{Running MSTAR}

Clicking the Run button from the left column immediately runs the MSTAR executable if all inputs are valid. If inputs are not valid, an error dialog will pop up with the same error as shown in red text on the Edit cascade section. If the executable is run, a brief summary of outputs can be found in the MSTAR Output tab (Figure 5). If there are errors running MSTAR, they are shown in the MSTAR Error tab.

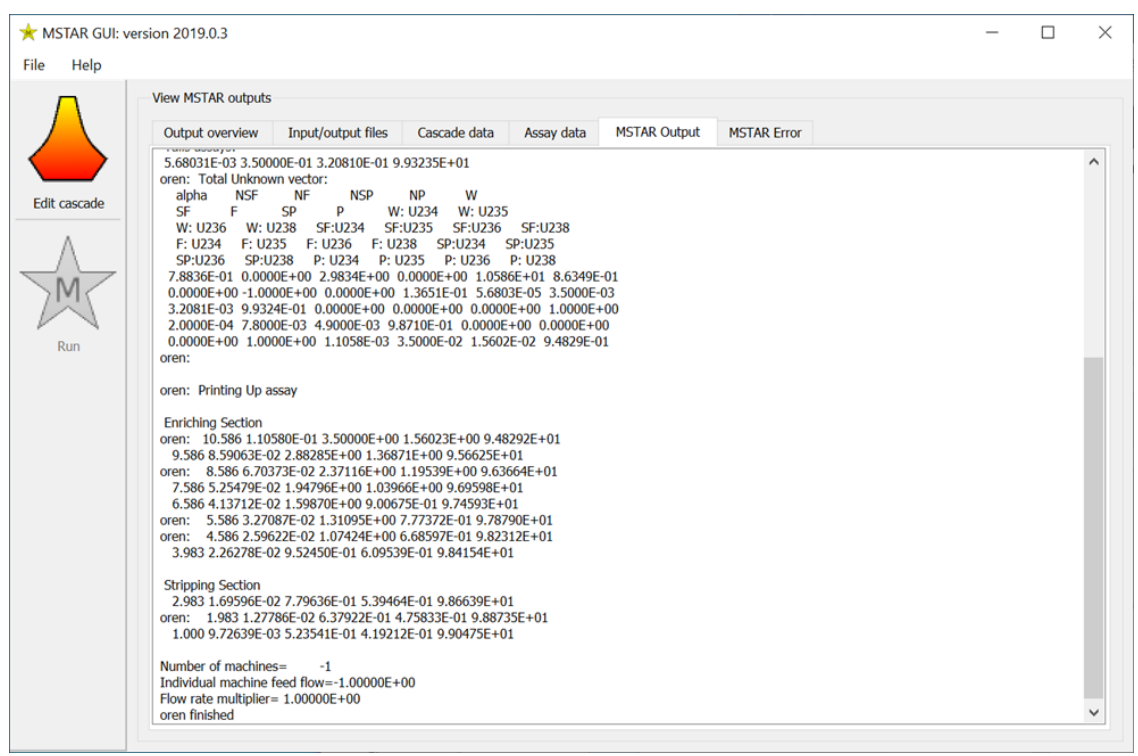

Figure 5. Run MSTAR.

\subsubsection{View output}

After running MSTAR, the View output section will show the results of the run organized into charts and tables across several tabs. The Output overview tab is shown initially (Figure 6), which contains some basic cascade and machine information along with output assays. There are two buttons at the bottom for saving the output summary file or opening it directly in MS-Excel.

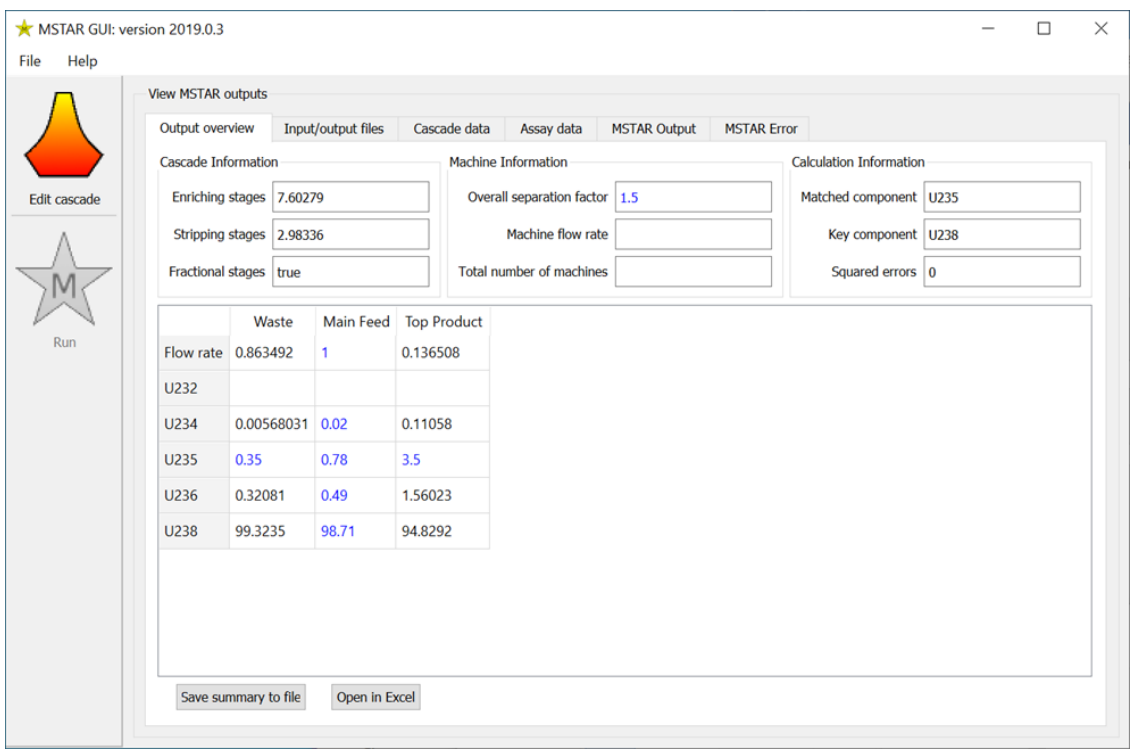

Figure 6. View output $\rightarrow$ Output overview. 
The Input/output files tab has subtabs for all output files generated by MSTAR (Figure 7). There is a Save to file button at the bottom to allow saving a CSV file to disk. The Summary file and Long summary file tabs also have an Open in Excel button (Figure 8), which will directly open as a spreadsheet. The Error file tab is disabled unless there was an error running MSTAR.

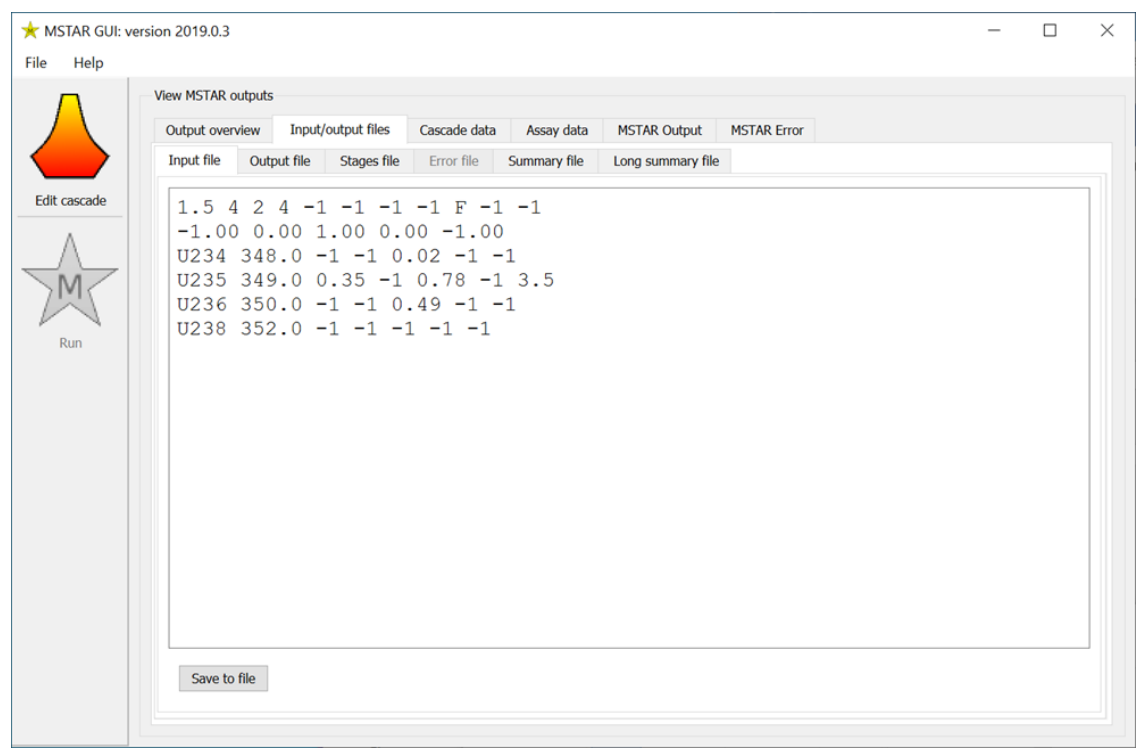

Figure 7. View output $\rightarrow$ Input/output files $\rightarrow$ Input file.

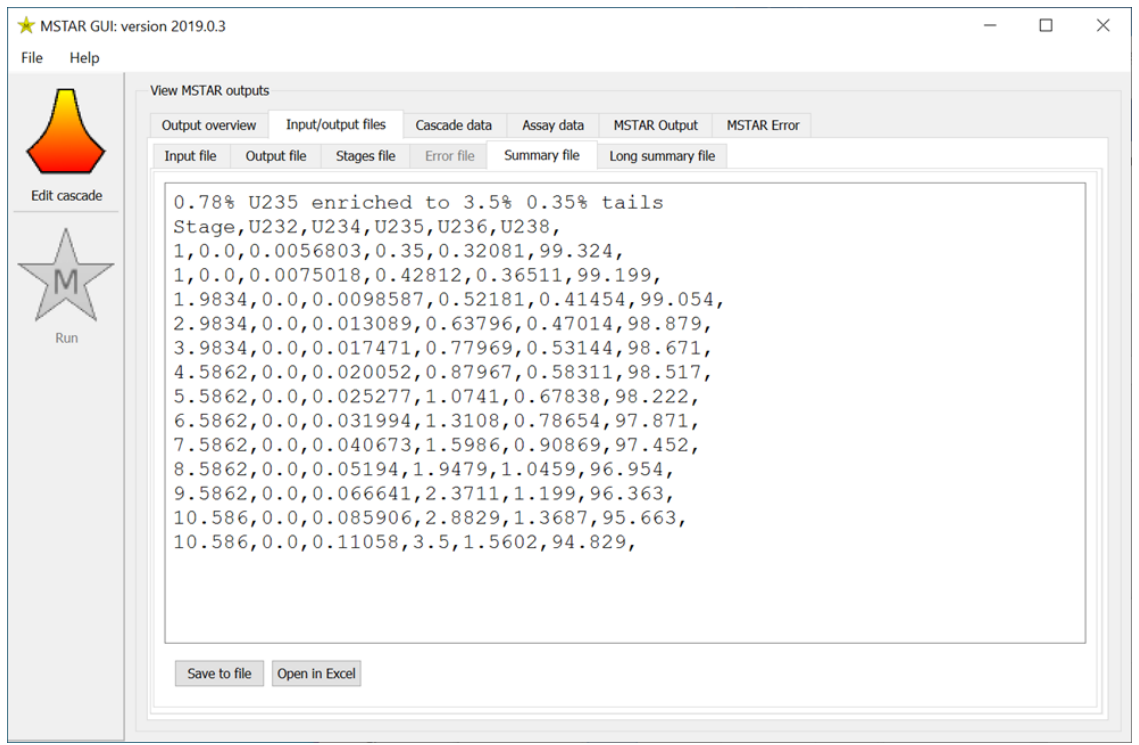

Figure 8. View output $\rightarrow$ Input/output files $\rightarrow$ Summary file. 
The Cascade data tab has four subtabs. The first is Cascade table, which shows stages, flow rates, machines, and cuts (Figure 9).

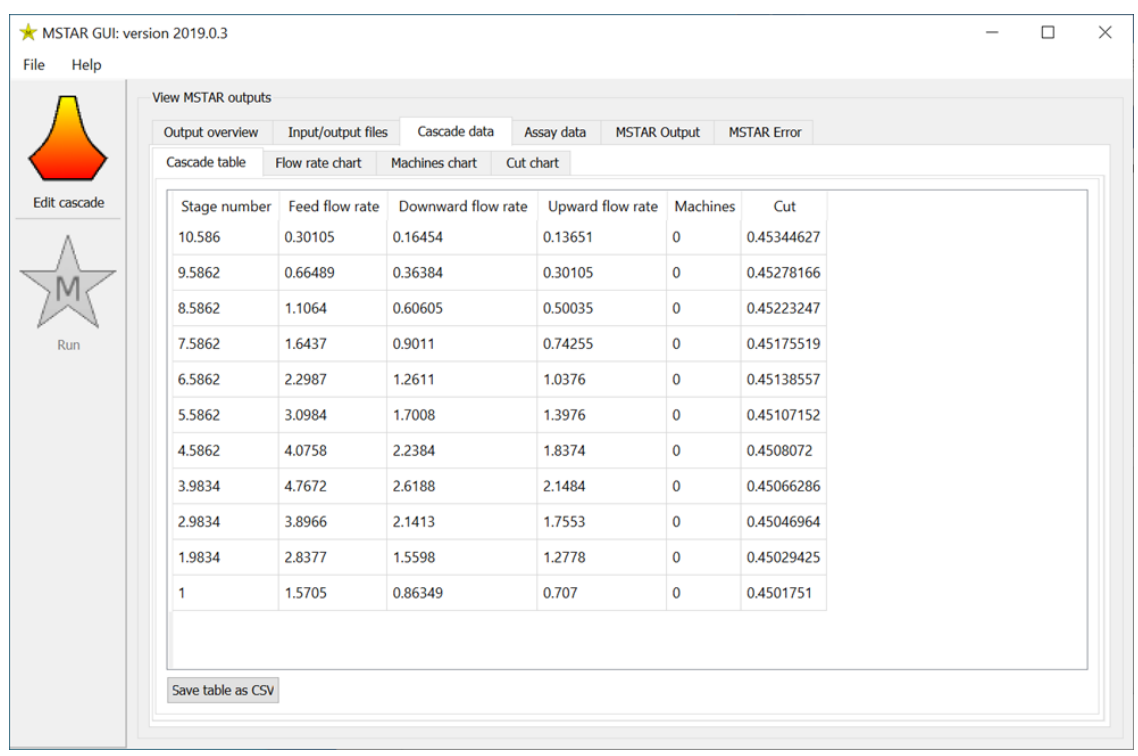

Figure 9. View output $\rightarrow$ Cascade data $\rightarrow$ Cascade table.

The other three are Flow rate chart, Machines chart, and Cut chart, and each plots the corresponding data from the cascade table (Figure 10-Figure 13). The table can be saved to a CSV file, and the charts can be saved as PNG files with save buttons below the table and charts, respectively. The charts are interactive with mouse click-and-drag and zoom with the mouse wheel. The Reset chart button will restore a chart back to its default position and scale. The Machines chart will be a flat line unless machine properties were set in the cascade input (Figure 11 and Figure 12).

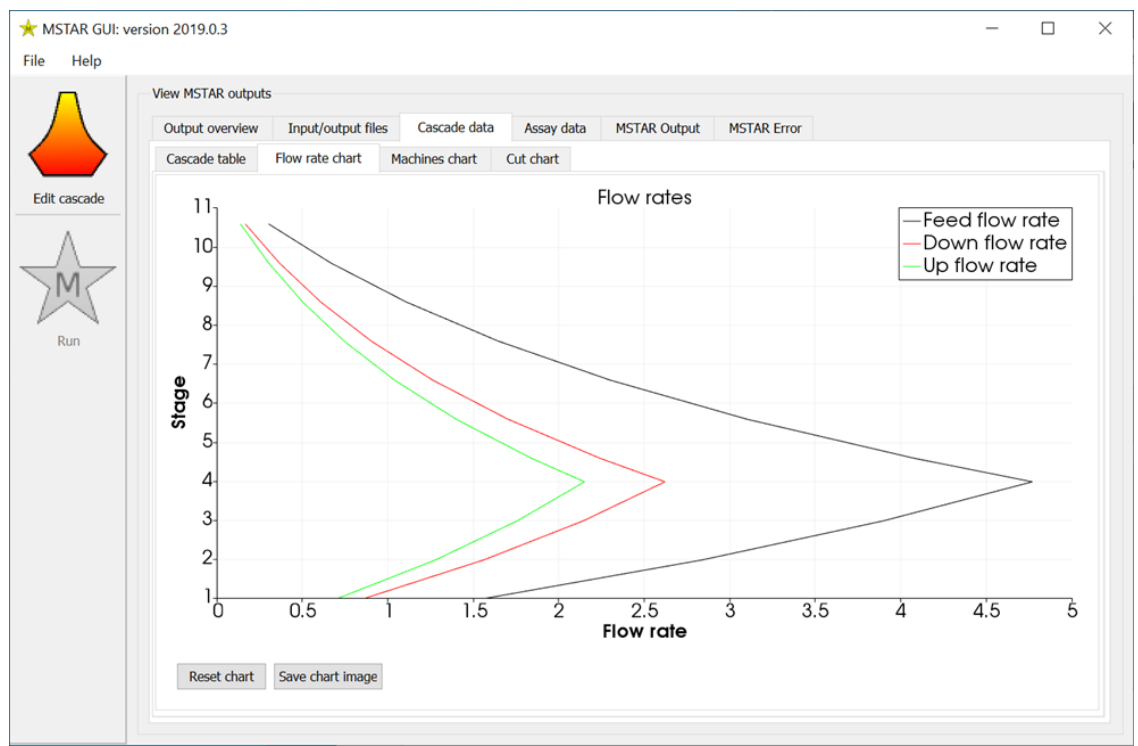

Figure 10. View output $\rightarrow$ Cascade data $\rightarrow$ Flow rate chart. 


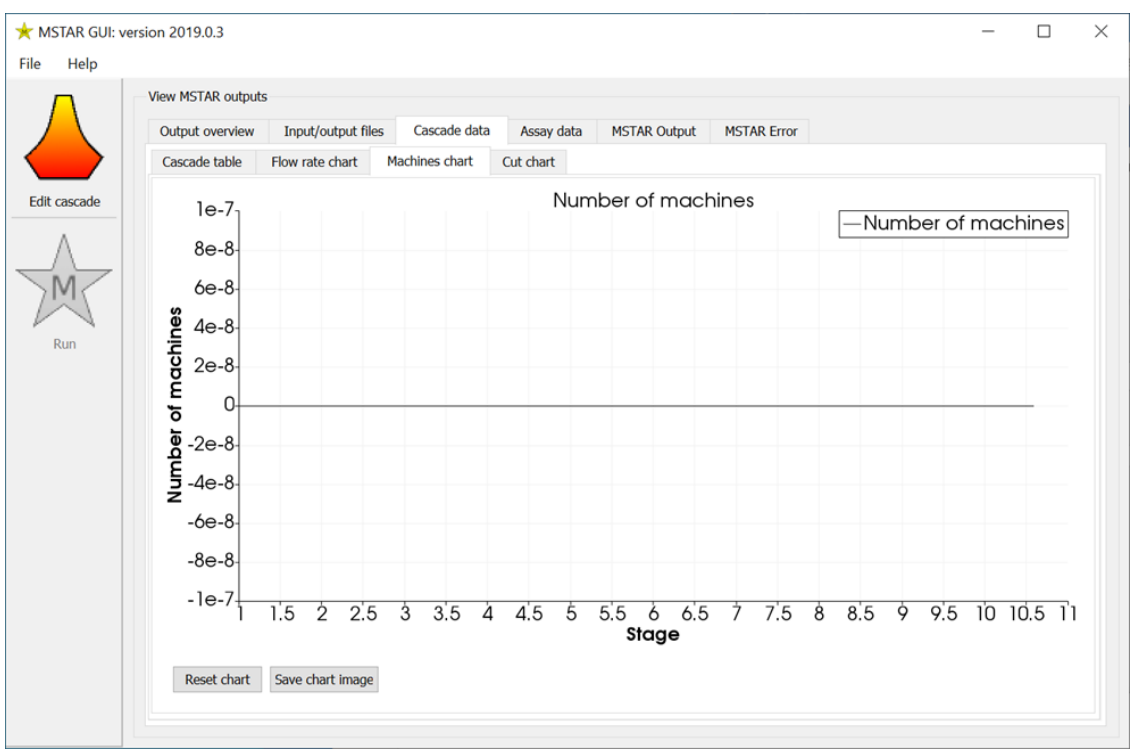

Figure 11. View output $\rightarrow$ Cascade data $\rightarrow$ Machines chart. Machines not set. Total number of machines and flow rate per machine not set.

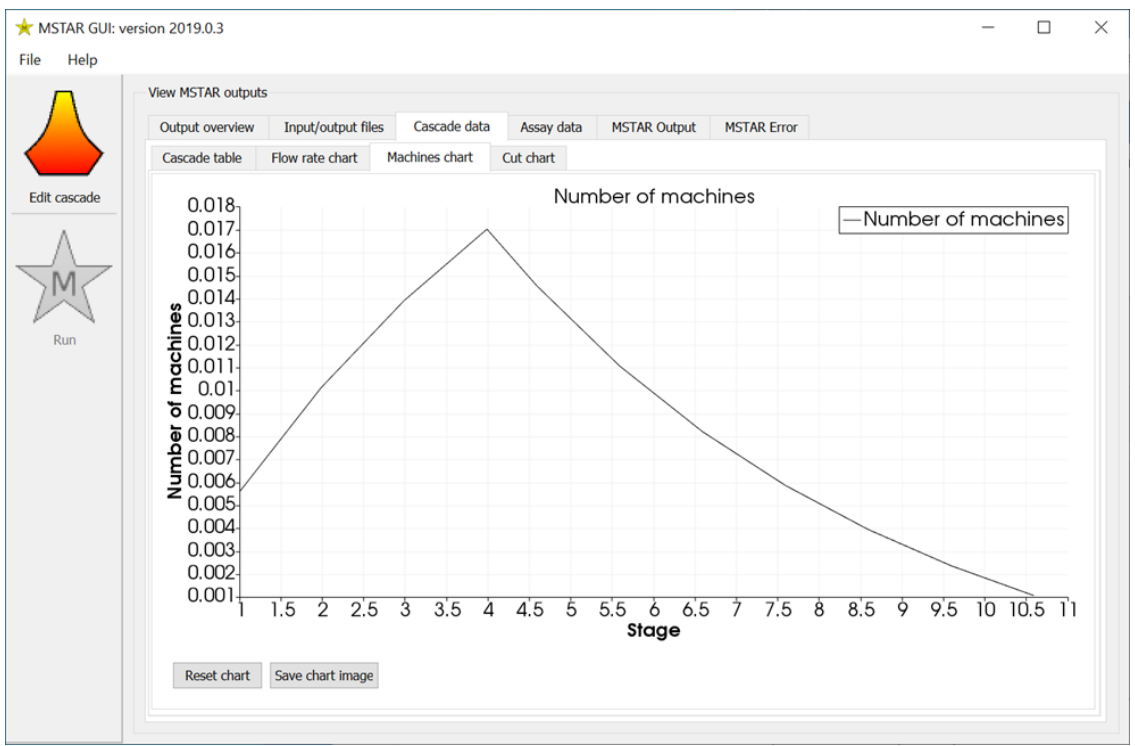

Figure 12. View output $\rightarrow$ Cascade data $\rightarrow$ Machines chart. Machines set. Total number of machines $=1,000$. Flow rate per machine $=280(\mathrm{mg} / \mathrm{s})$. 


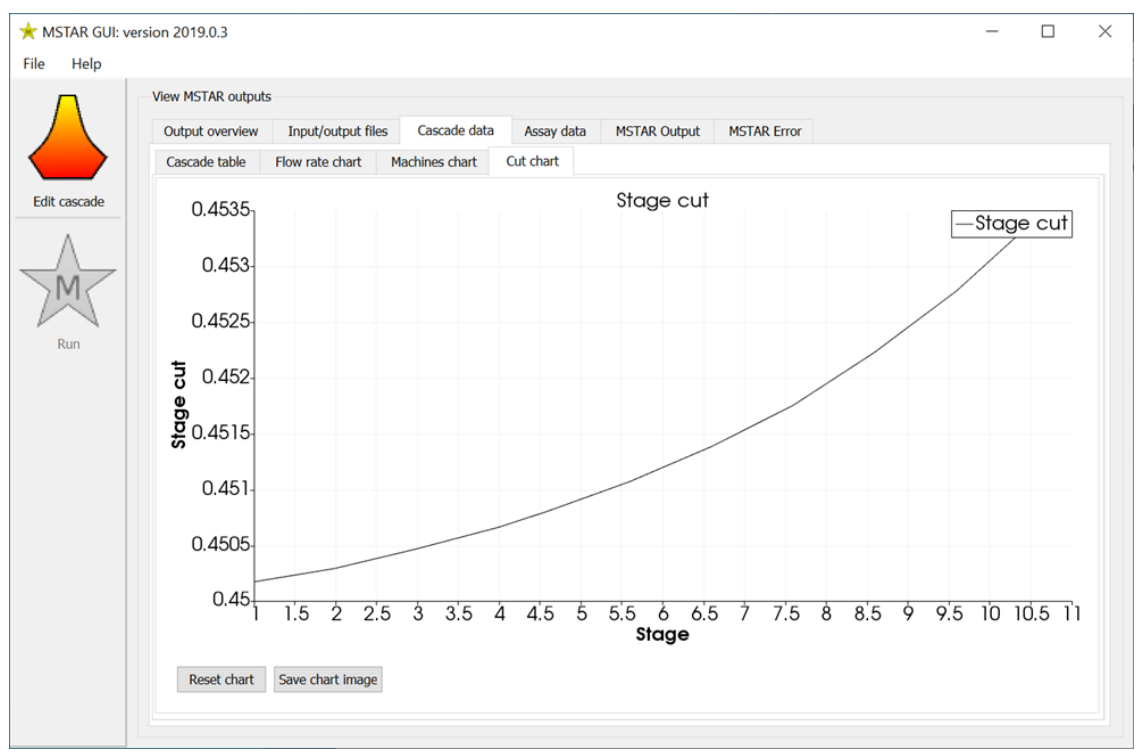

Figure 13. View output $\rightarrow$ Cascade data $\rightarrow$ Cut chart.

The Assay data tab has three subtabs. The Assay table subtab shows stages, feed, and up (product) and down (waste) assays (Figure 14).

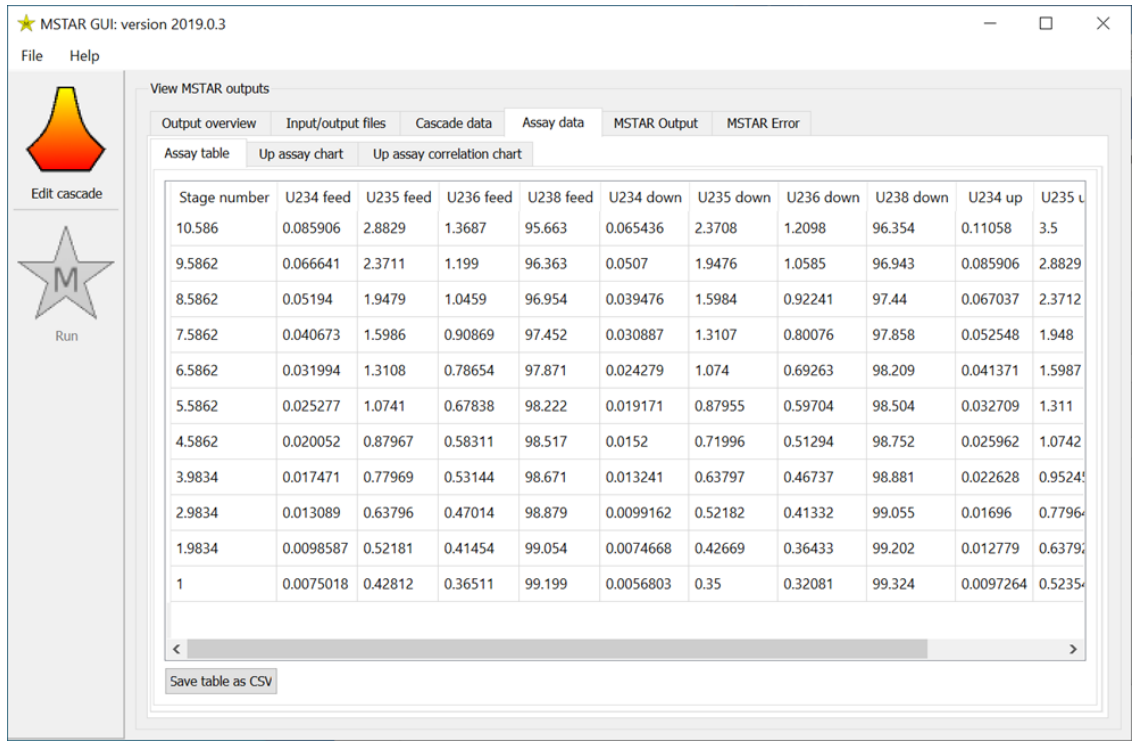

Figure 14. View output $\rightarrow$ Assay data $\rightarrow$ Assay table. 
The Up assay chart plots the atom percent for each isotope for each stage (Figure 15), The Up assay correlation chart plots the atom percent of each isotope relative to another selected as the independent axis (Figure 16). Charts can be saved with the Save chart image button at the bottom of each tab. The charts are interactive with mouse click-and-drag and zoom with the mouse wheel. The Reset chart button will restore a chart to its default position and scale.

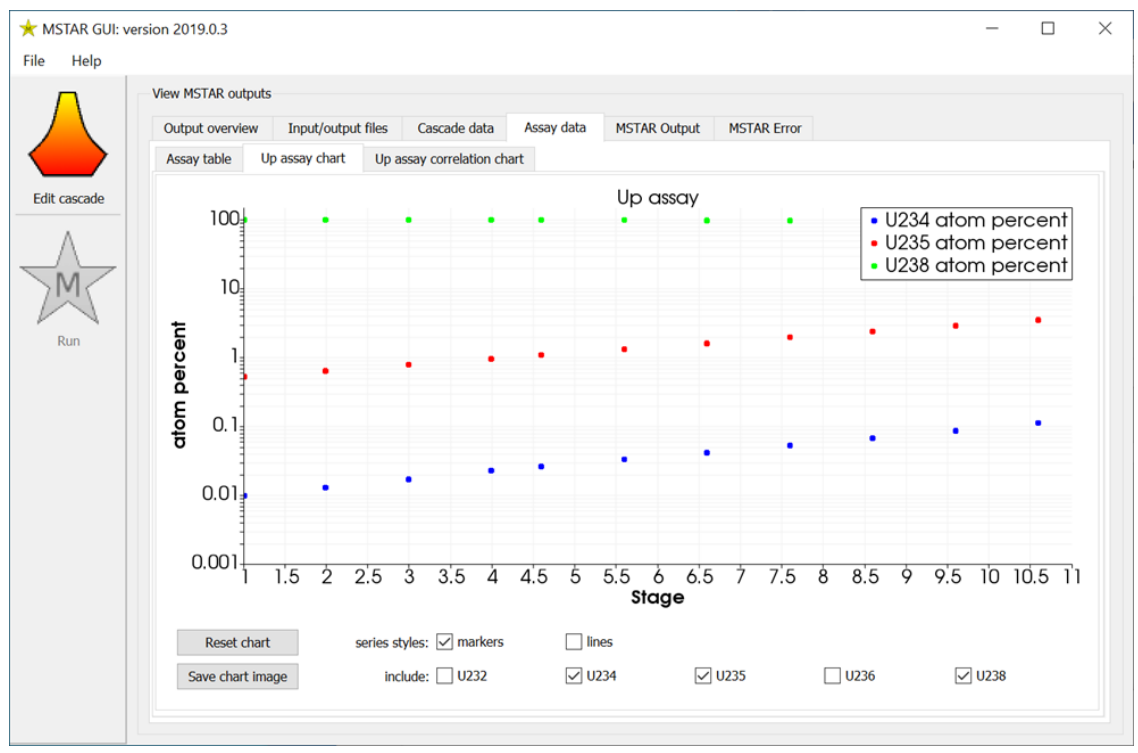

Figure 15. View output $\rightarrow$ Assay data $\rightarrow$ Up assay chart.

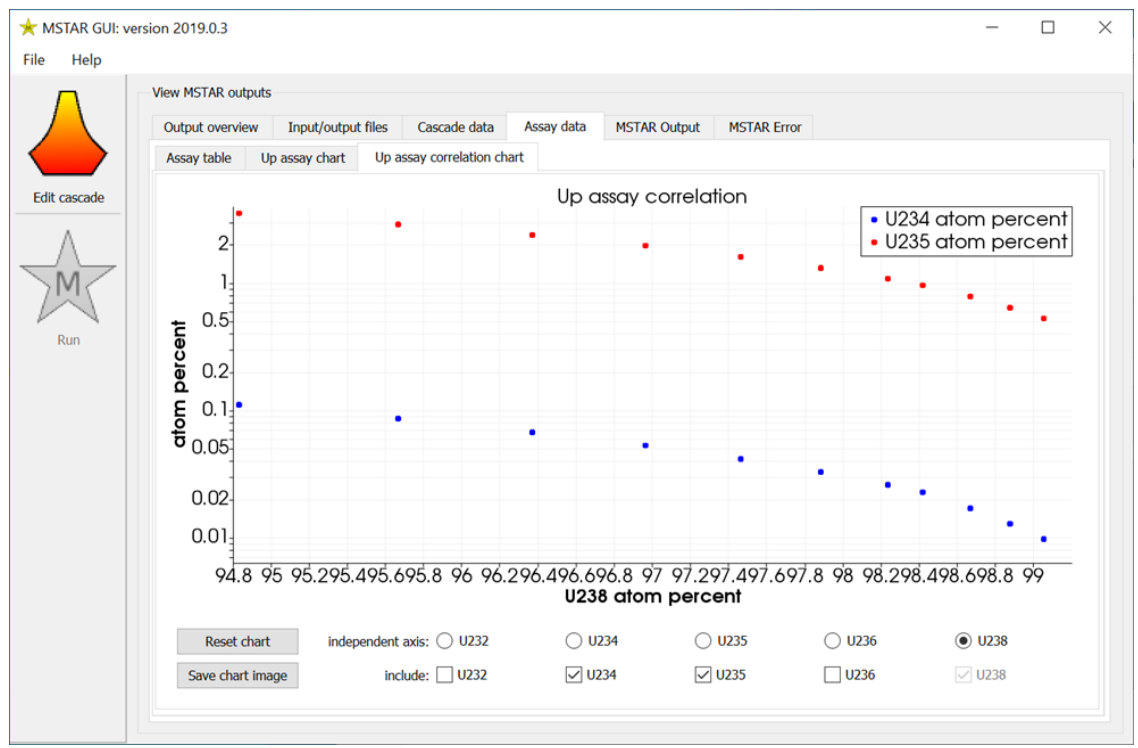

Figure 16. View output $\rightarrow$ Assay data $\rightarrow$ Up assay correlation chart. 


\subsection{ADVANCED PARAMETERS}

The MSTAR2019 code can be run successfully by simply using program defaults and perhaps changing assays or adding side streams as described in Sections 2.1 and 2.2. For most applications, this is sufficient. However, several features are included that might provide utility in unusual problems, and these are described in this section. The user is encouraged to be generally familiar with the mathematical description in Section 4 before attempting the modifications described here. The advanced parameters tab on the edit screen is shown in Figure 4 and allows additional modifications to problem parameters.

\subsubsection{Key and Matched Isotopes}

The key isotope is the one against which all others are compared and should almost always be ${ }^{238} \mathrm{U}$. However, there may be occasions when a different isotope could be considered. For example, if enrichment of reprocessed uranium to very high levels is desired, it may be advantageous to make ${ }^{236} \mathrm{U}$ the key component, as described in Section 3.3.1. However, such cases are rare, and in general, the key isotope should remain as the default ${ }^{238} \mathrm{U}$.

The matched isotope is the one whose assays are matched at piping junctions (see Section 4.2) to avoid mixing losses. It is almost always assigned to ${ }^{235} \mathrm{U}$, although this need not be the case. However, if ${ }^{235} \mathrm{U}$ is present, and is the primary focus of the enrichment operation, it is prudent to leave this setting as the default.

Note, the separation factor always describes separation between the matched and key components. Hence, familiar separation factors usually always refer to separation of ${ }^{235} \mathrm{U}$ from ${ }^{238} \mathrm{U}$ as the matched and key components, respectively. If the key or matched components are changed, the separation factor must be changed as well. This point is discussed more thoroughly in APPENDIX F.

\subsubsection{Machine Information}

Machine information may be available on the actual machines operating in a facility. Alternatively, simulating operation of generic machines whose features are known and published openly might be useful. MSTAR2019 allows this through entries of total numbers of machines and machine flow rate. Note that the separation factor also pertains to specific machines, but whether or not other machine information is known must be specified. To ensure consistency with overall cascade flow rates, the following hierarchy is honored in determining output:

1) If neither number of machines nor machine flow rate is specified on input, then no machine output is included (Figure 11).

2) If either number of machines or machine flow rate is specified, then the other is calculated to be consistent with the specified or default external cascade flows.

3) If both the number of machines and the machine flow rate are given, then the external cascade flows are scaled from their input values to be consistent with the stagewise and total cascade flows calculated from the input machine information.

For more detail in this calculation, see Section 5.4. 


\section{SAMPLE PROBLEMS}

In this section, several sample problems illustrate the capabilities and flexibility of the code. These include standard problems typical of declared facilities, as well as undeclared operations and unusual calculations. Some of the bounds and limitations on possible calculations are also presented.

\subsection{STANDARD PRODUCTION OF LOW ENRICHED URANIUM}

This scenario consists of natural uranium enriched to $3.5 \%$ with a waste stream of $0.35 \%$ in ${ }^{235} \mathrm{U}$, a typical scenario for declared enrichment operations. This is one of the cases that can be invoked from the default menu.

\subsubsection{Base Case}

If this case is run without making any changes, results shown in Figure 17 are obtained. The entire cascade includes 10.52 total stages of which 7.94 are in the enriching section and the remainder in the stripping section.

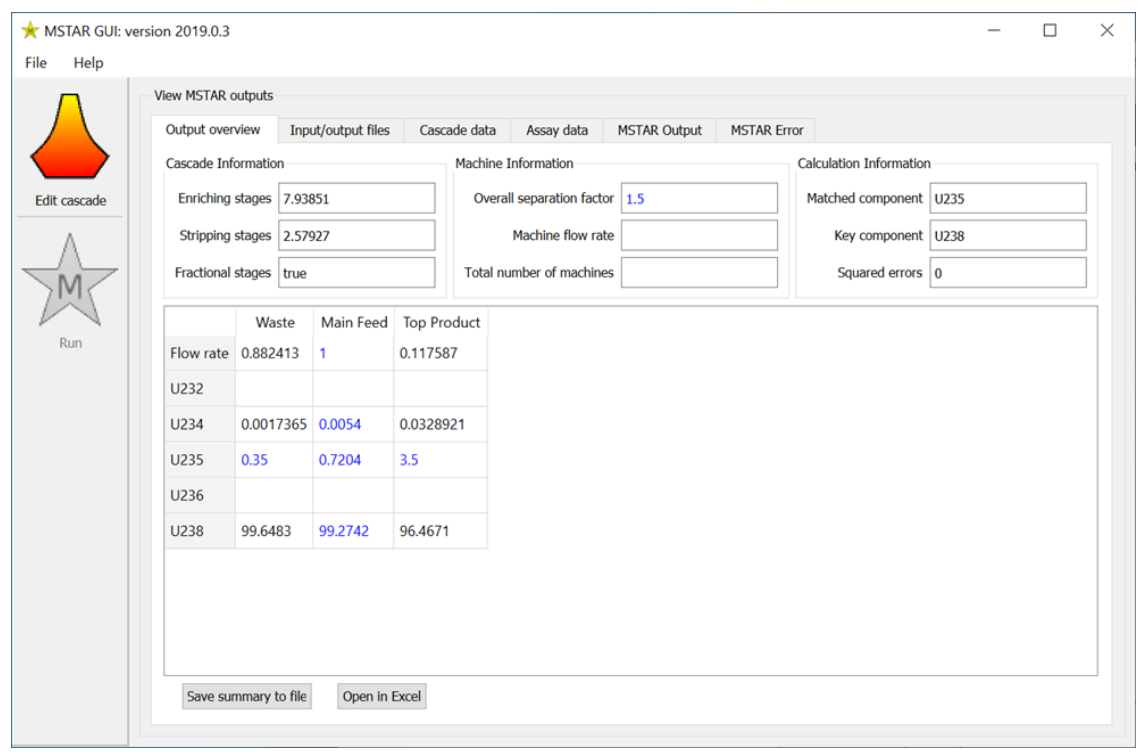

Figure 17. Base case output overview. 
Stagewise flow rates are shown in Figure 18, which are accessible from the Cascade data tab and the Flow rates chart. The machines chart is zero because no machine information has been supplied. Note that if nothing is specified, the feed flow rate default is 1 (arbitrary units), and the tails and product streams are calculated. Figure 19 shows feed assays for the entire cascade.

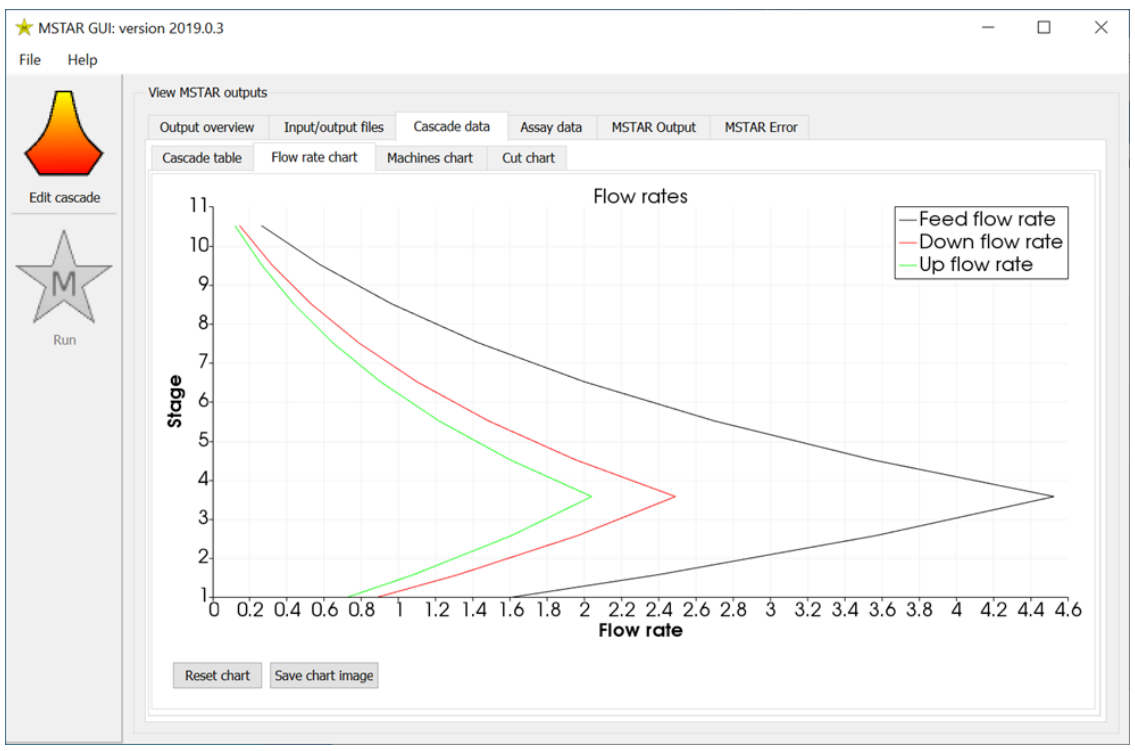

Figure 18. Base case flow rates chart.

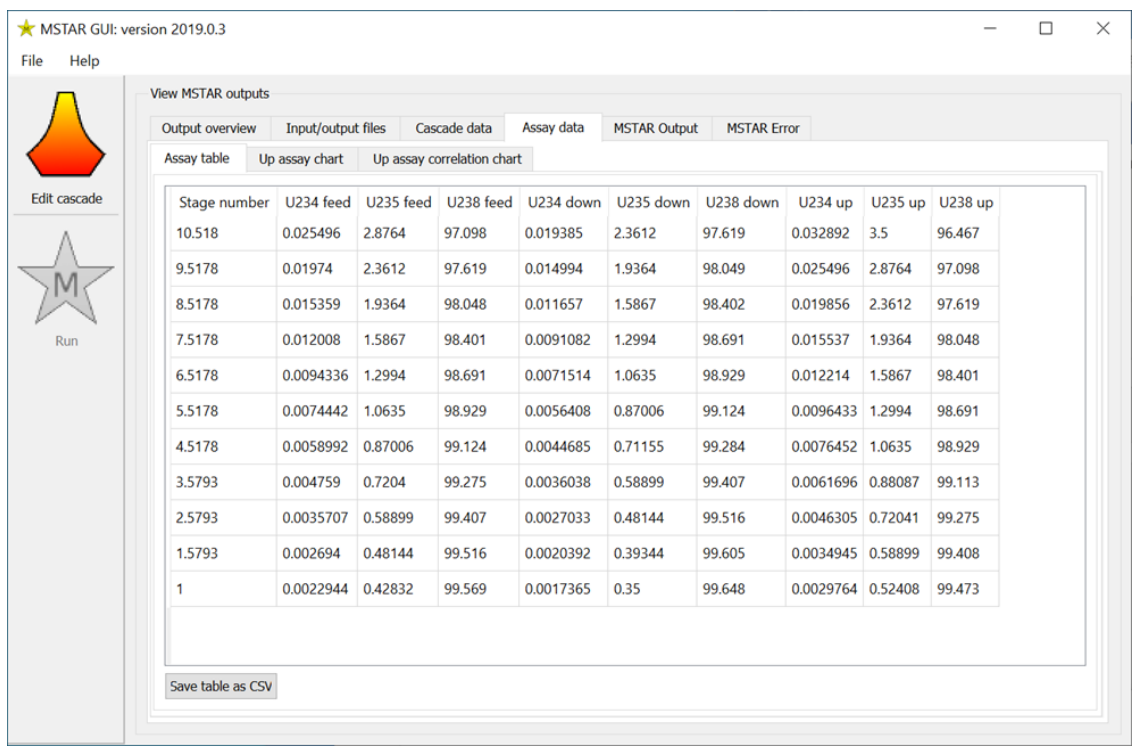

Figure 19. Base case assay data. 


\subsubsection{Inclusion of Side Streams}

As described in Section 2.2, we can add side streams to the base case scenario. If we add a side feed with flow rate 0.1 and assays of $1 \%{ }^{235} \mathrm{U}$ and $0.001 \%{ }^{234} \mathrm{U}$, the resulting cascade configuration is given in Figure 20.

Adding both a side feed and side product (each with flow rate of 0.1 ) is straightforward and gives results shown in Figure 21. As seen in the figure, the side product was specified to have a ${ }^{235} \mathrm{U}$ assay of $2 \%$ enrichment; the code calculates side product assays for the other isotopes and the stage at which the side product would occur. Note that only small changes to overall cascade behavior result. As presently configured, the feed streams are ordered so that the main feed is the one with higher assay. Consequently, in this case, the output automatically swaps the main and side feed streams.

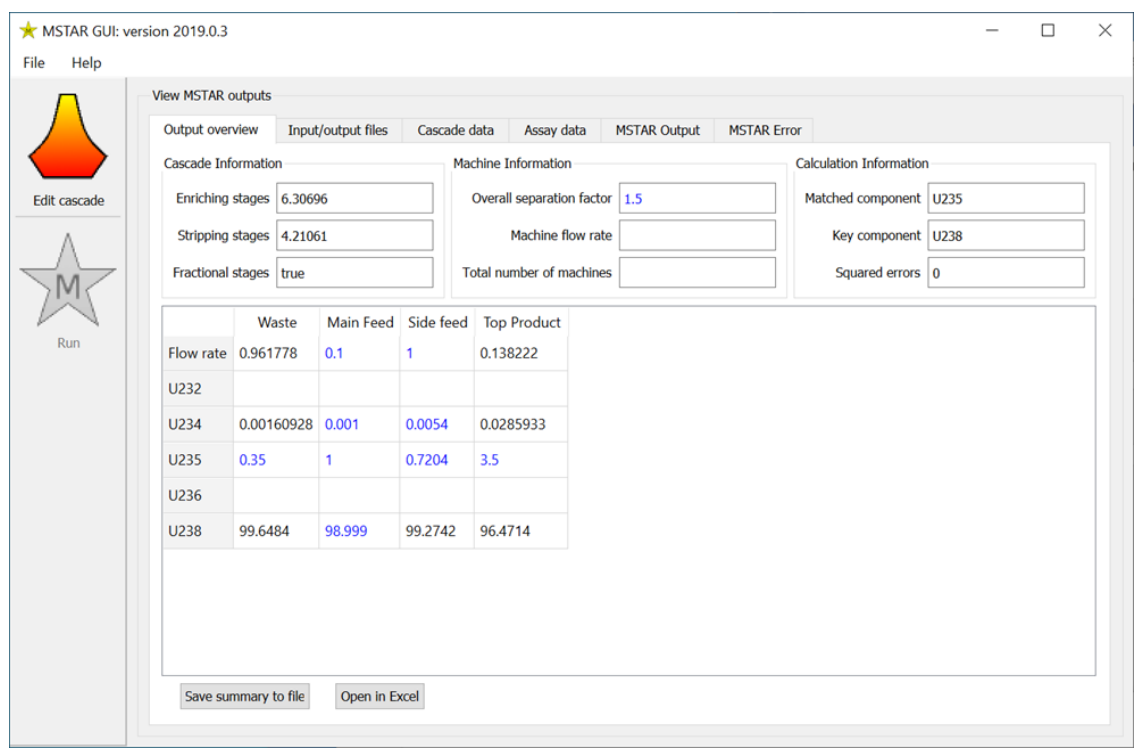

Figure 20. Overall cascade results for side feed.

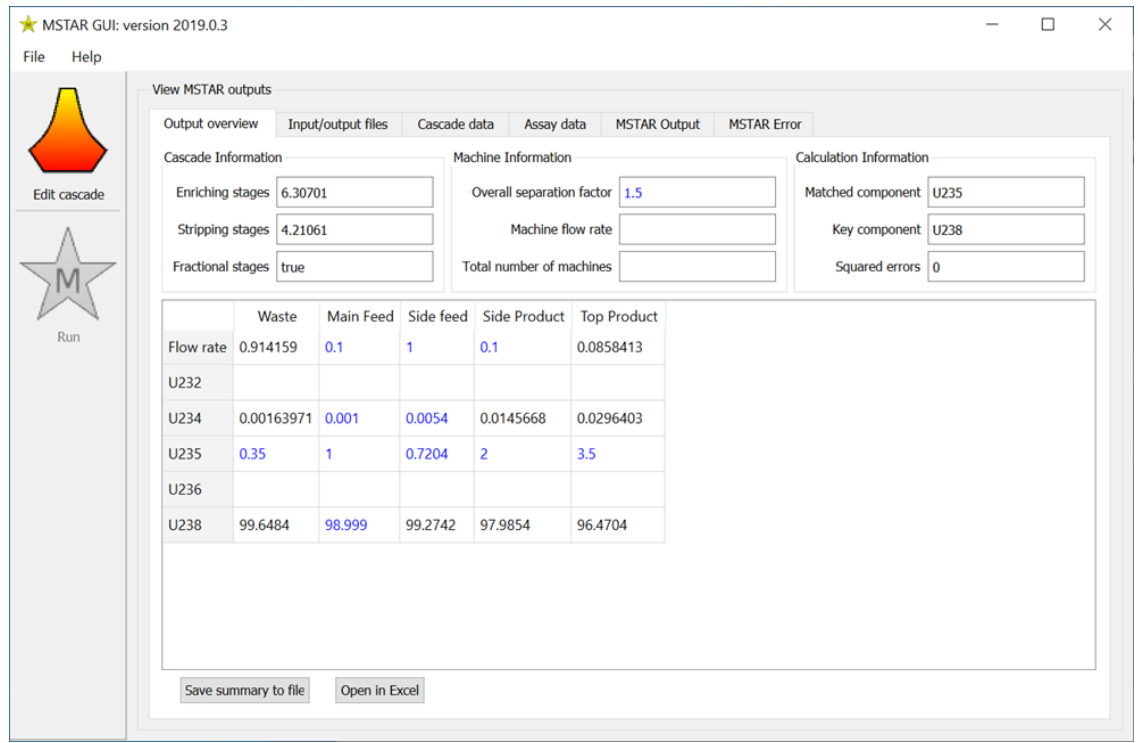

Figure 21. Overall cascade results for both side streams. 


\subsubsection{Integer Stages}

Results shown thus far have yielded noninteger numbers of stages, which is not consistent with real enrichment facilities. The MARC model as derived in Section 4 is constructed so that the calculated numbers of stages in each section are real numbers instead of integers. This approach is adequate to understand the overall cascade, as well as general stagewise features that are provided in Figure 18 and Figure 19. However, if the results need to resemble a real facility, then it is possible to impose integer stage numbers for locations of all external streams. The modifications to the algorithm are described in Section 5.2.2, and here we simply illustrate the process.

Specifying integer stages for a problem involving both side streams yields results shown in Figure 22 . Note that the final assays for ${ }^{235} \mathrm{U}$ in tails, side feed, side product, and top product vary slightly from the values originally specified. If we require exact assays and integer stages, then no solution exists for the MARC equations. By requiring integer stages, we must relax other requirements, and for MSTAR2019 this is done by allowing ${ }^{235} \mathrm{U}$ assays to vary slightly in order that the equations can be satisfied exactly. See Section 5.2.2 for further discussion.

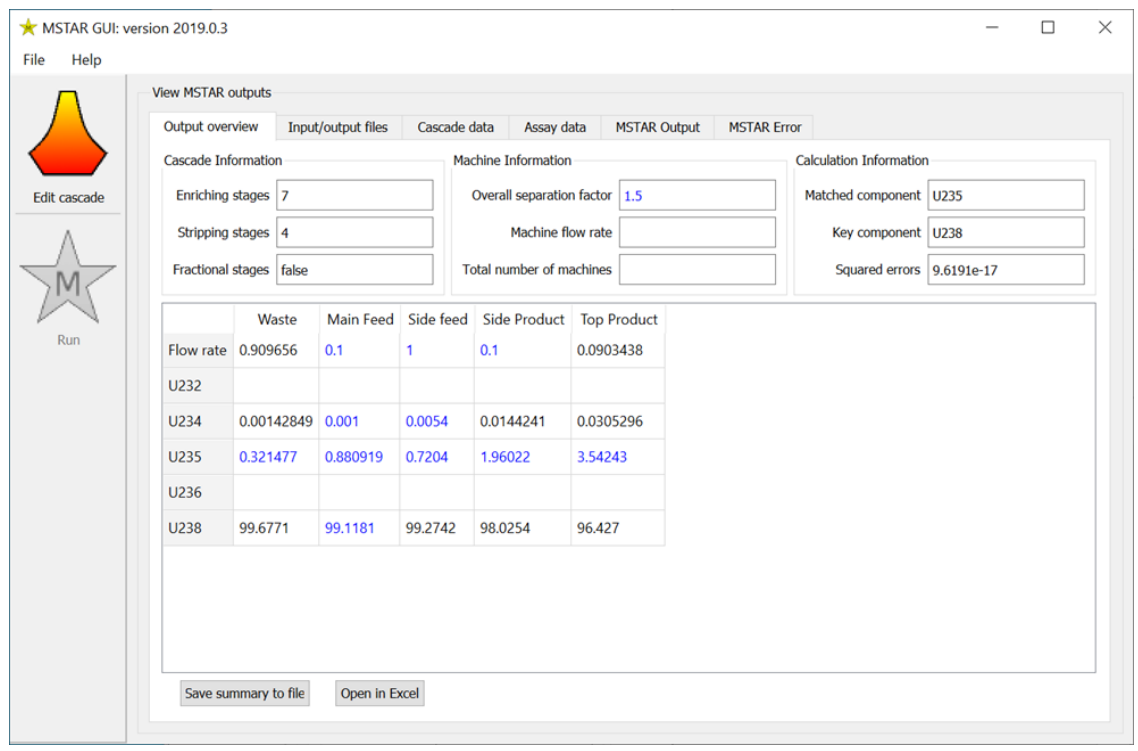

Figure 22. Output overview for side streams and integer stages. 


\subsubsection{Non-standard Input}

We have mentioned that MSTAR2019 includes input flexibility, and in this section we mention several examples of this feature. The standard input assays are ${ }^{235} \mathrm{U}$ in tails, product, and side product, together with all assays in main feed and side feed. It is possible to use different combinations of assays if necessary, although the combinations are subject to the following constraints:

1) Every isotope must have at least one assay specified in some stream.

2) Every external stream must have an assay or stage number specified.

For example, we can substitute the minor isotope ${ }^{234} \mathrm{U}$ for ${ }^{235} \mathrm{U}$ in tails and product assays, as shown in Figure 23, and the code will still provide excellent results. Various other combinations are also possible and will almost always provide good results.

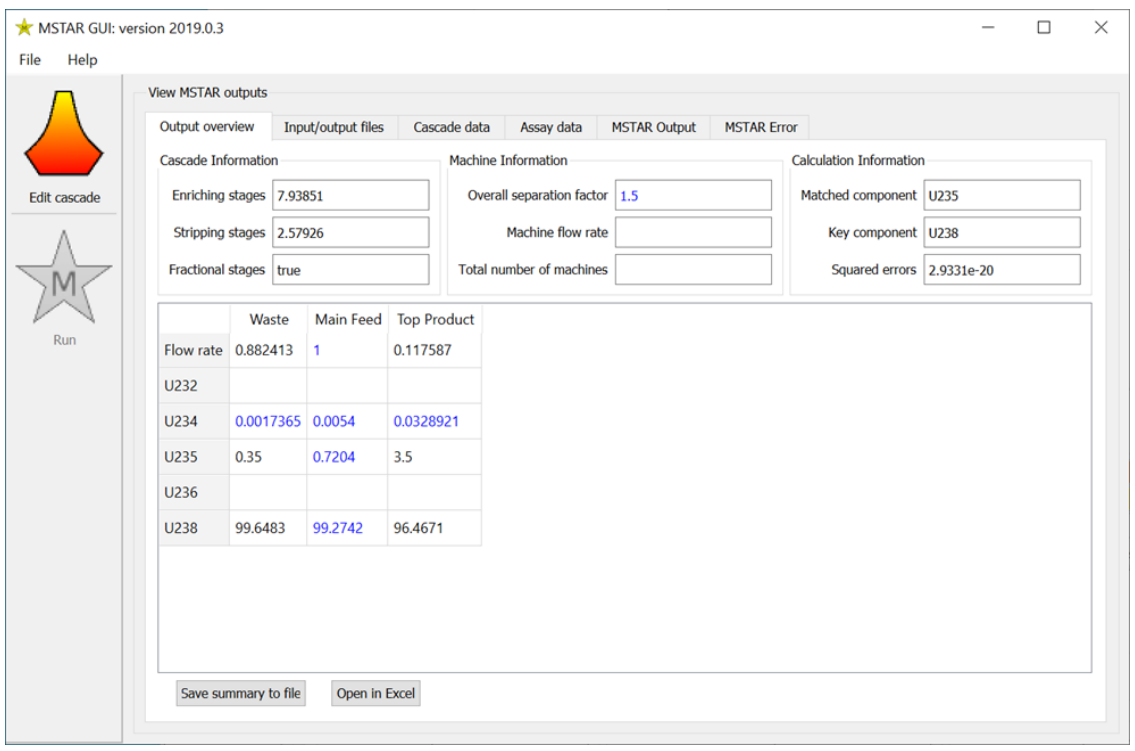

Figure 23. Output overview with minor isotope ${ }^{234} \mathrm{U}$ specified. 
The MSTAR model uses assays in the external streams to calculate the stage numbers where these streams should occur. However, it is also possible to perform an inverse calculation - specify the stage numbers, and the code will calculate the assays for streams at those stages. This is illustrated in Figure 24 and Figure 25, where we have taken the output stage numbers from Figure 21 and supplied them as input instead of ${ }^{235} \mathrm{U}$ assays in tails, side feed, side product, and top product streams. The resulting calculations reproduce the ${ }^{235} \mathrm{U}$ assays to the same precision as the input values. To account for switched main and side feeds, the main feed ${ }^{235} \mathrm{U}$ assay is set to $1 \%$ per the side feed instead of $0.7204 \%$ (see Section 3.1 .2 and Figure 21).

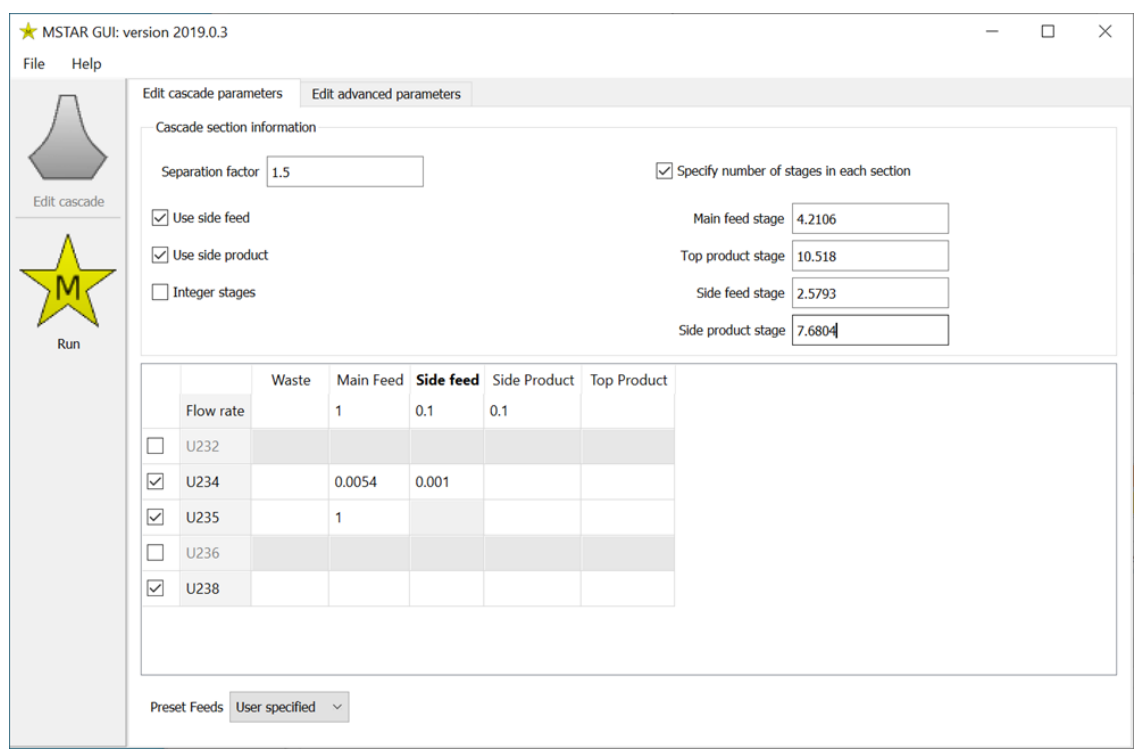

Figure 24. Input stage numbers.

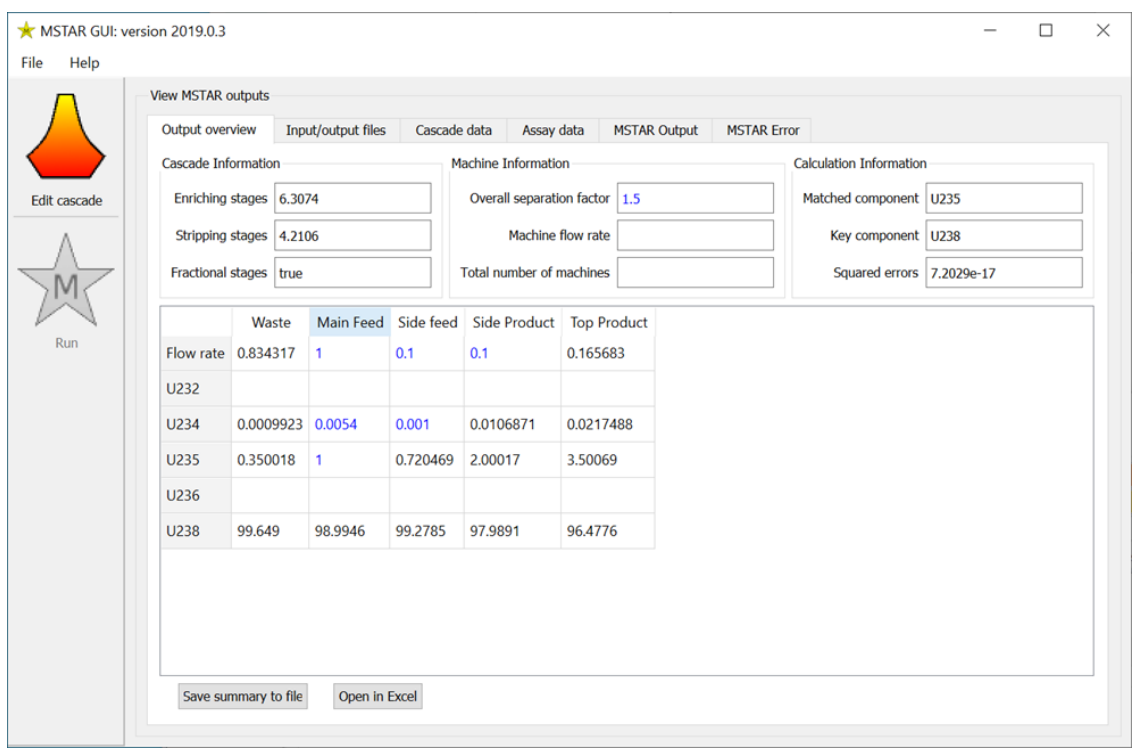

Figure 25. Output overview with stage numbers specified. 


\subsection{PRODUCTION OF HIGHLY ENRICHED URANIUM FROM RECYCLED URANIUM}

When MSTAR2019 is first launched, a default case for enrichment of recycled uranium is loaded. This is one of the standard default cases mentioned in Table 2 and is briefly described in the GUI tutorial (Section 2.2). In this section, we investigate enrichment far beyond declarations to produce a product of highly enriched uranium (HEU). This is done through two scenarios, one a single cascade configured to produce HEU, and the other by using successively higher enrichments as feed.

\subsubsection{Single Cascade}

Rather than the default value of $3.5 \%$ enrichment, we specify a top product of $75 \%$. In addition, we also specify 1,000 machines on the advanced parameters tab. The resulting cascade is depicted in Figure 26 and contains over 43 stages in the enriching section. More than $99 \%$ of the flow is out the tails, as the product flow rate is quite small. Note the ${ }^{238} \mathrm{U}$ assay in the product stream has been reduced to only $1.5 \%$ and cannot be reduced much further without incurring an error. This effect precludes enrichment to a product assay much higher than $75 \%$ and is discussed in more detail in Section 3.3.1. Very few machines are involved in the higher stages, as illustrated by Figure 27.

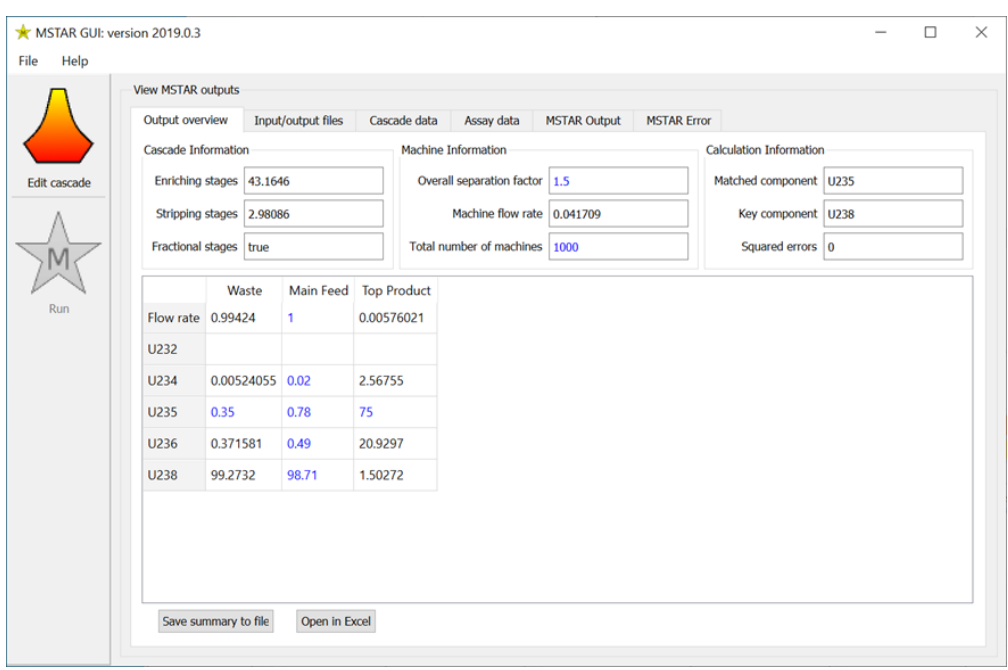

Figure 26. Highly enriched uranium overview.

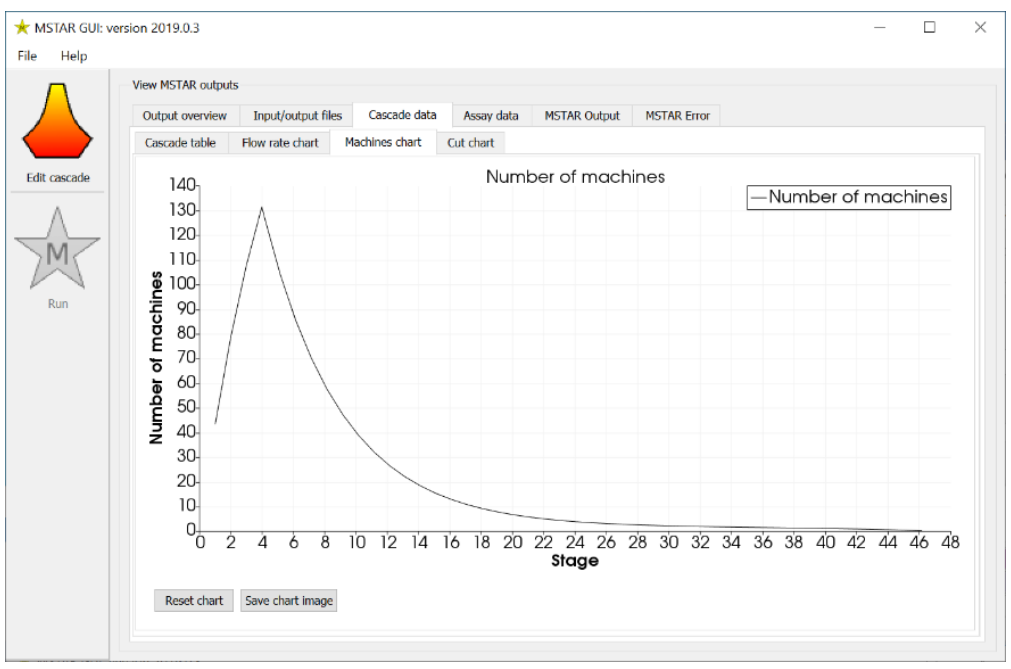

Figure 27. Machine chart for producing highly enriched uranium. 


\subsubsection{Batch Recycle}

We again consider input of recycled uranium as feed and enrich to $3.5 \%$. However, we now take this standard (declared) product of $3.5 \%$ enrichment and use it as feed into another cascade identical to the first. The cascade input is obtained from Figure 17 (or Figure 19) and is shown in Figure 28. An identical cascade is created by specifying the stage numbers for feed and top product (also gleaned from Figure 17) rather than tails and product assays. Results are shown in Figure 29 and indicate that a product of nearly $14 \%$ enrichment is produced.

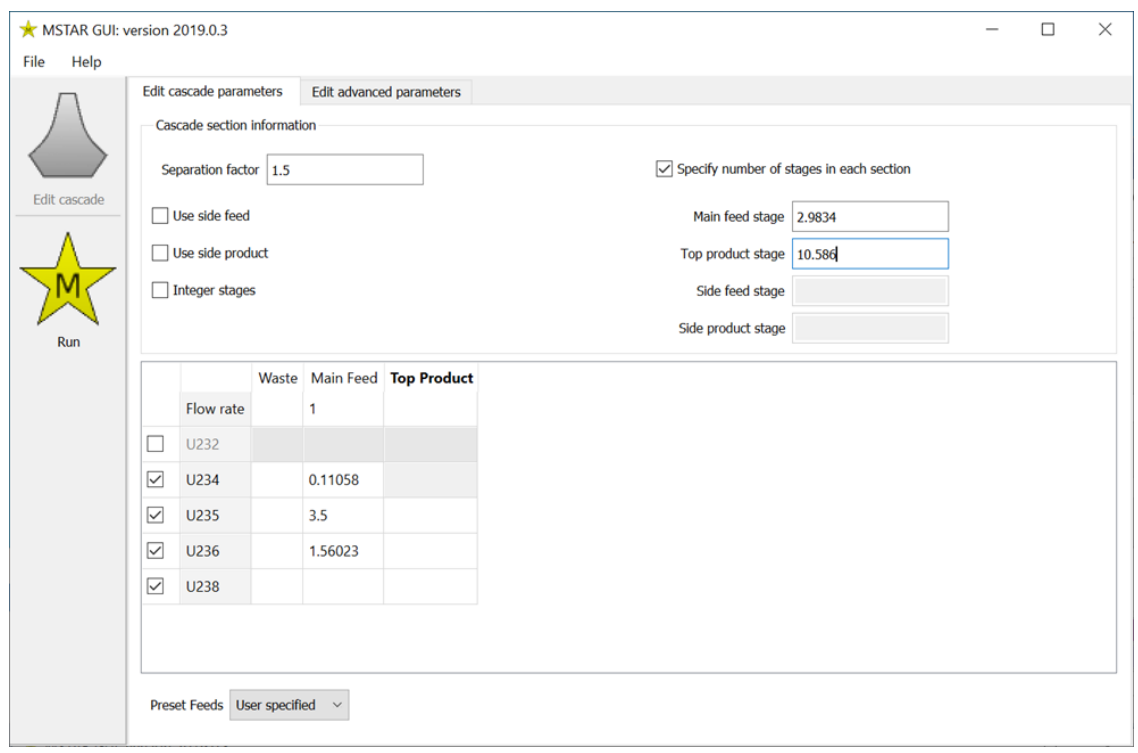

Figure 28. Input for batch recycle.

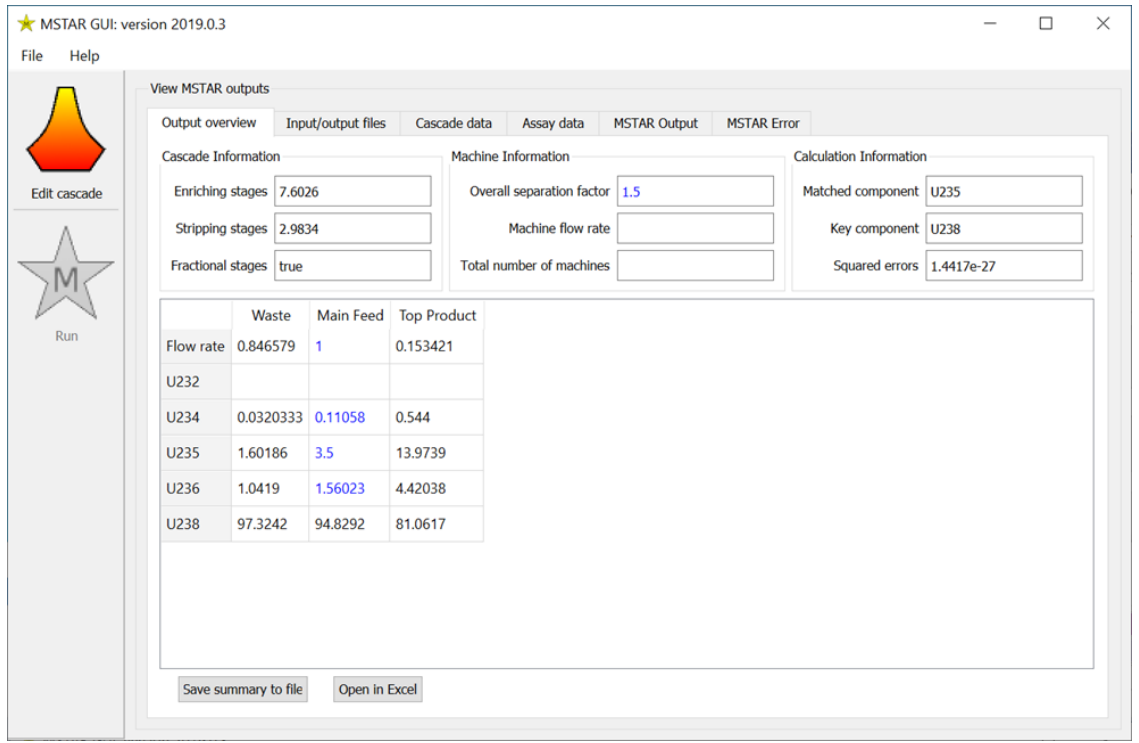

Figure 29. Results of batch recycle. 
If in the same way this $14 \%$ product is again used as feed to an identical cascade, the result is a product of almost $40 \%$ enrichment. A summary of the successive enrichments is provided in Error! Not a valid bookmark self-reference. If the product at $67 \%$ enrichment is used as feed in an attempt to produce very high enrichment, an error occurs. This is the same difficulty that was mentioned in Section 3.2.1, where enrichment beyond $75 \%{ }^{235} \mathrm{U}$ was problematic. Simulating this additional enrichment step is still possible, but special procedures are needed, as outlined in Section 3.3.1 and Appendix F.

Table 4. Batch recycle to obtain highly enriched uranium from reprocessed uranium.

\begin{tabular}{|c|c|c|c|}
\hline \multirow{2}{*}{ Feed description } & \multicolumn{3}{|c|}{ Product Assays (\%) } \\
\hline & ${ }^{234} \mathbf{U}$ & ${ }^{235} \mathbf{U}$ & ${ }^{236} \mathbf{U}$ \\
\hline 1. Initial recycled $U$ & 0.11058 & 3.5 & 1.56023 \\
\hline 2. Product from step 1 & 0.544 & 13.9739 & 4.42038 \\
\hline 3. Product from step 2 & 1.90771 & 39.7702 & 8.92732 \\
\hline 4. Product from step 3 & 3.98201 & 67.3713 & 10.7315 \\
\hline 5. Product from step 4 & \multicolumn{3}{|c|}{ Error incurred-key isotope too small } \\
\hline
\end{tabular}

\subsection{CASES THAT DO NOT WORK}

This section describes a few cases that are impossible to achieve physically or for which the MARC model cannot produce a result without special treatment.

\subsubsection{Recycled Fuel to Very High Enrichment}

This discussion is a follow-up to the limitations encountered in Section 3.2. From Figure 26, we note that the assay of ${ }^{238} \mathrm{U}$ in the product stream is very low, and if further enrichment is attempted, this inventory will dwindle to near zero. This complication is notably present in production of HEU from recycled U, since both ${ }^{235} \mathrm{U}$ and ${ }^{236} \mathrm{U}$ are being enriched. In a real cascade, such enrichment could be allowed to happen, but in the MARC formulation, the ${ }^{238} \mathrm{U}$ is usually the key isotope. Enrichment of other isotopes is measured by their ratio to the key isotope. It then becomes problematic for a quantity in the denominator of a ratio to approach zero, and the algorithm breaks down. The code will give an error if this is attempted. This problem can be alleviated by changing the key isotope from ${ }^{238} \mathrm{U}$ to ${ }^{236} \mathrm{U}$, a process that is described in APPENDIX F. 


\subsubsection{Side Product Too Large}

If we run the standard case of recycled uranium, the product flow rate is $13.6 \%$ of the feed flow, with the remainder exiting the cascade through the tails (see Figure 30). If we add a side product with ${ }^{235} \mathrm{U}$ assay of $2 \%$ and a flow of 0.1 (i.e., $10 \%$ of the feed flow), the top product flow is reduced to $8.4 \%$ of the feed flow. If the side product flow is increased to 0.2 , the top product is further reduced. If the side product is increased to 0.26 , the top product is barely a trickle, and if the side product is increased still further, the top product stops altogether or becomes negative. A negative value indicates that the top product actually needs to become a feed stream to provide the outflow for the side product. At this point, the code outputs an error.

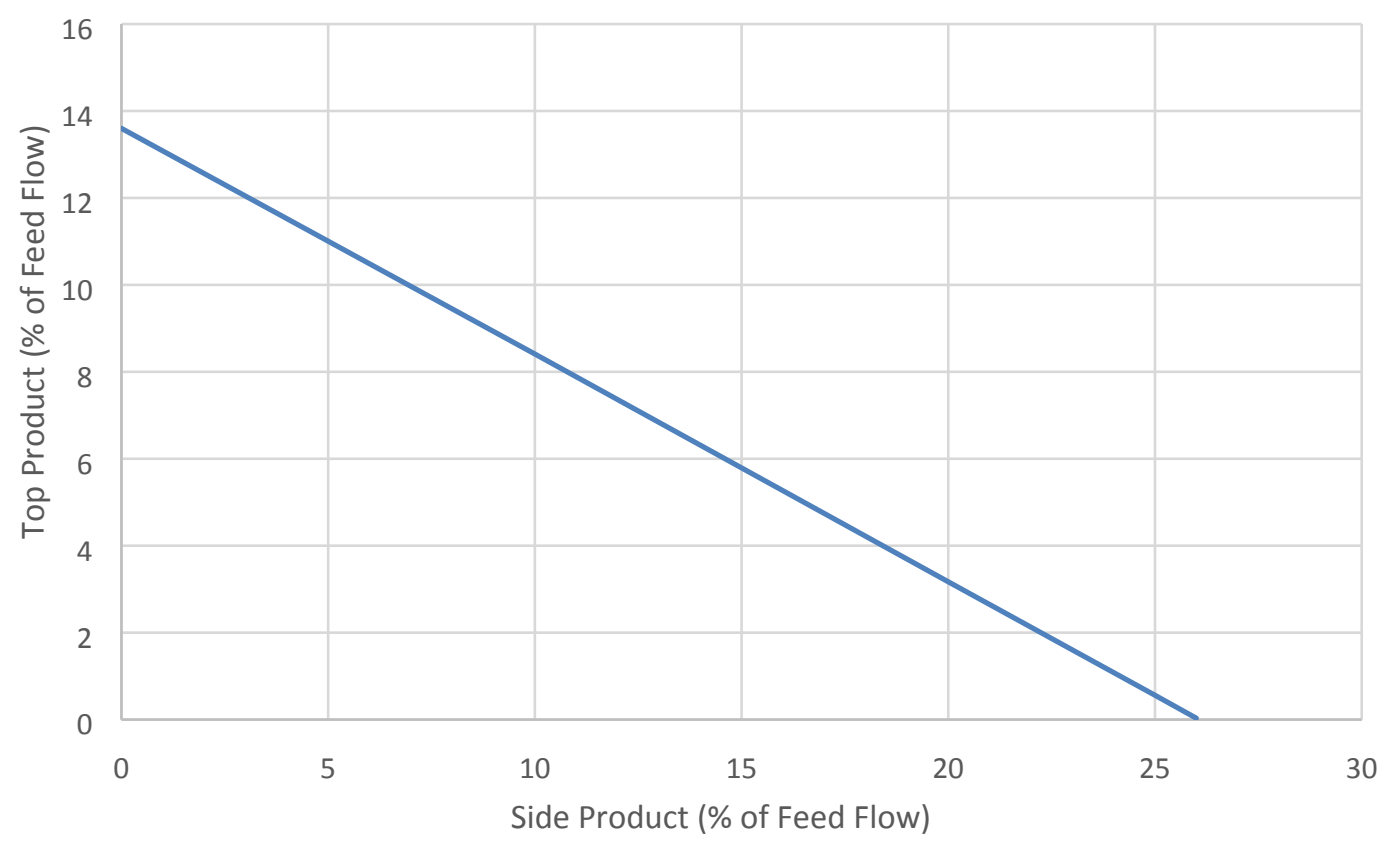

Figure 30. Side product effect on top product. 


\subsubsection{Side Feed Assay Too Small}

This case is similar to that of Section 3.1.2 for main feed of natural uranium and a side feed. Previously a side feed with assay $2 \%$ was used (Figure 20) and produced good results. If we lower the assay of the side feed, then its location in the cascade will decrease. Imposing a side feed of depleted uranium with ${ }^{235} \mathrm{U}$ assay of $0.3 \%$ and $0.001 \%{ }^{234} \mathrm{U}$ results in an error, as shown in Figure 31 and Figure 32, and serves to illustrate the constraints of this model. It is unrealistic that a feed stream even more depleted than the tails stream would be fed into a cascade because this would require more separative work and lower production than if the side feed were not even present. If we raise the side feed assay, we continue to see an error message until the ${ }^{235} \mathrm{U}$ assay exceeds $0.525 \%$. The reason is that the feed assay for stage 2 with no side streams is $0.525 \%$ (see Figure 19), and because this is the lowest stage at which a side feed could occur, this assay is the lower limit for a side feed to the cascade.

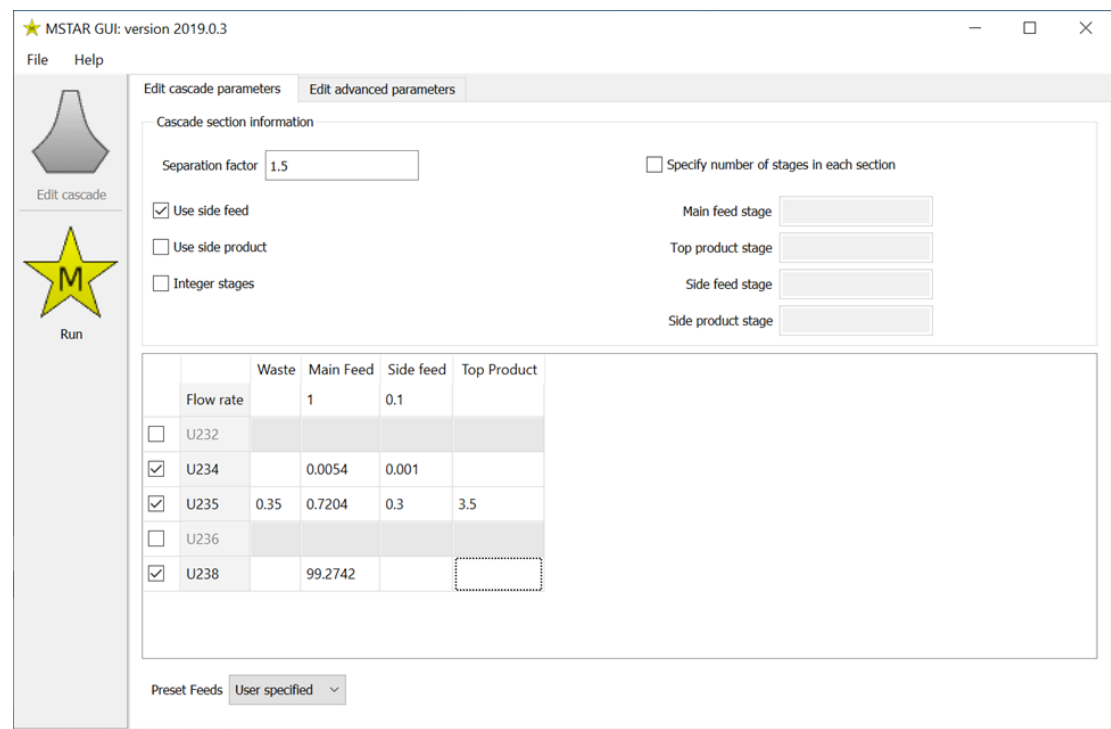

Figure 31. Low side feed assay input.

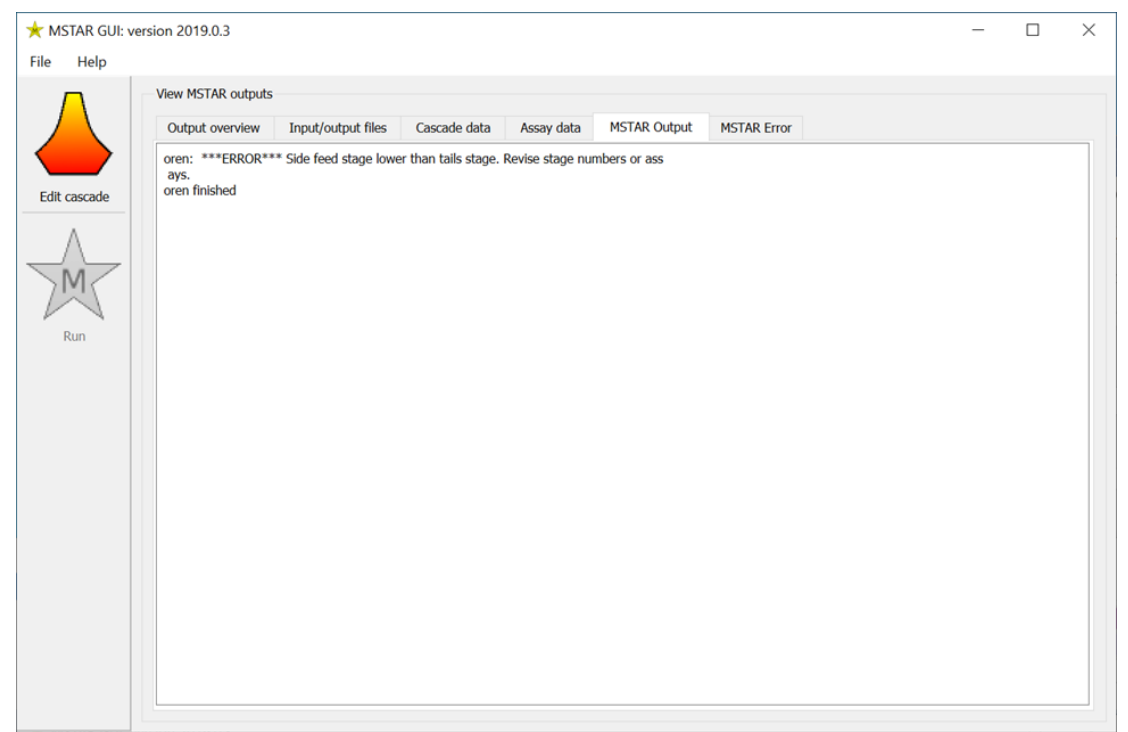

Figure 32. Error for low side feed assay. 


\section{GENERAL DESCRIPTION OF MODEL}

Having discussed the basic usage of the code, we now explore the fundamental mathematical development behind it. Much of the development in this section is obtained from unpublished notes of Ed Von Halle [5]. Some of his derivations have been modified so they are expressed in a form that may be easier for most readers to understand. In addition, some minor errors have been corrected, and a more thorough explanation of the equations is given.

\subsection{EXTERNAL VARIABLES}

Shown in Figure 33 are the principal regions and external variables in an enrichment cascade with side streams. The following quantities are associated with external streams and the stages where they enter or exit the cascade:

$X_{i P}, X_{i G}, X_{i F}, X_{i E}$, and $X_{i W}=$ assays in external streams: product, side product, feed, side feed, and waste for isotope $i$

$N_{P}, N_{G}, N_{F}$, and $N_{E}=$ stage numbers for product, side product, feed, and side feed (waste stream is drawn from Stage 1)

$P, G, F, E, W=$ flow rates for the various streams, all in consistent units

The flow rates represent mass or molar flows of $\mathrm{UF}_{6}$ and can be given in any useful unit such as kilograms per year $(\mathrm{kg} / \mathrm{y})$, milligrams per second $(\mathrm{mg} / \mathrm{s})$, or moles per hour $(\mathrm{mol} / \mathrm{h})$. The interchangeability of mass and molar flow units may seem incongruous on the surface. The internal calculations are based on gas dynamics and therefore use molar balances. Enrichment capability is usually related to mass of natural uranium feed, an easily measured quantity; hence, mass flow terms are often preferred for practicality. The molecular weights of $\mathrm{UF}_{6}$ for the two principal isotopes are 349 and 352 (for ${ }^{235} U$ and ${ }^{238} U$, respectively). The relative difference in these values is small enough that mass and mole fractions are nearly identical and can be interchanged with little impact on the accuracy (or utility) of calculations. 
As depicted in Figure 33, all flow rates are shown as leaving the cascade; hence, feed rates $(F$ and $E)$ need to be negative numbers for calculational purposes. Note also that the distinction between side and main feeds is somewhat academic. Both are feed streams, and we usually consider the main feed to be the largest. The ordering above suggests that the side feed is located below the main feed, but it could just as well occur above it. Note the feed streams actually enter the cascade at the next higher stage. Thus, under conventional parlance, the main feed stage is $N_{F}+1$, and the side feed stage is $N_{E}+1$.

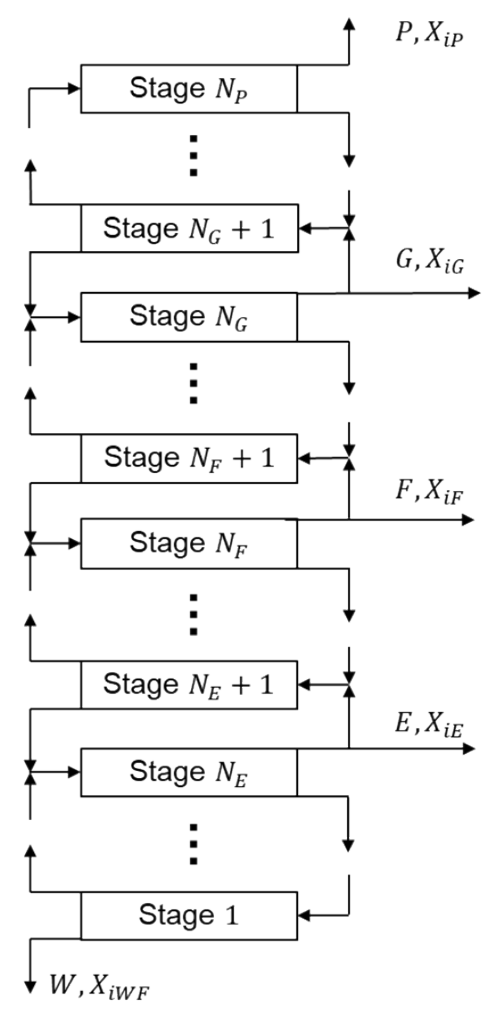

Figure 33. Cascade sections and external variables.

The quantities in Figure 33 completely define the cascade operation. They are interrelated by equations to be derived, and once some of them are supplied by the user, the others can be calculated. Because there is no material accumulation or loss within the system, the overall balance equations for flows and assays must be satisfied by

$$
\begin{gathered}
P+G+F+E+W=0 \\
P X_{i P}+G X_{i G}+F X_{i F}+E X_{i E}+W X_{i W}=0, i=1, \ldots, I .
\end{gathered}
$$

Furthermore, because they are defined as mole or mass fractions, assays are normalized according to the equations:

$$
\sum_{i=1}^{I} X_{i P}=\sum_{i=1}^{I} X_{i G}=\sum_{i=1}^{I} X_{i F}=\sum_{i=1}^{I} X_{i E}=\sum_{i=1}^{I} X_{i W}=1
$$

Note these equations are not independent because summing Eq. (2b) over all isotopes and applying Eq. (3) yields Eq. (2a). 


\subsection{INTERNAL CASCADE OPERATIONS}

Having identified those external variables which govern overall cascade operation, we now investigate the internal workings and variables that describe stagewise detail. With reference to Figure 34, we begin by defining the isotopic assays and overall flow rates in feed, product, and waste streams for each stage:

$z_{i n}, y_{i n}, x_{i n}=$ assay in feed, product and waste streams of stage $n$ for isotope $i$

$L_{n}, L_{n}^{\prime}, L_{n}^{\prime \prime}=$ feed, product, and waste flow rates for stage $n$

$\Lambda_{i n}, \Lambda_{i n}^{\prime}, \Lambda_{i n}^{\prime \prime}=$ feed, product, and waste isotopic flow rates for isotope $i$ at stage $n$

$T_{n}, \tau_{i n}=$ net upward flow rate and assay of isotope $i$ above stage $n$

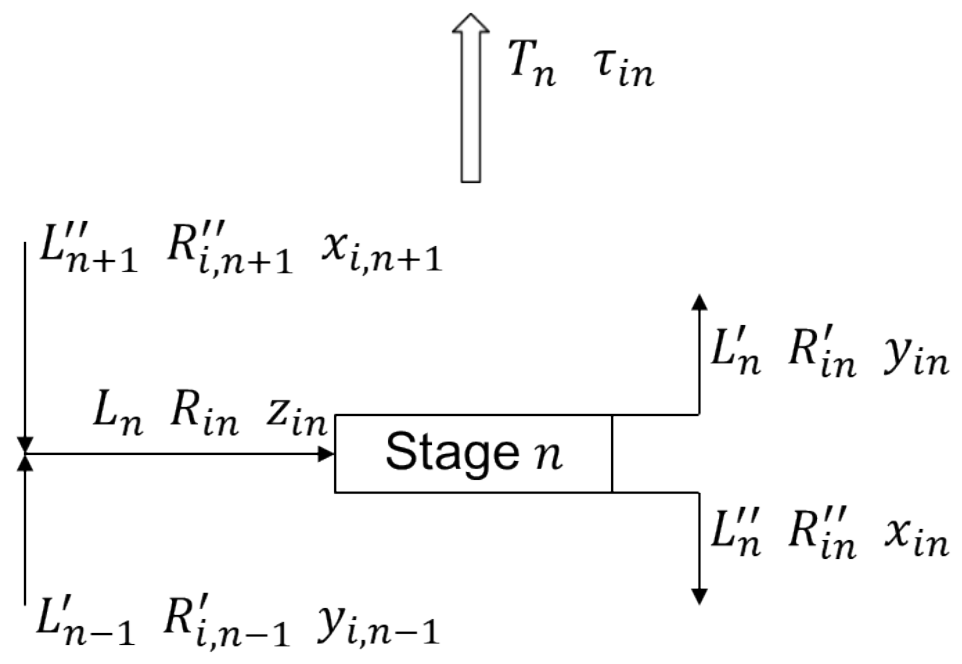

Figure 34. Stagewise variables

Note from these definitions that

$$
\Lambda_{\text {in }}=L_{n} z_{\text {in }} \quad \Lambda_{\text {in }}^{\prime}=L_{n}^{\prime} y_{\text {in }} \quad \Lambda_{\text {in }}^{\prime \prime}=L_{n}^{\prime \prime} x_{i n} .
$$

From simple material balances on flows and isotopes, we see that

$$
T_{n}=L_{n}^{\prime}-L_{n+1}^{\prime \prime}
$$

and

$$
\tau_{i n}=L_{n}^{\prime} y_{i n}-L_{n+1}^{\prime \prime} x_{i, n+1}=\Lambda_{i n}^{\prime}-\Lambda_{i, n+1}^{\prime \prime}
$$

All separations involve relative concentrations of one (or more) isotopes compared to another. Hence, we define one isotope to be the key isotope, the one against which all others are assessed (usually this is ${ }^{238} \mathrm{U}$ ) and designate it with the index $k$. Neglecting the stage index for now, the abundance ratio is the ratio of any other isotope to the key component:

$$
R_{i}=\frac{z_{i}}{z_{k}}, \quad R_{i}^{\prime}=\frac{y_{i}}{y_{k}}, \quad R_{i}^{\prime \prime}=\frac{x_{i}}{x_{k}}, \quad i=1,2, \ldots, I .
$$


We recognize that all assays must sum to unity, which implies

$$
\sum R_{i}=\frac{1}{z_{k}}, \quad z_{i}=z_{k} R_{i}=\frac{R_{i}}{\sum R_{j}^{\prime}} \quad i=1,2, \ldots, I .
$$

Note that analogous relations would hold for $R_{i}^{\prime}$ and $R_{i}^{\prime \prime}$. The overall stage separation factor is defined as a ratio of abundance ratios - the product ratio divided by the waste ratio:

$$
\alpha_{i}=\frac{R_{i}^{\prime}}{R_{i}^{\prime \prime}}=\frac{y_{i} / y_{k}}{x_{i} / x_{k}}, \quad i=1,2, \ldots, I .
$$

For simplicity, and because it is a very good approximation, we assume that the stage separation factors are independent of stage.

We also note from Eq. (7) that

$$
y_{i}=\alpha_{i} x_{i} \frac{y_{k}}{x_{k}} \Rightarrow \sum_{i=1}^{I} y_{i}=1=\frac{y_{k}}{x_{k}} \sum_{i=1}^{I} \alpha_{i} x_{i} \Rightarrow \frac{y_{k}}{x_{k}}=\frac{1}{\sum_{i=1}^{I} \alpha_{i} x_{i}} \Rightarrow y_{i}=\frac{\alpha_{i} x_{i}}{\sum_{j=1}^{I} \alpha_{j} x_{j}} .
$$

As mentioned in the introduction, the matched abundance ratio cascade (MARC) imposes an assumption in which we minimize mixing losses for a principal component, usually ${ }^{235} \mathrm{U}$. As seen in Figure 1, the feed to a stage is composed of the downflowing (waste) stream from the next higher stage and the upflowing (product) stream from the next lower stage. For MARC, we assume the assays in these streams are equal for one isotope (the matched component). Denoting this matched component with the index $m$, we have

$$
R_{m, n-1}^{\prime}=R_{m, n+1}^{\prime \prime} \quad \text { or } \quad R_{m, n}^{\prime}=R_{m, n+2}^{\prime \prime}
$$

which can be combined with Eq. (7) to get

$$
R_{m, n+2}^{\prime \prime}=\alpha_{m} R_{m n}^{\prime \prime}=\alpha_{m}^{2} R_{m, n-2}^{\prime \prime}=\ldots=\alpha_{m}^{n / 2} R_{m 2}^{\prime \prime}=\beta_{m}^{n} R_{m 2}^{\prime \prime} \text {, }
$$

where $\alpha_{m}=\beta_{m}^{2}$.

Now, combining Eqs. (5a), (5b), and (8) produces

$$
L_{n+1}^{\prime \prime} x_{i, n+1}+\tau_{i n}=L_{n}^{\prime} y_{i n}=\left(L_{n+1}^{\prime \prime}+T_{n}\right) y_{i n}=\left(L_{n+1}^{\prime \prime}+T_{n}\right) \frac{\alpha_{i} x_{i n}}{\sum_{j=1}^{I} \alpha_{j} x_{j n}} .
$$


We now define the stagewise isotopic flow rates $\Lambda_{i n}^{\prime \prime}=L_{n}^{\prime \prime} x_{i n}$ and substitute into Eq. (10), which yields

$$
\Lambda_{i, n+1}^{\prime \prime}-\left[\frac{L_{n+1}^{\prime \prime}+T_{n}}{L_{n}^{\prime \prime} \sum_{j=1}^{I} \alpha_{j} x_{j, n}}\right] \alpha_{i} \Lambda_{i, n}^{\prime \prime}=-\tau_{i n} .
$$

We note that the quantity in brackets is independent of isotope and may be independent of stage as well. We show in APPENDIX A that this quantity is equal to $\beta_{m}^{-1}$ for all values of $n$, which allows Eq. (11) to be written as

$$
\Lambda_{i, n+1}^{\prime \prime}-\alpha_{i}^{*} \Lambda_{i, n}^{\prime \prime}=-\tau_{i n}
$$

where

$$
\alpha_{i}^{*} \equiv \alpha_{i} / \beta_{m}
$$

Combining Eqs. (5b) and (12a) yields

$$
\Lambda_{i, n}^{\prime}=\alpha_{i}^{*} \Lambda_{i, n}^{\prime \prime}
$$

We now use Eq. (12a) repeatedly, so that for any two stages in a common section of the cascade (where $\tau_{i n}=\tau_{i}$ is the same for every stage):

$$
\begin{gathered}
\Lambda_{i n}^{\prime \prime}=\alpha_{i}^{*} \Lambda_{i, n-1}^{\prime \prime}-\tau_{i}=\alpha_{i}^{*}\left(\alpha_{i}^{*} \Lambda_{i, n-2}^{\prime \prime}-\tau_{i}\right)-\tau_{i}=\left(\alpha_{i}^{*}\right)^{2} \Lambda_{i, n-2}^{\prime \prime}-\tau_{i}\left(1+\alpha_{i}^{*}\right) \\
=\left(\alpha_{i}^{*}\right)^{2}\left(\alpha_{i}^{*} \Lambda_{i, n-3}^{\prime \prime}-\tau_{i}\right)-\tau_{i}\left(1+\alpha_{i}^{*}\right)=\left(\alpha_{i}^{*}\right)^{3} \Lambda_{i, n-3}^{\prime \prime}-\tau_{i}\left[1+\alpha_{i}^{*}+\left(\alpha_{i}^{*}\right)^{2}\right] \\
=\ldots=\left(\alpha_{i}^{*}\right)^{n-j} \Lambda_{i j}^{\prime \prime}-\tau_{i}\left[1+\alpha_{i}^{*}+\left(\alpha_{i}^{*}\right)^{2} \ldots+\left(\alpha_{i}^{*}\right)^{n-j-1}\right] \\
=\left(\alpha_{i}^{*}\right)^{n-j} \Lambda_{i j}^{\prime \prime}-\tau_{i} \frac{1-\left(\alpha_{i}^{*}\right)^{n-j}}{1-\alpha_{i}^{*}}
\end{gathered}
$$

Note that Eq. (14) assumes that $\alpha_{i}^{*} \neq 1$, which is usually true. The special case of $\alpha_{i}^{*}=1$ is discussed in Section 4.8, where alternate equations are derived. This relation holds for any section of the cascade where $\tau_{i}$ is constant and does not change with stage number. It is the fundamental equation describing stagewise progression of flow. 


\subsection{JUNCTIONS BETWEEN CASCADE SECTIONS}

We now consider the stages immediately above and below external streams of the cascade as shown in Figure 35 for the junction between the top product section and the side product section. We attach a superscript in parentheses to denote the section in which a flow is considered. Balance equations for this junction are

$$
\Lambda_{i, N_{G}}^{\prime(P)}=\Lambda_{i, N_{G}}^{\prime \prime}(P)+\tau_{l}^{(P)}=\Lambda_{i, N_{G}}^{\prime \prime}(P)+P X_{i P}
$$

and

$$
\Lambda_{i, N_{G}}^{\prime(G)}=\Lambda_{i, N_{G}+1}^{\prime \prime(G)}+\tau_{i}^{(G)}=\Lambda_{i, N_{G}+1}^{\prime \prime(G)}+P X_{i P}+G X_{i G}
$$

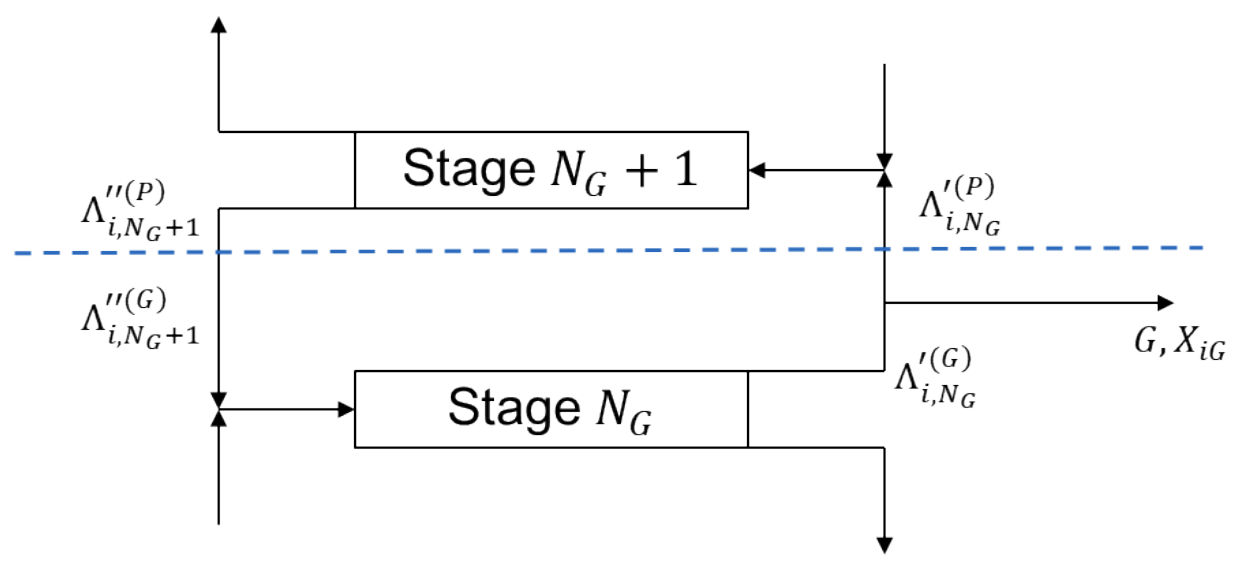

Figure 35. Junction between top two sections.

Here, we recognize $\Lambda_{i, N_{G}+1}^{\prime \prime}(P)$ as the downflowing stream that leaves stage $N_{G}+1$, and $\Lambda_{i, N_{G}+1}^{\prime \prime}(G)$ is the downflowing stream that forms part of the feed into stage $N_{G}$. Likewise, $\Lambda_{i, N_{G}}^{\prime(G)}$ is the upflowing stream that leaves stage $N_{G}$, and $\Lambda_{i, N_{G}}^{\prime(P)}$ is the upflowing stream that contributes to the feed into stage $N_{G}+1$. From Figure 35, it appears that appropriate balance equations at the junction (the dashed line) are as follows:

$$
\Lambda_{i, N_{G}}^{\prime \prime(P)}=\Lambda_{i, N_{G}}^{\prime \prime(G)} \quad \text { and } \quad \Lambda_{i, N_{G}}^{\prime(G)}=\Lambda_{i, N_{G}}^{\prime(P)}+G X_{i G}
$$

Note that Eq. (16a) is slightly different from the junction treatment of Von Halle [5], which is discussed further in APPENDIX B. For the junction points of other cascade sections, the analogues for Eq. (16a) are

$$
\Lambda_{i, N_{F}+1}^{\prime \prime}(G)=\Lambda_{i, N_{F}}^{\prime \prime}(F)+1 \quad \text { and } \quad \Lambda_{i, N_{F}}^{\prime(F)}=\Lambda_{i, N_{F}}^{\prime(G)}+F X_{i F},
$$

and

$$
\Lambda_{i, N_{E}}^{\prime \prime}(F)+1=\Lambda_{i, N_{E}}^{\prime \prime(E)}+1 \quad \text { and } \quad \Lambda_{i, N_{E}}^{\prime(E)}=\Lambda_{i, N_{E}}^{\prime(F)}+E X_{i E}
$$




\subsection{SECTIONAL CASCADE EQUATIONS}

We now continue to follow the general treatment of Von Halle in developing the governing equations for each section of the cascade. The principal equation is Eq. (14), which is applied within each section.

\subsubsection{Top Section}

This section is located between side product and top product (i.e., for stages between $N_{G}+1$ and $N_{P}$ [inclusive]). In this section $\tau_{i}=P X_{i P}$. Using Eq. (14) with $n=N_{P}+1$ and $j=N_{G}+1$ in Eq. (14) gives

$$
\Lambda_{i, N_{P}+1}^{\prime \prime}\left(N_{)}=0=\left(\alpha_{i}^{*}\right)^{N_{P}-N_{G}} \Lambda_{i, N_{G}}^{\prime \prime}\left(N_{)}\right)+P X_{i P}\left[\frac{1-\left(\alpha_{i}^{*}\right)^{N_{P}-N_{G}}}{1-\alpha_{i}^{*}}\right]\right.
$$

which is rearranged to get

$$
\left(\alpha_{i}^{*}\right)^{-N_{G}} \Lambda_{i, N_{G}^{\prime \prime}}^{\prime \prime}(P)=P X_{i P}\left[\frac{\left(\alpha_{i}^{*}\right)^{-N_{G}}-\left(\alpha_{i}^{*}\right)^{-N_{P}}}{\alpha_{i}^{*}-1}\right] .
$$

Utilizing Eq. (13), we also have

$$
\left(\alpha_{i}^{*}\right)^{-N_{G}} \Lambda_{i, N_{G}}^{\prime(P)}=P X_{i P} \alpha_{i}^{*}\left[\frac{\left(\alpha_{i}^{*}\right)^{-N_{G}}-\left(\alpha_{i}^{*}\right)^{-N_{P}}}{\alpha_{i}^{*}-1}\right] .
$$

\subsubsection{Section $G$}

This section represents stages between the feed and side product (i.e., between stages $N_{F}+1$ and $N_{G}$ [inclusive]), where $\tau_{i}=P X_{i P}+G X_{i G}$. Substituting $n=N_{G}+1$ and $j=N_{F}+1$ into Eq. (14) gives

$$
\Lambda_{i, N_{G}+1}^{\prime \prime(G)}=\left(\alpha_{i}^{*}\right)^{N_{G}-N_{F}} \Lambda_{i, N_{F}^{\prime \prime}+1}^{\prime \prime}(G)\left(P X_{i P}+G X_{i G}\right)\left[\frac{1-\left(\alpha_{i}^{*}\right)^{N_{G}-N_{F}}}{1-\alpha_{i}^{*}}\right]
$$

which can be rearranged to get

$$
\left(\alpha_{i}^{*}\right)^{-N_{f} \Lambda_{i, N_{F}+1}^{\prime \prime}(G)}-\left(\alpha_{i}^{*}\right)^{-N_{G}} \Lambda_{i, N_{G}^{\prime \prime}(G)}=\left(P X_{i P}+G X_{i G}\right)\left[\frac{\left(\alpha_{i}^{*}\right)^{-N_{F}}-\left(\alpha_{i}^{*}\right)^{-N_{G}}}{\alpha_{i}^{*}-1}\right] .
$$

Again using Eq. (13), we have

$$
\left(\alpha_{i}^{*}\right)^{-N_{F}} \Lambda_{i, N_{F}}^{\prime(G)}-\left(\alpha_{i}^{*}\right)^{-N_{G}} \Lambda_{i, N_{G}^{\prime}(G)}=\left(P X_{i P}+G X_{i G}\right) \alpha_{i}^{*}\left[\frac{\left(\alpha_{i}^{*}\right)^{-N_{F}}-\left(\alpha_{i}^{*}\right)^{-N_{G}}}{\alpha_{i}^{*}-1}\right] .
$$




\subsubsection{Section F}

This section represents stages between the two external feed stages (i.e., stages between $N_{E}+1$ and $N_{F}$ [inclusive]). This section is completely analogous to Section $\mathrm{G}$, except for $\tau_{i}=P X_{i P}+G X_{i G}+F X_{i F}$. Hence, we will simply record the resulting equations without derivation:

$$
\begin{gathered}
\left(\alpha_{i}^{*}\right)^{-N_{E}} \Lambda_{i, N_{E}^{\prime \prime}(F)+1}-\left(\alpha_{i}^{*}\right)^{-N_{F}} \Lambda_{i, N_{F}^{\prime \prime}(F)+1}^{\prime(F)}=\left(P X_{i P}+G X_{i G}+F X_{i F}\right)\left[\frac{\left(\alpha_{i}^{*}\right)^{-N_{E}}-\left(\alpha_{i}^{*}\right)^{-N_{F}}}{\alpha_{i}^{*}-1}\right] \\
\left(\alpha_{i}^{*}\right)^{-N_{E}} \Lambda_{i, N_{E}}^{\prime(F)}-\left(\alpha_{i}^{*}\right)^{-N_{F}} \Lambda_{i, N_{F}^{\prime}}^{\prime(F)}=\left(P X_{i P}+G X_{i G}+F X_{i F}\right) \alpha_{i}^{*}\left[\frac{\left(\alpha_{i}^{*}\right)^{-N_{E}}-\left(\alpha_{i}^{*}\right)^{-N_{F}}}{\alpha_{i}^{*}-1}\right]
\end{gathered}
$$

\subsubsection{Section E}

Section $\mathrm{E}$ is the lowest section between stage 1 and stage $N_{E}$ (inclusive), where $\tau_{i}=P X_{i P}+G X_{i G}+F X_{i F}$ $+E X_{i E}$. Using $n=N_{E}+1$ and $j=1$ in Eq. (14) gives

$$
\Lambda_{i, N_{E}}^{\prime \prime(E)}+\left(\alpha_{i}^{*}\right)^{N_{E}} \Lambda_{i 1}^{\prime \prime(E)}-\left(P X_{i P}+G X_{i G}+F X_{i F}+E X_{i E}\right)\left[\frac{1-\left(\alpha_{i}^{*}\right)^{N_{E}}}{1-\alpha_{i}^{*}}\right]
$$

Now, $\Lambda_{i 1}^{\prime \prime}(E)$ is the downflow of isotope $i$ from the bottom stage, which is also the waste flow from the entire cascade; hence, $\Lambda_{i 1}^{\prime \prime(E)}=W X_{i W}$. The overall isotopic balance of Eq. (2) gives

$$
\Lambda_{i 1}^{\prime \prime(E)}=W X_{i W}=-\left(P X_{i P}+G X_{i G}+F X_{i F}+E X_{i E}\right)=-\tau_{i}^{(E)}
$$

Substituting Eq. (20a) into Eq. (20) and rearranging gives

$$
-\left(\alpha_{i}^{*}\right)^{-N_{E}} \Lambda_{i, N_{E}}^{\prime \prime}(E)+1=\tau_{i}^{(E)}+\tau_{i}^{(E)}\left[\frac{\left(\alpha_{i}^{*}\right)^{-N_{E}}-1}{1-\alpha_{i}^{*}}\right]=\left(P X_{i P}+G X_{i G}+F X_{i F}+E X_{i E}\right)\left[\frac{\alpha_{i}^{*}-\left(\alpha_{i}^{*}\right)}{\alpha_{i}^{*}-1}\right.
$$

Using Eq. (21a) together with Eq. (13), we have

$$
\left(\alpha_{i}^{*}\right)^{-N_{E}} \Lambda_{i, N_{e}}^{\prime(E)}=\alpha_{i}^{*}\left(P X_{i P}+G X_{i G}+F X_{i F}+E X_{i E}\right)\left[\frac{\left(\alpha_{i}^{*}\right)^{-N_{E}}-1}{\alpha_{i}^{*}-1}\right]
$$




\subsection{GENERAL CASCADE EQUATION}

The sectional equations in the previous section express the cascade properties in terms of the internal flow rates as well as the external streams. We wish to establish relationships between the external streams that do not involve internal stagewise variables. To do this, we add Eqs. (17a), (18a), (19a), and (21a). Alternatively, we could add Eqs. (17b), (18b), (19b), and (21b), which would provide the same result. Adding the left sides of Eqs. (17a), (18a), (19a), and (21a) gives

$$
\begin{aligned}
& \left(\alpha_{i}^{*}\right)^{-N_{G}} \Lambda_{i, N_{G}+1}^{\prime \prime(P)}+\left(\alpha_{i}^{*}\right)^{-N_{F}} \Lambda_{i, N_{F}+1}^{\prime \prime(G)}-\left(\alpha_{i}^{*}\right)^{-N_{G}} \Lambda_{i, N_{G}}^{\prime \prime(G)}+1+\left(\alpha_{i}^{*}\right)^{-N_{E}} \Lambda_{i, N_{E}}^{\prime \prime}(F)+\left(\alpha_{i}^{*}\right)^{-N_{F}} \Lambda_{i, N}^{\prime \prime \prime} \\
& -\left(\alpha_{i}^{*}\right)^{-N_{E}} \Lambda_{i, N_{E}+1}^{\prime \prime}(E) \text {. }
\end{aligned}
$$

Adding the right sides of the same equations and simplifying gives

$$
\begin{aligned}
& P X_{i P}\left[\frac{\left(\alpha_{i}^{*}\right)^{-N_{G}}-\left(\alpha_{i}^{*}\right)^{-N_{P}}}{\alpha_{i}^{*}-1}\right]+\left(P X_{i P}+G X_{i G}\right)\left[\frac{\left(\alpha_{i}^{*}\right)^{-N_{F}}-\left(\alpha_{i}^{*}\right)^{-N_{G}}}{\alpha_{i}^{*}-1}\right]+\left(P X_{i P}+G X_{i G}+F X_{i F}\right. \\
& \left.\frac{\left(\alpha_{i}^{*}\right)^{-N_{E}}-\left(\alpha_{i}^{*}\right)^{-N_{F}}}{\alpha_{i}^{*}-1}\right]+\left(P X_{i P}+G X_{i G}+F X_{i F}+E X_{i E}\right)\left[\frac{\alpha_{i}^{*}-\left(\alpha_{i}^{*}\right)^{-N_{E}}}{\alpha_{i}^{*}-1}\right] \\
& =\frac{1}{\alpha_{i}^{*}-1}\left\{P X_{i P}\left[\alpha_{i}^{*}-\left(\alpha_{i}^{*}\right)^{-N_{P}}\right]+G X_{i G}\left[\alpha_{i}^{*}-\left(\alpha_{i}^{*}\right)^{-N_{G}}\right]+F X_{i F}\left[\alpha_{i}^{*}-\left(\alpha_{i}^{*}\right)^{-l}\right.\right. \\
& \left.\left.\alpha_{i}^{*}-\left(\alpha_{i}^{*}\right)^{-N_{E}}\right]\right\} \text {. }
\end{aligned}
$$

Equating Eqs. (22a) and (22b) yields the equation

$$
\begin{gathered}
\frac{1}{\alpha_{i}^{*}-1}\left\{P X_{i P}\left[\alpha_{i}^{*}-\left(\alpha_{i}^{*}\right)^{-N_{P}}\right]+G X_{i G}\left[\alpha_{i}^{*}-\left(\alpha_{i}^{*}\right)^{-N_{G}}\right]+F X_{i F}\left[\alpha_{i}^{*}-\left(\alpha_{i}^{*}\right)^{-N_{F}}\right]+E X_{i E}\left[\alpha_{i}^{*}-\right.\right. \\
=0 .
\end{gathered}
$$

which can be simplified to get

$$
\begin{gathered}
P X_{i P}\left[1-\left(\alpha_{i}^{*}\right)^{-N_{P}-1}\right]+G X_{i G}\left[1-\left(\alpha_{i}^{*}\right)^{-N_{G}-1}\right]+F X_{i F}\left[1-\left(\alpha_{i}^{*}\right)^{-N_{F}-1}\right]+E X_{i E}\left[1-\left(\alpha_{i}^{*}\right)\right. \\
=0, \quad i=1, \ldots, I .
\end{gathered}
$$

Substituting Eq. (2) and rearranging produces the following alternative form, which is useful in practice:

$$
W X_{i W} \alpha_{i}^{*}+P X_{i P}\left(\alpha_{i}^{*}\right)^{-N_{P}}+G X_{i G}\left(\alpha_{i}^{*}\right)^{-N_{G}}+F X_{i F}\left(\alpha_{i}^{*}\right)^{-N_{F}}+E X_{i E}\left(\alpha_{i}^{*}\right)^{-N_{E}}=0
$$

Equation (23) is true for each isotope and involves only the external stream variables, numbers of stages, and the separation factors. We note that Eq. (23) is slightly different from the result that Von Halle obtained, as described in APPENDIX B. 


\subsection{SECTIONAL STAGE NUMBERS}

By itself, Eq. (23) cannot distinguish the numbers of stages within each section of the cascade. However, using the abundance ratio equations for the matched isotope, we have additional information to determine these numbers. Combining Eqs. (7) and (9) gives the relation

$$
R_{m n}^{\prime}=\beta_{m}^{n-j} R_{m j}^{\prime}
$$

which is true throughout the cascade, including at junction points where external streams are located. Applying Eq. (24) in the top section gives

$$
R_{m N_{P}}^{\prime}=\beta_{m}^{N_{P}-N_{G}} R_{m N_{G}}^{\prime}
$$

From Eqs. (1) and (9), we note that for the matched isotope, we have $\alpha=\alpha_{m}=\beta_{m}^{2}$, which implies that

$$
N_{P}-N_{G}=\frac{\ln \left(R_{m N_{P}}^{\prime} / R_{m, N_{G}}^{\prime}\right)}{\ln \left(\beta_{m}\right)}=\frac{2}{\ln (\alpha)} \ln \left[\frac{X_{m P / X_{k P}}}{X_{m G / X_{k G}}}\right] .
$$

Similar equations can be developed for the other points involving stage numbers and external streams:

$$
\begin{aligned}
& N_{G}-N_{F}=\frac{2}{\ln (\alpha)} \ln \left[\frac{X_{m G / X_{k G}}}{X_{m F / X_{k F}}}\right] \\
& N_{F}-N_{E}=\frac{2}{\ln (\alpha)} \ln \left[\frac{X_{m F / X_{k F}}}{X_{m E / X_{k E}}}\right]
\end{aligned}
$$

For the bottom section, we have

$$
R_{m N_{E}}^{\prime}=\beta_{m}^{N_{E}-1} R_{m 1}^{\prime}=\alpha \beta_{m}^{N_{E}-1} R_{m 1}^{\prime \prime}=\beta_{m}^{N_{E}+1} R_{m 1}^{\prime \prime}
$$

Consequently, for this section, we have the relation

$$
N_{E}=-1+\frac{2}{\ln (\alpha)} \ln \left[\frac{X_{m E} / X_{k E}}{X_{m W / X_{k W}}}\right] .
$$

We note that if there is no side feed, then Eqs. $(25 \mathrm{c}-\mathrm{d})$ are replaced by the single equation:

$$
N_{F}=-1+\frac{2}{\ln (\alpha)} \ln \left[\frac{X_{m F} / X_{k F}}{X_{m W / X_{k W}}}\right]
$$

and if there is no side product, then Eqs. $(25 \mathrm{a}-\mathrm{b})$ are replaced by the single equation 


$$
N_{P}-N_{F}=\frac{2}{\ln (\alpha)} \ln \left[\frac{X_{m P} / X_{k P}}{X_{m F} / X_{k F}}\right]
$$

\subsection{APPORTIONMENT OF PRODUCT STREAMS}

Additional information is needed to determine minor isotope ratios. From Eq. (17b) we have

$$
\Lambda_{i, N_{g}}^{\prime(P)}=P X_{i P} \alpha_{i}^{*}\left[\frac{1-\left(\alpha_{i}^{*}\right)^{N_{G}-N_{P}-1}}{\alpha_{i}^{*}-1}\right] .
$$

From the definition of the isotopic flows $\Lambda_{i, n}^{\prime}$, we can determine both the stagewise flows and assays:

$$
L_{n}^{\prime}=\sum_{i} \Lambda_{i n}^{\prime}, \quad y_{i n}=\frac{\Lambda_{i, n}^{\prime}}{L_{n}^{\prime}} .
$$

Combining Eqs. (26) and (27) yields

$$
X_{i G}=y_{i N_{G}}=\frac{\Lambda_{i, N_{G}}^{\prime}}{L_{N_{G}}^{\prime}}=\frac{X_{i P} \alpha_{i}^{*}\left[\frac{1-\left(\alpha_{i}^{*}\right)^{N_{G}-N_{P}-1}}{\alpha_{i}^{*}-1}\right]}{\sum_{j}\left\{X_{j P} \alpha_{j}^{*}\left[\frac{1-\left(\alpha_{j}^{*}\right)^{N_{G}-N_{P}-1}}{\alpha_{j}^{*}-1}\right]\right.} .
$$

\subsection{SINGULARITIES}

In some cases, it is possible that a value of $\alpha_{i}^{*}$ may be equal to unity, such as when the matched isotope is ${ }^{234} \mathrm{U}$ instead of ${ }^{235} \mathrm{U}$. If the key isotope is ${ }^{238} \mathrm{U}$ and reprocessed fuel is used, then for ${ }^{236} \mathrm{U}, \alpha_{i}^{*}=1$. This poses a substantial problem, since many of the derived equations in previous sections have the term $\alpha_{i}^{*}$ -1 in the denominator, among other things. In these situations, the singularities can be removed by taking limits of the equations as $\alpha_{i}^{*} \rightarrow 1$. The following modified equations reflect the treatment for this special situation:

$$
P X_{i P}\left(N_{P}+1\right)+G X_{i G}\left(N_{G}+1\right)+F X_{i F}\left(N_{F}+1\right)+E X_{i E}\left(N_{E}+1\right)=0,
$$

which can also be expressed as

$$
P X_{i P} N_{P}+G X_{i G} N_{G}+F X_{i F} N_{F}+E X_{i E} N_{E}-W X_{i W}=0 .
$$

Numerator of Eq. (28) $=X_{i P}\left(N_{P}-N_{G}+1\right)$. 
Similar considerations must be made elsewhere in this development and will be recorded. Note that for the matched component, we cannot have $\alpha_{m}^{*}=1$ because this would imply that $\alpha=1$, and no enrichment would occur. Hence, Eqs. (25a-f) are unaffected. 


\section{GENERAL SOLUTION PROCEDURE}

The complete list of external variables is shown in

Table 5. If these are known, then the cascade is completely defined. Some of these variables will be specified as input parameters, but others will be calculated using the equations developed in Section 2. Note although the derivation has tacitly assumed that numbers of stages are integer quantities, the final governing equations, especially Eqs. (25a-d), indicate that noninteger values will likely result. Because real enrichment plants do have integer numbers of stages, we provide an option in Section 3.1.3 for outputting integer numbers of stages. However, the basic solution process assumes that these quantities are real numbers, which means that fractional stages can (and generally do) result.

Table 5. Cascade variables.

\begin{tabular}{|c|c|c|}
\hline Index & Variable & Description \\
\hline 1 & $\alpha$ & Separation factor for matched component \\
\hline 2 & $N_{E}$ & Stage number for side feed \\
\hline 3 & $N_{F}$ & Stage number for main feed \\
\hline 4 & $N_{G}$ & Stage number for side product \\
\hline 5 & $N_{P}$ & Stage number for top product \\
\hline 6 & $W$ & Mass flow rate of tails (waste) stream \\
\hline 7 & $E$ & Mass flow rate of side feed stream \\
\hline 8 & $F$ & Mass flow rate of main feed stream \\
\hline 9 & $G$ & Mass flow rate of side product stream \\
\hline 10 & $P$ & Mass flow rate of top product stream \\
\hline $10+i$ & $X_{i W}$ & Waste assays \\
\hline $10+I+i$ & $X_{i E}$ & Side feed assays \\
\hline $10+2 I+i$ & $X_{i F}$ & Main feed assays \\
\hline $10+3 I+i$ & $X_{i G}$ & Side product assays \\
\hline $10+4 I+i$ & $X_{i P}$ & Top product assays \\
\hline
\end{tabular}

There is a total of $10+5 I$ unknowns if both side streams are included. The number of independent equations derived in Section 2 is $5+3 I$ as summarized in Table 6. We thus expect that inputs should total $5+2 I$.

Table 6. Summary of equations.

\begin{tabular}{|l|c|c|}
\hline \multicolumn{1}{|c|}{ Description } & Equation & Number \\
\hline Sectional stage numbers & $25 \mathrm{a}-\mathrm{d}$ & 4 \\
\hline Isotopic normalizations & $3 \mathrm{a}-\mathrm{e}$ & 5 \\
\hline Isotopic balances, $i \neq m, k$ & $2 \mathrm{~b}$ & $I-2$ \\
\hline Flow balance & $2 \mathrm{a}$ & 1 \\
\hline Cascade equations, $i \neq m, k$ & 23 & $I-2$ \\
\hline Product assay relations, $i \neq m$ & 28 & $I-1$ \\
\hline
\end{tabular}




\subsection{SOLUTION ALGORITHM}

Having derived a set of governing equations in Section 2, we now look to methods for solving them and fully characterizing an enrichment cascade. Because the equations are nonlinear, an iterative approach will be necessary.

\subsubsection{Von Halle Solution}

In his original formulation [5,6], Von Halle made strict input requirements, specifying that all of the input variables shown in Table 7 must be supplied by the user. The solution algorithm depends on knowledge of these values and will not attain a solution if they are not available. Summing the last column indicates that a total of $5+2 I$ inputs are required. Von Halle does allow top product flow and assays to be specified instead of feed flow and assay, but this is the only flexibility allowed.

Table 7. Input variables required by Von Halle.

\begin{tabular}{|l|c|c|}
\hline \multicolumn{1}{|c|}{ Description } & Variable & Number \\
\hline Flow rates for feed, side feed, and side product & $E, F, G$ & 3 \\
\hline All assays for feed and side feed & $X_{i F}, X_{i E}$ & $2(I-1)$ \\
\hline Assay of matched component in waste, product, side product & $X_{m W}, X_{m P}, X_{m G}$ & 3 \\
\hline Separation factor & $\alpha$ & 1 \\
\hline
\end{tabular}

If all the inputs from Table 7 are supplied exactly, we adopt the following algorithm, which bears some similarity to Von Halle's approach:

1) Determine remaining flow rates using Eqs. (2a) and (2b), evaluating the latter for the matched component $i=m$ :

$$
\begin{gathered}
P=\left[G\left(X_{m G}-X_{m W}\right)+F\left(X_{m F}-X_{m W}\right)+E\left(X_{m E}-X_{m W}\right)\right] /\left(X_{m P}-X_{m W}\right) . \\
W=-(P+G+F+E) .
\end{gathered}
$$

2) Approximate all minor isotope assays (anything that is neither the key nor matched component). A simple form is as a small fraction of the matched component:

$$
X_{i W}=X_{m W} * 0.01, X_{i G}=X_{m G} * 0.01, X_{i P}=X_{m P} * 0.01, i \neq m, k .
$$

3) Calculate approximate values for the key isotope $\left({ }^{238} \mathrm{U}\right)$ using the isotope normalizations in Eqs. (3ae). The equation for $X_{k P}$ is given below, and analogous equations hold for the other external stream assays:

$$
X_{k P}=1-\sum_{\substack{i=1, i \neq k}}^{I} X_{i P}
$$


4) Calculate stage numbers from Eqs. (25a-d) using key assays from Eq. (31) and the matched assays supplied as input.

5) Calculate the minor isotope assays for top product, side product, and waste streams from Eqs. (2b), (23), and (28). Note that the use of Eqs. (25a-d) in step 4 precludes use of these equations for the key and matched isotopes because these equations are not independent (see APPENDIX C). Because these equations are nonlinear, we adopt a simple iterative scheme that uses a secant method update:

a) Assign initial estimates calculated in step 2, which provides $X_{i P}^{0}$.

b) Calculate updates $X_{i G}$ from Eq. (28) and $X_{i W}$ from Eq. (2b).

c) Calculate from Eq. (23a) using the latest updates for all quantities:

$C_{0}=W X_{i W} \alpha_{i}^{*}+P X_{i P}^{0}\left(\alpha_{i}^{*}\right)^{-N_{P}}+G X_{i G}\left(\alpha_{i}^{*}\right)^{-N_{G}}+F X_{i F}\left(\alpha_{i}^{*}\right)^{-N_{F}}+E X_{i E}\left(\alpha_{i}^{*}\right)^{-N_{E}}$

d) If $\left|C_{0}\right|<\epsilon$, terminate iteration.

e) Modify product assay $X_{i P}^{1}=\gamma X_{i P}^{0}$ and repeat steps b and c to obtain $C_{1}$. The multiplier is either 0.99 or 1.01 , depending on the value of $C_{0}$.

f) If $\left|C_{1}\right|<\epsilon$, terminate iteration.

g) Calculate a new product assay using the secant method:

$$
X_{i P}^{N e w}=X_{i P}^{1}+\frac{C_{1}\left(x_{i P}^{0}-x_{i P}^{1}\right)}{\left(C_{1}-C_{0}\right)}
$$

h) The new update replaces the worst of the previous estimates (i.e., $\max \left\{\left|C_{0}\right|,\left|C_{1}\right|\right\}$ ), and steps $\mathrm{f}-\mathrm{h}$ are repeated until convergence is achieved.

In practice, 3-20 iterations have usually been sufficient to provide convergence for $\epsilon=10^{-12}$. This is true even though the minor isotope initialization in Eq. (30) may be quite coarse, as in the case of reprocessed uranium where the ${ }^{236} \mathrm{U}$ content may be as large as the ${ }^{235} \mathrm{U}$ value.

Note that steps 2 and 3 of the larger process involve approximations and must be iteratively refined as well. This outer iteration usually only requires a single repeat.

This solution approach has been demonstrated in practice to be quite robust, allowing a solution to be obtained for virtually any input values, provided that all input parameters in Table 7 are supplied. For this reason, we employ this same solution procedure in the next section even when the user-supplied inputs vary from those specified in Table 7.

\subsubsection{Generalized Solution}

We wish to allow more flexible input options (i.e., substitutions of other variables for those required by the Von Halle solution in Section 5.1.1). Table 7 requires that all feed assays are known and assays for the matched component are known in all external streams. It may be possible that a solution could still be 
obtained if other assays (such as those for minor isotopes) or flows are substituted for those required by the Von Halle solution. It may even be possible to substitute other variables such as stage numbers if the separation factor is not known. Even though we have conducted extensive testing of the code, minimum requirements for input variables have not been conclusively addressed. Consequently, the user should be careful about deviating too strongly from the input recommendations in Section 5.1.1.

To begin, we define two sets of variables:

$$
\begin{gathered}
Y=\{y \mid y \text { is an input variable supplied by the user }\} \\
Y_{V H}=\{y \mid y \text { is an input variable required by the Von Halle formulation }\} .
\end{gathered}
$$

Note that elements of $Y_{V H}$ are listed in Table 7 at the beginning of the previous section. For each input variable, we consider the following situations:

$$
\begin{aligned}
& y \in Y \cap y \notin Y_{V H} \Rightarrow y \in G, \\
& y \notin Y \cap y \in Y_{V H} \Rightarrow y \in Z .
\end{aligned}
$$

Thus, the set $G$ contains all input variables that are supplied by the user, but are not required by the Von Halle formulation; these variables are arranged in a vector $\hat{\boldsymbol{g}}=\left(\hat{g}_{1}, \hat{g}_{2}, \ldots \hat{g}_{I_{g}}\right)^{T}$, where $\hat{g}_{i} \in G$. Conversely, the set $\mathrm{Z}$ contains all input variables that are required by the Von Halle formulation, but are not initially supplied, and must be computed. The variables in Eq. (34b) are arranged in a vector $\boldsymbol{Z}=\left(z_{1}, z_{2}, \ldots z_{I_{z}}\right)^{T}$, where $z_{i} \in Z$. (Note that some variables may not be in either set; that is, they are given and required by the Von Halle algorithm, or else not given and not required.)

A new algorithm is devised that uses an initial estimate of $\mathbf{z}$ and runs the Von Halle algorithm; output from this calculation include computed values $g_{i} \in G$ that are arranged in the vector $\boldsymbol{g}=\left(g_{1}, g_{2}, \ldots g_{I_{g}}\right)^{T}$ which is then compared to the stated input $\hat{\boldsymbol{g}}$. The estimate of $\boldsymbol{z}$ is refined until the computed $\boldsymbol{g}$ closely matches the given input $\hat{\boldsymbol{g}}$. This entire process is summarized by the optimization problem: determine the optimal vector $\boldsymbol{g}^{*}$, where

$$
\boldsymbol{g}^{*}=\underset{\mathbf{z}}{\operatorname{argmin}}\|\boldsymbol{g}-\hat{\boldsymbol{g}}\|^{2}=\underset{\mathbf{z}}{\operatorname{argmin}} \sum_{i}\left[g_{i}(\mathbf{z})-\hat{g}_{i}\right]^{2} .
$$

The optimization can be performed by any number of procedures. In practice we use a combination of steepest descent, Broyden-Fletcher-Goldfarb-Shanno (BFGS), and Gauss-Newton algorithms, together with line search as proposed by Fletcher [14]. This approach has been used successfully in a number of previous applications, including the MSTAR'12 code.

\subsection{AUXILIARY CALCULATIONS}

Once a solution from Section 5.1 has been obtained, the entire cascade is completely defined. However, additional output may be useful and can be calculated in a straightforward manner. This includes stagewise quantities and perhaps modifications that may assist the analyst such as integer stages. 


\subsubsection{Stagewise Quantities}

After calculating the overall cascade variables in

Table 5, we now wish to calculate stagewise flows and assays. First, we note from Figure 33 that all external streams other than the waste stream leave from the top of a designated stage. Furthermore, the stage variables $N_{P}, N_{G}, N_{F}$, and $N_{E}$ are not constrained to be integers. The governing equations cast them as real variables, and contrary to a real enrichment plant, they often are not integers. Thus, when fractional stages are present, interpretation of stagewise quantities may be somewhat confusing.

Because the top product is usually the most important output quantity, we start with stage $N_{P}$ and progress downward through each region of the cascade. As previously observed in Section 4.4.1, $\Lambda_{i, N_{P}+1}^{\prime \prime}$ $=0$, and from Eq. (12a) we calculate new stage values progressively downward with the equalities:

$$
\Lambda_{i, n}^{\prime \prime}=\frac{\tau_{\text {in }}+\Lambda_{i, n+1}^{\prime \prime}}{\alpha_{i}^{*}},
$$

where in the topmost section $\tau_{\text {in }}=P X_{i P}$. This is followed by calculating the upflowing assays from Eq. (13) and the stagewise feed, product, and tails flows and assays from definitions and Eqs. (4a-c):

$$
\begin{gathered}
L_{n}=\sum_{i} \Lambda_{i, n}, \quad L_{n}^{\prime}=\sum_{i} \Lambda_{i, n}^{\prime}, \quad L_{n}^{\prime \prime}=\sum_{i} \Lambda_{i, n}^{\prime \prime}, \\
x_{i n}=\frac{\Lambda_{i, n}}{L_{n}}, \quad y_{i n}=\frac{\Lambda_{i, n}^{\prime}}{L_{n}^{\prime}}, \quad z_{i n}=\frac{\Lambda_{i, n}^{\prime \prime}}{L_{n}^{\prime \prime}} .
\end{gathered}
$$

These equations all remain true within a distinct region. When a region boundary is encountered, then a partial stage is likely to result, with the downflowing stream calculated by Eq. (14) instead of Eq. (36a). The upflowing stream is still calculated by Eq. (13).

The four regions are designated as follows:

$\begin{array}{cc}\text { Region } & \text { Stages } \\ \text { Upper enriching section } & N_{P}, N_{P-1}, \ldots, N_{G+1} \\ \text { Lower enriching section } & N_{G}, N_{G-1}, \ldots, N_{F+1} \\ \text { Upper stripping section } & N_{F}, N_{F-1}, \ldots, N_{E+1} \\ \text { Lower stripping section } & N_{E}, N_{E-1}, \ldots, N_{1}\end{array}$

If one of the side streams is not present, then the lower and upper sections are combined to form a single enriching or stripping section. We will always have a value for $N_{P}$ and $N_{F}$ (and $N_{W}$, which is always 1 ).

\subsubsection{Integer Stages}

Real enrichment facilities have integer numbers of stages. Although the cascade equations were derived with this in mind, and the subtle implication that the stage numbers were integers, in fact the solutions to the governing equations (as described in the Section 5.1) clearly allow for the stage numbers to be real numbers. However, to make the calculation more consistent with real operations and simplify the output 
somewhat, we may wish to constrain the problem to have integer numbers of stages. In this case, we will have to either relax the values of other variables or allow the governing equations to be satisfied only approximately. The latter case is not particularly desirable because it will likely result in compromise of mass balance or isotopic normalization, which itself is confusing. Hence, we choose to relax some of the specified variables, assays in particular. From Eqs. (25a-d), the stage numbers depend directly on the matched and key component assays of external streams. Any other dependence is minimal and indirect. Hence, these assays are principal choices for relaxation, unless they are already unspecified.

After obtaining a solution without the constraint of integer stages, we use this result as an initial estimate for modeling integer stages. We then conduct a second calculation (completely automated internally) according to the following steps:

1) Round each of the stage numbers $N_{P}, N_{G}, N_{F}$, and $N_{E}$ to the nearest integer.

2) For each stage number, include it in the set $Y$ described in Eq. (33a). If a side stream is missing, it is not included in the analysis.

3) For each stage number, choose an assay at that stage to remove from set $Y$.

4) Rerun the generalized model. The assays whose values have been relaxed will vary somewhat from their original values, but all balance equations will be satisfied exactly.

In some cases, the automated rounding in step 1 may be unsatisfactory, and the user may prefer different stage numbers. In this case, the user can specify directly the desired stage numbers, as illustrated in Figure 24.

\subsection{CASES WITHOUT BOTH SIDE STREAMS}

The development and solution procedure in Sections 5.1 and 5.2 are made assuming that both side feed and side product streams are present. However, many cases may lack one or both of these quantities. The solution procedures outlined in Section 5.2 still apply, with appropriate modifications as described in this section. In addition, the auxiliary calculations from Section 5.2 will also apply in a straightforward manner to these cases. We recall from

Table 5 and Table 6 that when all side streams are present, the total number of variables is $10+5 I$, and the total number of equations is $5+3 I$. We also recall that the required number of inputs from Table 7 is the difference between these two amounts, or $5+2 I$. Note that when counting numbers of isotopes, Table 5 considers all of them so that each isotopic variable includes $I$ values. However, when considering isotopic variables in Table 6 and Table 7, they have fewer than $I$ values. Table 8 summarizes the numbers of variables, equations, and inputs for each of the cases of varying side streams.

Table 8. Number of variables, equations, and inputs.

\begin{tabular}{|l|c|c|c|}
\hline \multicolumn{1}{|c|}{ Description } & Variables & Equations & Inputs \\
\hline Base case-all external streams & $10+5 I$ & $5+3 I$ & $5+2 I$ \\
\hline No side feed & $8+4 I$ & $3+3 I$ & $5+I$ \\
\hline No side product & $8+4 I$ & $5+2 I$ & $3+2 I$ \\
\hline No side streams & $6+3 I$ & $3+2 I$ & $3+I$ \\
\hline
\end{tabular}




\subsubsection{No Side Feed}

In this case, the feed mass flow rate $E$ is zero, and the assays $X_{i E}$ and stage number $N_{E}$ are irrelevant (see Table 5). Hence the problem has $2+I$ fewer variables, resulting in $8+4 I$ remaining variables. The number of equations (cf. Table 6) is reduced by 2 because Eq. (3d) is no longer needed, and Eq. (25e) replaces Eqs. $(25 \mathrm{c}, \mathrm{d})$. With only $3+3 I$ equations, the number of required inputs would be $(8+4 I)-$ $(3+3 I)=5+I$. By eliminating side feed flow rate $E$ and assays $X_{i E}$ from Table 7 , we recognize that there are exactly $5+I$ remaining input variables. These numbers are summarized in the second row of Table 8 .

The solution procedures in Sections 5.1.1 and 5.1.2 can now be applied to the reduced set of equations with virtually no further modifications. Stagewise quantities or integer stages can also be incorporated with little difficulty.

\subsubsection{No Side Product}

This case is similar to the absence of side feed but slightly more complicated because Eq. (28) is no longer applicable or necessary. The flow rate $G$ is zero and side product assays $X_{i G}$ and stage number $N_{G}$ are irrelevant. As in the case of no side feed, there are $2+I$ fewer variables, leaving $8+4 I$ remaining. The number of equations is reduced by elimination of Eq. (3b), Eq. (28), and replacing Eqs. (25a,b) with Eq. (25f). This reduces the number of equations by $2+(I-2)=I$, so that $5+2 I$ equations remain. Thus, we expect that the number of required inputs would be $(8+4 I)-(5+2 I)=3+2 I$. From Table 7 , this is exactly the number of inputs remaining after the elimination of the matched assay $X_{m G}$ and the side product flow rate $G$.

As in the case of side feed, the solution procedures from Sections 5.1.1 and 5.1.2 can be implemented with little difficulty.

\subsubsection{No Side Streams}

This case combines those of Sections 5.3.1 and 5.3.2. Both side stream flow rates are zero, and the corresponding assays and stage numbers are irrelevant. There are $4+2 I$ fewer variables, resulting in 6 $+3 I$ remaining. The number of equations is reduced by $2+I$, resulting in $3+2 I$ remaining. Thus, the number of inputs would be $(6+3 I)-(3+2 I)=3+I$. This number is verified in Table 7 by removing the assays $X_{i E}$ and $X_{m G}$, together with the flow rates $E$ and $G$, which leaves exactly $3+I$ inputs remaining. 


\subsection{INCORPORATION OF MACHINE FLOW}

In some cases, it may be helpful to incorporate feed flow rates for individual centrifuge machines and to estimate the numbers of machines per stage. The latter can be determined from the stage flows, although this issue is complicated somewhat by the possibility of fractional stages. If the total number of machines is known, then they must be apportioned among all the stages according to the flow in each stage. If the feed flow rate into each machine is also known, then this provides an estimate for the throughput for the entire cascade. If the cascade throughput and machine flow are known, then the number of machines can be estimated. These quantities are related by the simple equations:

$$
L=m M, \quad M_{n}=\frac{L_{n}}{m^{\prime}}
$$

where $L$ is the total cascade flow rate, $M$ is the total number of machines, and $m$ is the feed flow rate into each machine. The total cascade flow rate comprises all the feed flow in every stage, and is obtained by summing the overall flows in each region of the cascade:

$$
L=L^{(P)}+L^{(G)}+L^{(F)}+L^{(W)} .
$$

The regional flow rates are derived in APPENDIX D by summing all the stage flows within each region and are given by Eqs. (D.6), (D.9), (D.10), and (D.14). This calculation is nontrivial because of the possibility of fractional stages. Thus, while each of the numbers in Eqs. (37) and (38) is treated as a real number, practicality suggests that the number of machines in a fractional stage is a spurious value; hence, when stagewise output occurs, we do not include numbers of machines for fractional stages. Because there is a possibility of conflicting input values, the following hierarchy is honored in determining output:

1) If neither $M$ nor $m$ is specified on input, then no machine output is included.

2) If either $M$ or $m$ is specified, then the other is calculated from Eq. (37) to be consistent with the specified external cascade flows $(W, E, F, G, P)$; if no external flows are specified, then default values are used $(F=1, E=G=0)$ and a default is used for the unspecified value of $M$ or $m(M=1,000$, $\left.m=280 \mathrm{mg} / \mathrm{s} \mathrm{UF}_{6}\right)$.

3) If both $M$ and $m$ are given, then the external cascade flows $(W, E, F, G, P)$ are scaled from their input values (specified or default) to be consistent with the stagewise and total cascade flows in Eq. (37). Thus, if $L^{U}$ is the value of total flow calculated from Eq. (D.15) using the standard input values for external flows, and $L$ is calculated from Eq. (37a) from the machine input, then a scaling constant is determined as and every flow $\left(W, E, F, G, P, L_{n}, L_{n}^{\prime}, L_{n}^{\prime \prime}\right)$ is multiplied by this constant.

$$
\mu=\frac{L}{L^{U}}
$$




\section{SUMMARY AND CONCLUSIONS}

The MSTAR2019 model has been described in excruciating detail, and its use for a variety of problems has been demonstrated and explained. The GUI has been developed to facilitate use of the model by users who may not be familiar with the mathematical details. Extensive descriptions of the various GUI screens were provided to illustrate a variety of options available to the user. Several enrichment scenarios were demonstrated in sample problems, including most imaginable deviations from declared operations. These can be computed almost instantly, and the results can be quickly downloaded to CSV files for additional processing.

Mathematical derivations have been given for the original equations of Von Halle on which the original MSTAR code was based. These have been adapted somewhat to enhance understandability, and a small correction was implemented. The equations contribute naturally to explanations of the constraints on input variables and to limitations of the algorithm. The use of side streams and the more flexible input options presents a number of advantages in analysis but does complicate the mathematical description of the problem and the implementation by the user. Hence, more extensive quality analysis has been required, and considerably more guidance for the user has been provided.

The original algorithm of Von Halle posed strict input requirements, including specification of exact feed assays and output (i.e., waste and product) assays of ${ }^{235} \mathrm{U}$. The current code allows flexibility in that minor isotopes may be substituted for ${ }^{235} U$ under certain constraints. In addition, the MSTAR2019 code allows the user to impose integer numbers of stages or to specify the stage at which various external streams occur (rather than the assay at that stage). These additional features contribute to the complexities of the underlying algorithm, but most of this is not obvious to the user.

Many diagnostics have been included to assist the user. Most of these address deficiencies in usersupplied inputs, but some identify problems with the user-defined scenarios. This latter category includes problems with solutions that are not physically possible, such as a waste stream with a ${ }^{235} \mathrm{U}$ assay that is higher than the feed or product assays. Every effort has been made to identify scenarios that generally will not or cannot work, either because of poor problem definition or nonphysical behavior. However, the creativity of users may yet identify other difficulties that have not been investigated or anticipated by the developers. For such adverse experiences, we apologize in advance and eagerly solicit user input for improvements in any subsequent versions of the code. 


\section{REFERENCES}

1. A. de la Garza, G. A. Garrett, and J. E. Murphy, "Multicomponent isotope separation in cascades," Chem. Engr. Sci. 15, 188-209 (1961).

2. A. de la Garza, "A generalization of the matched abundance-ratio cascade for multicomponent isotope separation," Chem. Engr. Sci. 18, 73-82 (1963).

3. M. Benedict, T. H. Pigford, and H. W. Levi, Nuclear Chemical Engineering (2nd Ed.), McGraw-Hill, New York (1981).

4. E. Von Halle, "Multicomponent isotope separation in matched abundance ratio cascades composed of stages with large separation factors," pp. 325-56 in K. G. Roesner, Ed., Proceedings of the 1st Workshop on Separation Phenomena in Liquids and Gases, Institut für Mechanik, Darmstadt, FRG (1987).

5. E. Von Halle, unpublished notes, 2007, in possession of author.

6. D. F. Starr, MSTAR-IAEA Enrichment Code User Guide, K/NSP-386, National Security Programs Office, Oak Ridge K-25 Site (June 1996).

7. S. Blumkin and E. Von Halle, The Behavior of the Minor Uranium Isotopes in Separation Cascades, Part IV, K-1839 Part 4, Union Carbide Corporation Nuclear Division, Oak Ridge Gaseous Diffusion Plant (Dec. 18, 1974).

8. H. G. Wood, "Effects of separation processes on minor uranium isotopes in enrichment cascades," Sci. and Glob. Sec. 16, 26-36 (2008).

9. C. F. Weber, MSTAR'12: A Generalized Code for an Ideal, Matched-Abundance-Ratio Enrichment Cascade, Sponsor Report to IAEA (2012).

10. C. F. Weber, "Generalized modeling of enrichment cascades that include minor isotopes," presented at the 53rd Annual Meeting of the Institute for Nuclear Materials Management, Palm Desert, CA (2012).

11. W. G. May, "Separation parameters of gas centrifuges," AIChE Symposium Series 73, Number 169, 30-38 (1977).

12. K. Cohen, The Theory of Isotope Separation as Applied to the Large Scale Production of U-235, National Nuclear Energy Series, Manhattan Project Technical Section, McGraw-Hill, New York (1951).

13. H. G. M. Harink-Snijders, "Solving the diffusion equation for multi-isotope mixtures," Proceedings of the Third Workshop on Separation Phenomena in Liquids and Gases, Charlottesville, VA, Aug. 16-20, 1992.

14. R. Fletcher, Practical Methods of Optimization, 2nd Ed., John Wiley and Sons, New York (1987). 



\section{APPENDIX A. DERIVATION OF EQ. (12)}

From Eq. (11), denote the quantity in brackets by $\sigma$ and evaluate the equation at the (nonexistant) stage above the top stage of the cascade. Since there is no downflowing stream into stage $N_{P}$, the value of $\Lambda_{i, N_{P}+1}^{\prime \prime}$ is zero. Successive recursive applications of Eq. (11) yield:

$$
\begin{gathered}
\Lambda_{i, N_{P}+1}^{\prime \prime}=0=\sigma \alpha_{i} \Lambda_{i, N_{P}}^{\prime \prime}-\tau_{i}=\sigma \alpha_{i}\left(\sigma \alpha_{i} \Lambda_{i, N_{P}-1}^{\prime \prime}-\tau_{i}\right)-\tau_{i}=\left(\sigma \alpha_{i}\right)^{2} \Lambda_{i, N_{P}-1}^{\prime \prime}-\tau_{i}\left(1+\sigma \alpha_{i}\right) \\
=\left(\sigma \alpha_{i}\right)^{2}\left(\sigma \alpha_{i} \Lambda_{i, N_{P}-2}^{\prime \prime}-\tau_{i}\right)-\tau_{i}\left(1+\sigma \alpha_{i}\right)=\left(\sigma \alpha_{i}\right)^{3} \Lambda_{i, N_{P}-2}^{\prime \prime}-\tau_{i}\left[1+\sigma \alpha_{i}+\left(\sigma \alpha_{i}\right)^{2}\right] \\
=\ldots=\left(\sigma \alpha_{i}\right)^{N_{P}+1-n} \Lambda_{i, n}^{\prime \prime}-\tau_{i}\left[1+\sigma \alpha_{i}+\left(\sigma \alpha_{i}\right)^{2} \ldots+\left(\sigma \alpha_{i}\right)^{N_{P}-n}\right] \\
=\left(\sigma \alpha_{i}\right)^{N_{P}+1-n} \Lambda_{i, n}^{\prime \prime}-\tau_{i} \frac{1-\left(\sigma \alpha_{i}\right)^{N_{P}-n+1}}{1-\sigma \alpha_{i}}
\end{gathered}
$$

which can be rearranged to give

$$
\Lambda_{i, n}^{\prime \prime}=\tau_{i} \frac{1-\left(\sigma \alpha_{i}\right)^{n-N_{P}-1}}{\sigma \alpha_{i}-1}
$$

The abundance ratio for the matched component of downflowing stream in stage $n$ can be represented using Eqs. (7a) and (A.1) as

$$
R_{m, n}^{\prime \prime}=\frac{\Lambda_{m, n}^{\prime \prime}}{\Lambda_{k, n}^{\prime \prime}}=\frac{\tau_{m} \frac{1-\left(\sigma \alpha_{m}\right)^{n-N_{p}-1}}{\sigma \alpha_{m}-1}}{\tau_{k} \frac{1-\left(\sigma \alpha_{k}\right)^{n-N_{p}-1}}{\sigma \alpha_{k}-1}}
$$

Recalling that $R_{m, n+1}^{\prime \prime}=\beta_{m} R_{m, n}^{\prime \prime}$ and that $\alpha_{k}=1$ and $\alpha_{m}=\beta_{m}^{2}$, we can write

$$
\frac{R_{m, n+1}^{\prime \prime}}{R_{m, n}^{\prime \prime}}=\beta_{m}=\frac{1-\left(\sigma \beta_{m}^{2}\right)^{n-N_{p}}}{1-\sigma^{n-N_{p}}} \frac{1-\sigma^{n-N_{p}-1}}{1-\left(\sigma \beta_{m}^{2}\right)^{n-N_{p}-1}}
$$

Substituting the value $\sigma=\beta_{m}^{-1}$ into the right side gives

$$
\frac{1-\beta_{m}^{n-N_{p}}}{1-\beta_{m}^{N_{p}-n}} \times \frac{1-\beta_{m}^{N_{p}+1-n}}{1-\beta_{m}^{n-N_{p}-1}} \times \frac{\beta_{m}^{N_{p}-n} \beta_{m}}{\beta_{m}^{N_{p}+1-n}}=\frac{\beta_{m}^{N_{p}-n}-1}{1-\beta_{m}^{N_{p}-n}} \times \frac{1-\beta_{m}^{N_{p}+1-n}}{\beta_{m}^{N_{p}+1-n}-1} \beta_{m}=\beta_{m}
$$

Thus, the value $\sigma=\beta_{m}^{-1}$ satisfies Eq. (A.2) exactly and is therefore used to obtain Eq. (12) in Section 2.2. 


\section{APPENDIX B. VON HALLE TREATMENT OF SECTION JUNCTION POINTS}

As mentioned in the Section 2.3, it appears from Figure 35 that we should equate the two downflowing streams at the junction, in which case Eqs. $(16 \mathrm{a}-\mathrm{c})$ result. This is contrary to the treatment by Von Halle $[5,6]$, which assumes equality for the two upflowing streams and places the accounting for the external stream with the downflowing streams:

$$
\Lambda_{i, N_{G}}^{\prime(G)}=\Lambda_{i, N_{G}}^{\prime(P)} \quad \text { and } \quad \Lambda_{i, N_{G}}^{\prime \prime}(G)=\Lambda_{i, N_{G}}^{\prime \prime(P)}-G X_{i G} .
$$

This appears to be an error in derivation. He uses similar equations for the junction points of other sections:

$$
\Lambda_{i, N_{F}}^{\prime(F)}=\Lambda_{i, N_{F}}^{\prime(G)} \quad \text { and } \quad \Lambda_{i, N_{F}}^{\prime \prime(G)}+1=\Lambda_{i, N_{F}+1}^{\prime \prime(F)}-F X_{i F}
$$

and

$$
\Lambda_{i, N_{E}}^{\prime}(E)=\Lambda_{i, N_{E}}^{\prime}(F) \quad \text { and } \quad \Lambda_{i, N_{E}}^{\prime \prime}(F)+1=\Lambda_{i, N_{E}}^{\prime \prime}(E)+1-E X_{i E}
$$

Using Eqs. (B.1a-c) instead of Eqs. (16a-c) results in the following cascade equation, which differs slightly from Eq. (23):

$$
P X_{i P}\left[1-\left(\alpha_{i}^{*}\right)^{-N_{P}-1}\right]+G X_{i G}\left[1-\left(\alpha_{i}^{*}\right)^{-N_{G}}\right]+F X_{i F}\left[1-\left(\alpha_{i}^{*}\right)^{-N_{F}}\right]+E X_{i E}\left[1-\left(\alpha_{i}^{*}\right)^{-N_{E}}\right]=0
$$

Remembering nomenclature changes, it is instructive to compare Eqs. (23) and (B.2) with Von Halle's original derivation without side streams (i.e., $G=E=0$ ). The equations are developed in Ref. [4] and were used in MSTAR'12. A comparison of nomenclature is shown in Table B.1 below. The cascade equations from Ref. [4] can be expressed as

$$
S_{i} P X_{i P}-E_{i} W X_{i W}
$$

where $M$ and $N$ are the numbers of stages above and below the main feed stream (i.e., in the stripping and enriching sections, respectively), and

$$
S_{i} \equiv \frac{\alpha_{i}^{*}-1}{\left(\alpha_{i}^{*}\right)^{M+1}-1} \quad \text { and } \quad E_{i} \equiv \frac{\alpha_{i}^{*}-1}{1-\left(\alpha_{i}^{*}\right)^{-N}}
$$

Substituting Eqs. (B.4) into Eq. (B.3) and simplifying gives

$$
\left[1-\left(\alpha_{i}^{*}\right)^{-N}\right] P X_{i P}-\left[\left(\alpha_{i}^{*}\right)^{M+1}-1\right] W X_{i W}=0 .
$$


The analogues in MSTAR2019 for the values of $M$ and $N$ are given in Table B.1. Recognizing that the isotope balance $-W X_{i W}=P X_{i P}+F X_{i F}$ remains true for either version of MSTAR, and substituting the values from Table B.1 into Eq. (B.5) gives

$$
\left[1-\left(\alpha_{i}^{*}\right)^{N_{F}-N_{P}}\right] P X_{i P}+\left[\left(\alpha_{i}^{*}\right)^{N_{F}+1}-1\right]\left(P X_{i P}+F X_{i F}\right)=0
$$

Multiplying through by $\left(\alpha_{i}^{*}\right)^{-N_{F}}$ and rearranging gives

$$
\left[1-\left(\alpha_{i}^{*}\right)^{-N_{P}-1}\right] P X_{i P}+\left[1-\left(\alpha_{i}^{*}\right)^{-N_{F}-1}\right] F X_{i F}=0
$$

which matches Eq. (23) but not Eq. (B.2). We thus conclude that Eq. (23) is the correct form for the cascade equation.

Table B.1. Stage nomenclature in original MSTAR'12 and MSTAR2019.

\begin{tabular}{|l|c|c|}
\hline \multicolumn{1}{|c|}{ Quantity } & MSTAR'12 & MSTAR2019 \\
\hline Total stages & $N+M$ & $N_{P}$ \\
\hline Enriching stages & $N$ & $N_{P}-N_{F}$ \\
\hline Stripping stages & $M$ & $N_{F}$ \\
\hline
\end{tabular}




\section{APPENDIX C. DEPENDENCES OF GOVERNING EQUATIONS}

A number of equations have been derived in Section 2, but we now observe that not all of them are independent. Thus, when devising a methodology to calculate unknown process parameters, we need to ensure that we have sufficient information to actually achieve a solution. It is fairly straightforward to see that summing Eq. (2b) over the number of isotopes, and applying the normalizations in Eq. (3), yields the overall flow balance given in Eq. (1). In the remainder of this appendix, we show the dependences between Eqs. (25a-d) and other equations.

We note that the stage number equations in Section 2.6 refer only to the matched component $m$ and the key component $k$; hence, Eqs. (25a-d) cannot be generalized to include any other components. However, we also note that Eqs. (23) and (28) are true for any components, and there is the distinct possibility of some redundancy (i.e., linear dependence) of Eqs. (23) and (28) for the cases of $i=m$ or $k$.

Note first that there are several common variables appearing in Eq. (25a) and Eq. (28). We begin by evaluating Eq. (28) for $i=m$ and $i=k$ and taking the ratio. The summation in the denominator is canceled out to leave

$$
\frac{X_{m G}}{X_{k G}}=\frac{X_{m P} \alpha_{m}^{*}\left[\frac{1-\left(\alpha_{m}^{*}\right)^{N_{G}-N_{P}-1}}{\alpha_{m}^{*}-1}\right]}{X_{k P} \alpha_{k}^{*}\left[\frac{1-\left(\alpha_{k}^{*}\right)^{N_{G}-N_{P}-1}}{\alpha_{k}^{*}-1}\right]}
$$

From Eqs. (1) and (9), we have

$$
\alpha_{m}^{*}=\frac{\alpha_{m}}{\beta_{m}}=\beta_{m} \quad \text { and } \quad \alpha_{k}^{*}=\frac{\alpha_{k}}{\beta_{m}}=\frac{1}{\beta_{m}},
$$

this becomes

$$
\begin{aligned}
& \frac{X_{m G}}{X_{k G}} \\
& \left.=\frac{X_{m P} \beta_{m}\left[\frac{1-\left(\beta_{m}\right)^{N_{G}-N_{P}-1}}{\beta_{m}-1}\right]}{X_{k P}\left(\frac{1}{\beta_{m}}\right)\left[\frac{1-\left(\beta_{m}\right)^{-N_{G}+N_{P}+1}}{\left(1 / \beta_{m}\right)-1}\right]}=\frac{X_{m P}\left[\frac{\beta_{m}-\left(\beta_{m}\right)^{N_{G}-N_{P}}}{\beta_{m}-1}\right]}{X_{k P}\left[\frac{1-\left(\beta_{m}\right)^{-N_{G}+N_{P}+1}}{1-\beta_{m}}\right]}=\frac{X_{m P}\left[\left(\beta_{m}\right)^{N_{G}-N_{P}}\right.}{X_{k P}\left[1-\left(\beta_{m}\right)^{-N_{G}}\right.} \text { (C.1 }\right) \\
& \beta_{m}^{N_{P}-N_{G}} \text {, }
\end{aligned}
$$

which is identical to Eq. (25a). Thus, Eq. (25a) is not independent of Eq. (28) with $i=m$ and $k$. There are only two independent equations.

Next, we examine dependencies of Eqs. (25a-d) with the cascade equations, Eq. (23). Note that Eq. (25a) can be arranged to get 


$$
\frac{X_{m P}}{X_{k P}} \beta_{m}^{-N_{P}}=\frac{X_{m G}}{X_{k G}} \beta_{m}^{-N_{G}}
$$

Similar developments for Eqs. (25b-d) result in the string of equalities:

$$
\frac{X_{m P}}{X_{k P}} \beta_{m}^{-N_{P}}=\frac{X_{m G}}{X_{k G}} \beta_{m}^{-N_{G}}=\frac{X_{m F}}{X_{k F}} \beta_{m}^{-N_{F}}=\frac{X_{m E}}{X_{k E}} \beta_{m}^{-N_{E}}=\frac{X_{m W}}{X_{k W}} \beta_{m}=\xi .
$$

Each of these quantities must be a constant because it cannot depend on any of the other assays or stage number variables; we denote the constant by $\xi$. Evaluating Eq. (23a) for the matched isotope $(i=m)$, and substituting in for the various terms in Eq. (C.2b) gives

$$
W X_{k W} \xi+P X_{k P} \xi+G X_{k G} \xi+F X_{k F} \xi+E X_{k E} \xi=0 \text {. }
$$

Thus, Eq. (23a) for the matched component $i=m$ is equivalent to the overall mass balance of the key component (i.e., Eq. [2b]) with $i=k$. The reverse is also true. Evaluating Eq. (23a) with $i=k$ and recalling that for the key component $\alpha_{k}^{*}=1 / \beta_{j}$, we have

$$
W X_{k W} \frac{1}{\beta_{m}}+P X_{k P} \beta_{m}^{N_{P}}+G X_{k G} \beta_{m}^{N_{G}}+F X_{k F} \beta_{m}^{N_{F}}+E X_{k E} \beta_{m}^{N_{E}}=0 .
$$

Substituting in the various terms from Eq. (C.2b) gives

$$
W X_{m W} / \xi+P X_{m P} / \xi+G X_{m G} / \xi+F X_{m F} / \xi+E X_{m E} / \xi=0,
$$

which is equivalent Eq. (2b) with $i=m$.

In summary, then, Eqs. (25a-d) and (23) are not independent. If we wish to use Eqs. (25a-d), then we cannot use Eq. (23) or (2b) with $i=m$ and $i=k$, or the product assay Eq. (28) with $i=m$ or $i=k$. 


\section{APPENDIX D. MACHINE FLOWS}

If data are supplied for individual machines, then it is useful to calculate machines per stage, and connect numbers of machines to external flows into (or out of) the cascade. In this appendix, we derive equations for total cascade flow and flow for each stage. The number of machines per stage is then calculated using the total stage flow rate and the flow rate per machine. Heavy use is made of the summation formula for finite power series:

$$
\sum_{n=0}^{N} x^{n}=\frac{1-x^{N+1}}{1-x}
$$

From Eq. (14), we have

$$
\Lambda_{i n}^{\prime \prime}=\left(\alpha_{i}^{*}\right)^{n-j} \Lambda_{i j}^{\prime \prime}-\tau_{i} \frac{1-\left(\alpha_{i}^{*}\right)^{n-j}}{1-\alpha_{i}^{*}},
$$

which holds within each section of the cascade. From Eq. (13) we get the representation for stagewise feed flow:

$$
\Lambda_{i n}=\Lambda_{i n}^{\prime}+\Lambda_{i n}^{\prime \prime}=\left(1+\alpha_{i}^{*}\right) \Lambda_{i n}^{\prime \prime}
$$

We now evaluate Eq. (D.2) for each section of the cascade, then apply Eq. (D.3) to obtain stagewise feed flows and overall cascade flows.

Top Product Section. Since $\Lambda_{i, N_{P}+1}^{\prime \prime}=0$, assigning $j=N_{P}+1$ in Eq. (D.2) yields

$$
\Lambda_{i n}=\left(1+\alpha_{i}^{*}\right) \Lambda_{i n}^{\prime \prime}=-P X_{i P}\left(1+\alpha_{i}^{*}\right) \frac{1-\left(\alpha_{i}^{*}\right)^{n-N_{P}-1}}{1-\alpha_{i}^{*}}
$$

From this we obtain the stagewise flow $L_{n}$ and the total flow in the entire section $L^{(P)}$ :

$$
L_{n}=\sum_{i=1}^{I} \Lambda_{i n}=\sum_{i=1}^{I}-P X_{i P}\left(1+\alpha_{i}^{*}\right) \frac{1-\left(\alpha_{i}^{*}\right)^{n-N_{P}-1}}{1-\alpha_{i}^{*}},
$$

$L^{(P)}$

$$
\begin{aligned}
& \left.=\sum_{n=N_{G}+1}^{N_{P}} L_{n}=\sum_{i=1}^{I}-P X_{i P} \frac{\left(1+\alpha_{i}^{*}\right)}{1-\alpha_{i}^{*}} \sum_{n=N_{G}+1}^{N_{P}}\left[1-\left(\alpha_{i}^{*}\right)^{n-N_{P}-1}\right]=\sum_{i=1}^{I} P X_{i P} \frac{\left(\alpha_{i}^{*}+\right.}{\left(\alpha_{i}^{*}-\right.} \text { (D.6 }\right) \\
& {\left[N_{P}-N_{G}-\frac{1-\left(\alpha_{i}^{*}\right)^{N_{G}-N_{P}}}{\alpha_{i}^{*}-1}\right] .}
\end{aligned}
$$




$$
\text { D-2 }
$$


If $\alpha_{i}^{*}=1$ for some $i$, then the corresponding term in the summation of Eq. (D.6) becomes singular. Multiple applications of L'Hopital's rule simplifies that term to

$$
P X_{i P}\left(N_{P}-N_{G}\right)\left(N_{P}-N_{G}+1\right)
$$

Side Product Section. For the section between side product and feed streams, Eq. (D.2) can be written

$$
\Lambda_{i n}=\left(\alpha_{i}^{*}\right)^{n-j} \Lambda_{i j}-\tau_{i}\left(1+\alpha_{i}^{*}\right) \frac{1-\left(\alpha_{i}^{*}\right)^{n-j}}{1-\alpha_{i}^{*}} .
$$

From this we again get the stagewise flows by summing over all isotopes

$$
L_{n}=\sum_{i=1}^{I} \Lambda_{i n}=\sum_{i=1}^{I}\left[\left(\alpha_{i}^{*}\right)^{n-j} \Lambda_{i j}-\tau_{i}\left(1+\alpha_{i}^{*}\right) \frac{1-\left(\alpha_{i}^{*}\right)^{n-j}}{1-\alpha_{i}^{*}}\right]
$$

and the total section flow by summing over all stages

$$
\begin{aligned}
L^{(G)}= & \sum_{n=N_{F}+1}^{N_{G}} L_{n}=\sum_{i=1}^{I} \Lambda_{i, N_{G}}+1 \frac{1-\left(\alpha_{i}^{*}\right)^{N_{F}-N_{G}}}{\alpha_{i}^{*}-1} \\
& +\sum_{i=1}^{I}\left\{\left(P X_{i P}+G X_{i G}\right) \frac{\left(\alpha_{i}^{*}+1\right)}{\left(\alpha_{i}^{*}-1\right)}\left[N_{G}-N_{F}+\frac{1-\left(\alpha_{i}^{*}\right)^{N_{F}-N_{G}}}{1-\alpha_{i}^{*}}\right]\right\}
\end{aligned}
$$

If $\alpha_{i}^{*}=1$ for any $i$, then the right side of Eq. (D.9) simplifies to

$$
\Lambda_{i, N_{G}+1}\left(N_{G}-N_{F}\right)+\left(P X_{i P}+G X_{i G}\right)\left(N_{G}-N_{F}\right)\left(N_{G}-N_{F}+1\right) .
$$

Main Feed Section. This section is totally analogous to the side product section, so the derivation will not be included. The analogue to Eq. (D.9) is

$$
L^{(F)}=\sum_{i=1}^{I} \Lambda_{i, N_{F}}+1 \frac{1-\left(\alpha_{i}^{*}\right)^{N_{E}-N_{F}}}{\alpha_{i}^{*}-1}+\sum_{i=1}^{I}\left\{( P X _ { i P } + G X _ { i G } + F X _ { i F } ) \frac { ( \alpha _ { i } ^ { * } + 1 ) } { ( \alpha _ { i } ^ { * } - 1 ) } \left[N_{F}-N_{E}+\frac{1-\left(\alpha_{i}^{*}\right)}{1-a}(\mathrm{D} .10)\right.\right.
$$

If $\alpha_{i}^{*}=1$ for some $i$, then the right side of Eq. (D.10) simplifies to

$$
\Lambda_{i, N_{F}+1}\left(N_{F}-N_{E}\right)+\left(P X_{i P}+G X_{i G}+F X_{i F}\right)\left(N_{F}-N_{E}\right)\left(N_{F}-N_{E}+1\right)
$$


Bottom Section. Evaluating Eq. (D.2) for $j=1$ and using Eq. (20a), we have

$$
\Lambda_{i n}=\left(\alpha_{i}^{*}\right)^{n-1} W X_{i W}\left(1+\alpha_{i}^{*}\right)+W X_{i W}\left(1+\alpha_{i}^{*}\right)\left[\frac{1-\left(\alpha_{i}^{*}\right)^{n-1}}{1-\alpha_{i}^{*}}\right]=W X_{i W} \frac{\left(1+\alpha_{i}^{*}\right)}{1-\alpha_{i}^{*}}\left[1-\left(\alpha_{i}^{*}\right)^{n}\right](\mathrm{D} .11
$$

Stagewise flows and total section flows are obtained as before, by summing over isotopes and stages, respectively:

$$
L_{n}=\sum_{i=1}^{I} \Lambda_{i n}=\sum_{i=1}^{I}\left\{W X_{i W} \frac{\left(\alpha_{i}^{*}+1\right)}{\left(\alpha_{i}^{*}-1\right)}\left[\left(\alpha_{i}^{*}\right)^{n}-1\right]\right\}
$$

$$
L^{(W)}=\sum_{n=1}^{N_{E}} L_{n}=\sum_{i=1}^{I}\left\{W X_{i W} \frac{\left(\alpha_{i}^{*}+1\right)}{\left(\alpha_{i}^{*}-1\right)} \sum_{n=1}^{N_{E}}\left[\left(\alpha_{i}^{*}\right)^{n}-1\right]\right\}=\sum_{i=1}^{I}\left\{W X _ { i W } \frac { ( \alpha _ { i } ^ { * } + 1 ) } { ( \alpha _ { i } ^ { * } - 1 ) } \left[\alpha_{i}^{*} \frac{1-\left(\alpha_{i}^{*}\right)^{N_{E}}}{1-\alpha_{i}^{*}}-(\mathrm{D} .13\right.\right.
$$

If $\alpha_{i}^{*}=1$ for some $i$, then the right side of Eq. (D.13) simplifies to

$$
W X_{i W} N_{E}\left(N_{E}+1\right) \text {. }
$$

Total Cascade. The total flow in all stages throughout the cascade is obtained by summing the total flows in each region

$$
L=L^{(P)}+L^{(G)}+L^{(F)}+L^{(W)} .
$$

Note that if there is no side product, then $L^{(G)}=0$ and Eq. (D.6) is replaced by the equation

$$
L^{(P)}=\sum_{i=1}^{I} P X_{i P} \frac{\left(\alpha_{i}^{*}+1\right)}{\left(\alpha_{i}^{*}-1\right)}\left[N_{P}-N_{F}-\frac{1-\left(\alpha_{i}^{*}\right)^{N_{F}-N_{P}}}{\alpha_{i}^{*}-1}\right] .
$$

Similarly, if there is no side feed, then $L^{(F)}=0$ and Eq. (D.13) is replaced by

$$
L^{(W)}=\sum_{i=1}^{I}\left\{W X_{i W} \frac{\left(\alpha_{i}^{*}+1\right)}{\left(\alpha_{i}^{*}-1\right)}\left[\alpha_{i}^{*} \frac{1-\left(\alpha_{i}^{*}\right)^{N_{F}}}{1-\alpha_{i}^{*}}-N_{F}\right]\right\} .
$$




\section{APPENDIX E. SUMMARY OF MODELING EQUATIONS}

Flow balance over entire cascade:

$$
P+G+F+E+W=0 \text {. }
$$

Isotopic mass balance on entire cascade:

$$
P x_{i P}+G x_{i G}+F x_{i F}+E x_{i E}+W x_{i W}=0, \quad i=1, \ldots, I .
$$

Isotopic normalizations at each external entry point:

$$
\sum_{i=1}^{I} X_{i P}=\sum_{i=1}^{I} X_{i G}=\sum_{i=1}^{I} X_{i F}=\sum_{i=1}^{I} X_{i E}=\sum_{i=1}^{I} X_{i W}=1 .
$$

Cascade equations:

$$
W x_{i W} \alpha_{i}^{*}+P x_{i P}\left(\alpha_{i}^{*}\right)^{-N_{p P}}+G x_{i G}\left(\alpha_{i}^{*}\right)^{-N_{g G}}+F x_{i F}\left(\alpha_{i}^{*}\right)^{-N_{F}}+E x_{i E}\left(\alpha_{i}^{*}\right)^{-N_{E}}=0, \quad i=1, \ldots, I .
$$

Product assay equations:

$$
x_{i G}=y_{i N_{G}}=\frac{\Lambda_{i, N_{G}}^{\prime}}{L_{N_{G}}^{\prime}}=\frac{x_{i P} \alpha_{i}^{*}\left[\frac{1-\left(\alpha_{i}^{*}\right)^{N_{G}-N_{P}-1}}{\alpha_{i}^{*}-1}\right]}{\sum_{m} x_{m P} \alpha_{m}^{*}\left[\frac{1-\left(\alpha_{m}^{*}\right)^{N_{G}-N_{P}-1}}{\alpha_{m}^{*}-1}\right]}, \quad i=1, \ldots, I .
$$

Cascade stage number equations:

$$
\begin{aligned}
& N_{P}-N_{G}=\frac{1}{\ln \left(\alpha_{m}^{*}\right)} \ln \left[\frac{x_{m P} / x_{k P}}{x_{m G} / x_{k G}}\right] . \\
& N_{G}-N_{F}=\frac{1}{\ln \left(\alpha_{m}^{*}\right)} \ln \left[\frac{x_{m G} / x_{k G}}{x_{m F} / x_{k F}}\right], \\
& N_{F}-N_{E}=\frac{1}{\ln \left(\alpha_{m}^{*}\right)} \ln \left[\frac{x_{m F} / x_{k F}}{x_{m E} / x_{k E}}\right] . \\
& N_{E}=-1+\frac{1}{\ln \left(\alpha_{m}^{*}\right)} \ln \left[\frac{x_{m E} / x_{k E}}{x_{m W} / x_{k W}}\right] .
\end{aligned}
$$


E-2 
Stage number equations modified for no side feed, replacing Eqs. $(25 \mathrm{c}, \mathrm{d})$ :

$$
N_{F}=-1+\frac{1}{\ln \left(\alpha_{m}^{*}\right)} \ln \left[\frac{x_{m F} / x_{k F}}{x_{m W} / x_{k W}}\right] \text {, }
$$

Stage number equations modified for no side product, replacing Eqs. (25a,b):

$$
N_{P}-N_{F}=\frac{1}{\ln \left(\alpha_{m}^{*}\right)} \ln \left[\frac{x_{m P} / x_{k P}}{x_{m F} / x_{k F}}\right] \text {. }
$$




\section{APPENDIX F. ALTERNATIVE TO ${ }^{238}$ U AS KEY ISOTOPE}

In Sections 3.2.1, 3.2.2, and 3.3.1, a special problem was noted with enriching recycled uranium to very high enrichment levels. This problem is due to the dwindling inventory of ${ }^{238} \mathrm{U}$ and is specially connected to use of this isotope as the key isotope that occurs in the denominator of the abundance ratios in Eq. (6a). From Figure 26, we see that if ${ }^{235} \mathrm{U}$ inventory is to be further increased, it will enrich relative to ${ }^{236} \mathrm{U}$ more than to ${ }^{238} \mathrm{U}$. Hence, we can revise the model by using the product results in Figure 26 as input to a new cascade in which the key isotope is ${ }^{236} \mathrm{U}$ rather than ${ }^{238} \mathrm{U}$.

The separation factor $\alpha_{i}$ is a ratio of abundance ratios, and the key isotope occupies the denominator of each abundance ratio. Hence, if we change the key isotope, we must revise the separation factor as well. The original separation factors are given by Eq. (7), which can be written for ${ }^{238} \mathrm{U}$ as the key isotope:

$$
\alpha_{i}=\frac{R_{i}^{\prime}}{R_{i}^{\prime \prime}}=\frac{y_{i} / y_{238}}{x_{i} / x_{238}}, \quad i=1,2, \ldots, I .
$$

If we define the enrichment factor $\tilde{\alpha}_{i}$ to be the appropriate enrichment factor for ${ }^{236} \mathrm{U}$ as key isotope, then

$$
\tilde{\alpha}_{i}=\frac{y_{i} / y_{236}}{x_{i} / x_{236}}=\frac{y_{i} / y_{238}}{x_{i} / x_{238}} \times \frac{y_{238} / y_{236}}{x_{238} / x_{236}}=\frac{\alpha_{i}}{\alpha_{236}} .
$$

For an enrichment factor of $\alpha=1.5$ and isotopic separation factors determined from Eq. (1), Table F.1 gives the values of $\alpha_{i}$ for ${ }^{238} \mathrm{U}$ as key isotope, and $\tilde{\alpha}_{i}$ determined from Eq. (F.2).

Table F.1. Using ${ }^{236} \mathrm{U}$ as key isotope.

\begin{tabular}{|l|l|l|}
\hline Isotope & \multicolumn{1}{c|}{$\boldsymbol{\alpha}_{\boldsymbol{i}}$} & \multicolumn{1}{c|}{$\tilde{\boldsymbol{\alpha}}_{\boldsymbol{i}}$} \\
\hline $\mathrm{U}-234$ & 1.717071 & 1.310371 \\
\hline $\mathrm{U}-235$ & 1.5 & 1.144714 \\
\hline $\mathrm{U}-236$ & 1.310371 & 1 \\
\hline $\mathrm{U}-238$ & 1 & 0.763143 \\
\hline
\end{tabular}

If we now use the product results from Figure 25 as input to a new cascade in which we enrich to $90 \%$ ${ }^{235} \mathrm{U}$, we obtain results shown in Table F.2. As noted in the table, the waste assays are quite arbitrary and do have a small impact on product values.

Table F.2. Very high enrichment of recycled fuel using ${ }^{236} \mathrm{U}$ as key isotope.

\begin{tabular}{|l|l|l|l|}
\hline Isotope & \multicolumn{1}{|c|}{ Waste $^{*}$} & \multicolumn{1}{|c|}{ Feed } & Product \\
\hline${ }^{234} \mathrm{U}$ & 0.491181 & 2.56755 & 4.64392 \\
\hline${ }^{235} \mathrm{U}$ & 60.0 & 75.0 & 90.0 \\
\hline${ }^{236} \mathrm{U}$ & 36.505 & 20.9297 & 5.35479 \\
\hline${ }^{238} \mathrm{U}$ & 3.00414 & 1.50272 & .0013 \\
\hline
\end{tabular}

* Waste assays are arbitrary 


\section{APPENDIX G. MSTAR INPUT FILE FORMAT}

A sample input file is shown below with a description of each parameter following:

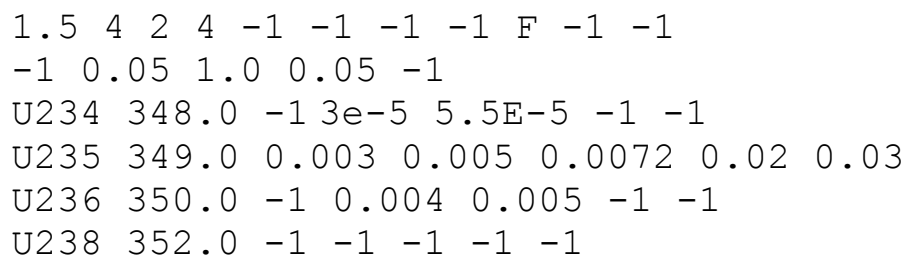

$\underline{\text { Line } 1}$

Separation factor

Number of isotopes

Index of matched isotope (usually ${ }^{235} \mathrm{U}$ )

Index of key isotope (usually ${ }^{238} \mathrm{U}$ )

Stage number of side feed

Stage number of main feed

Stage number of side product

Stage number of top product

Logical flag for integer number of stages $(\mathrm{T}=$ integer, $\mathrm{F}=$ real $)$

Number of machines

Feed flow rate for an individual machine

Line 2

Flow rates in order: W, E, F, G, P (see

Table 5)

Lines 3-6 (for each isotope)

Label

Molecular weight of $\mathrm{UF}_{6}$

Assays in order: $x_{i W}, x_{i E}, x_{i F}, x_{i G}, x_{i P}($ see

Table 5)

Inspection of the input file above indicates that it includes all external streams and supplies all the inputs required in Table 7. A second example is shown below and includes noticeable differences:

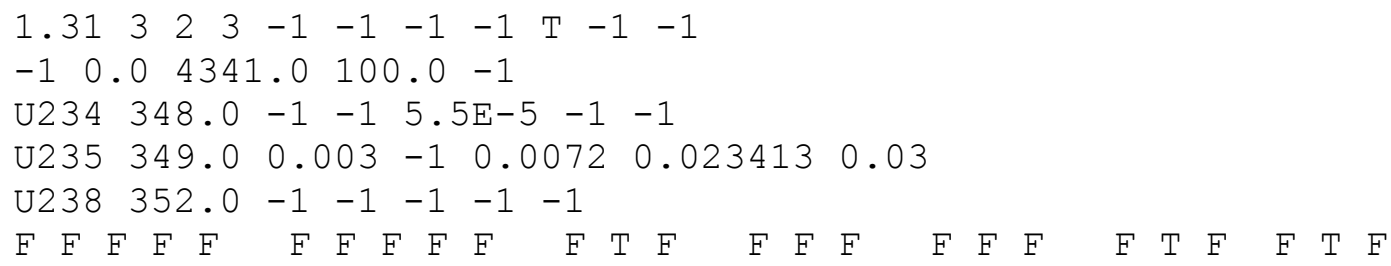

In this file there are only three isotopes, but the "integer stages" flag in the top line is "T." Hence, an additional line is included at the end of the file that specifies "relaxation variables" (i.e., specified variables that will be allowed to stray from their designated values to achieve integer numbers of stages without violating any of the governing equations). The logical flags represent each of the variables in Table 5, and in this case assays of ${ }^{235} \mathrm{U}$ in waste, side product, and product streams are denoted with $\mathrm{T}$, and so become the relaxation variables. 
